\title{
NUMERICAL STUDY OF AEROELASTIC BEHAVIOUR OF A TROPOSKIEN SHAPE VERTICAL AXIS WIND TURBINE
}

\author{
A thesis submitted to \\ the Faculty of Graduate and Postdoctoral Affairs \\ in partial fulfillment of the requirements for the degree \\ Masters of Applied Science
}

by

Amin Fereidooni

The Department of Mechanical and Aerospace Engineering Carleton University

September 2013

(C2013 Amin Fereidooni 


\section{Abstract}

A methodology for aeroelastic analysis of Vertical Axis Wind Turbines (VAWTs) with troposkien geometry is developed. The structural dynamic equations used in this analysis represent the behaviour of a three dimensionally curved beam in terms of generalized force and generalized displacement vectors. This linear formulation takes into account the effect of the Coriolis forces and centrifugal stiffening. It is demonstrated that the mixed form of the equations lends itself well to the application of mixed finite element method.

The structural dynamic equations are coupled with a free vortex based aerodynamic model. The aerodynamic model represents the wake with a cluster of vortex filaments that stretch, rotate and translate freely in the wake of the wind turbine. The unsteady effect of the wake is already taken into consideration when the aerodynamic forces on the blades are evaluated by interpolating the force coefficients from input experimental airfoil data. It is illustrated that in the case of strong vortex-blade interaction at high tip speed ratio, this model can tackle the problem with high accuracy.

The computational aeroelastic framework is utilized to study the aeroelastic behaviour of the 17-meter DOE-Sandia VAWT. It is shown that in the operating range of the wind turbine, the structural vibration has minimum effect on the aerodynamic performance of the turbine. Furthermore, the comparison of the estimated vibratory stress at the root of wind turbine with the experimental data reveals excellent agreement except at the region where dynamic stall plays a crucial role in predicting the aerodynamic forces. 


\section{Acknowledgments}

I would like to extend my gratitude to my supervisors Professor Fred Nitzsche and Professor Edgar Matida for providing an open, friendly and collaborative research environment. I would like to thank Professor Nitzsche for teaching me how to think as an independent researcher. I appreciate Professor Matida for his help any time I had to deal with a challenging situation.

I would also like to thank my friends and former colleagues Anton Matachniouk, Dr. Waad Subber, Dr. Mohammad Khalil, Adam Walker and Hamza Ella for assisting me in my learning process and for making this period of life an enjoyable one. I wish to thank

my current colleagues Hali Barber, Basim Altalua, Doma Suliman and Souhib Aljasim for their help and support.

Furthermore, I thank my family. I am indebted to my parents whose love, support and understanding helped me to pursue graduate studies. Last but not least, I would like to thank my girlfriend, Mahkameh Yaghmaie, for providing me support, encouragement and motivation throughout the course of my studies. 


\section{Contents}

11ntroduction 1

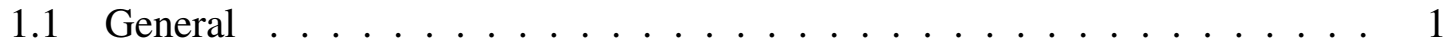

1.2 Literature Survey of the Aeroelastic Analysis of Vertical Axis Wind Turbines 6

1.3 The Proposed Approach for Aeroelastic Analysis of Vertical Axis Wind

Turbines ............................ 9

1.4 Objectives of the Thesis $\ldots \ldots \ldots \ldots \ldots$. . . . . . . . . . 11

1.5 Layout of the Thesis $\ldots \ldots \ldots \ldots \ldots \ldots$

$\begin{array}{lll}2 & \text { Structural Dynamic Analysis } & 13\end{array}$

2.1 Introduction . . . . . . . . . . . . . . . . . . 13

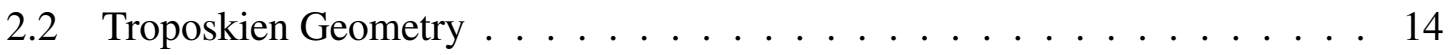

2.3 Structural Analysis . . . . . . . . . . . . . . . . . . 16

$2.3 .1 \quad$ Structural Equilibrium Equations $\ldots \ldots \ldots$

2.3 .2 Mixed Finite Element Formulation . . . . . . . . . . . . . 21

2.4 Dynamic Analysis . . . . . . . . . . . . . . . . . . . 25

2.4 .1 Dynamic Forces . . . . . . . . . . . . . . 25

2.4 .2 Mixed Finite Element Formulation . . . . . . . . . . . . . . 28

2.5 Results andDiscussions . . . . . . . . . . . . . . . . . . 30 
2.5.] Coriolis Force Excluded . . . . . . . . . . . . . . . . . . 31

2.5 .2 Coriolis Force Included . . . . . . . . . . . . . . . 36

2.6 Conclusions . . . . . . . . . . . . . . . . . . 40

$\begin{array}{lll}3 \text { Aerodynamic Analysis } & 41\end{array}$

B. Introduction . . . . . . . . . . . . . . . . . 41

B.2 Eree Vortex Model . . . . . . . . . . . . . . . . . . 42

3.2.1 Coordinate System . . . . . . . . . . . . . . . . 42

3.2 .2 Element Bound Vorticity . . . . . . . . . . . . . . . . . . 43

3.2 .3 Vortex Shedding . . . . . . . . . . . . . . . . 45

B.2.4 Wake Convection . . . . . . . . . . . . . . . . 47

3.2 .5 Remarks . . . . . . . . . . . . . . . . . . 48

3.3 Numerical validation . . . . . . . . . . . . . . . 50

3.4 Convergence Analysis $\ldots \ldots \ldots \ldots \ldots \ldots$

3.4.1 Spatial Convergence . . . . . . . . . . . . . 55

3.4 .2 Temporal Convergence $\ldots \ldots \ldots \ldots \ldots$

3.5 Parallel Implementation . . . . . . . . . . . . . . . 57

5.6 Conclusions . . . . . . . . . . . . . . . . . . . . . . 59

\begin{tabular}{lll}
\hline Aeroelastic Analysis & 61
\end{tabular}

4.1 Introduction . . . . . . . . . . . . . . . . . 61

$4.2 \quad$ Aeroelastic Coupling . . . . . . . . . . . . . . . . . . 62

42. Structurab Deflection . . . . . . . . . . . . . . . . . 62

4.2 .2 Aerodynamic Updates $\ldots \ldots \ldots \ldots \ldots$

4.3 Results andDiscussions . . . . . . . . . . . . . . . . . . . . . . . . . . . . 69

$4.3 .1 \quad$ Aerodynamic Performance $\ldots \ldots \ldots \ldots$ 
4.3 .2 Structural Performance . . . . . . . . . . . . . . . . 73

4.4 Conclusions . . . . . . . . . . . . . . . . . . . 81

5 Conclusions and Recommendations $\quad 82$

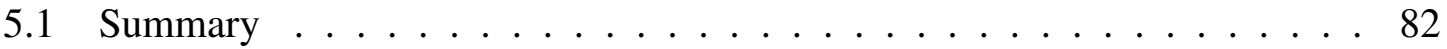

5.2 Conclusion . . . . . . . . . . . . . . . 83

5.3 Recommendations and Future Work $\ldots \ldots \ldots \ldots$. . . . . . . . 84

\begin{tabular}{ll}
\hline Appendices & 86
\end{tabular}

A Appendix A: Aerodynamic Updates due to the Structural Vibration 86

A.1 Coordinate Systems and Transformation Matrices . . . . . . . . . 86

A.2 Absolute Displacement of the Aerodynamic Centre due to the Structural

Displacement . . . . . . . . . . . . . . . . . . . . . 88

A.3 Rotation of the Blade Section due to the Structural Displacement _ . . . 90

A.4 Absolute Velocity of the Aerodynamic Centre due to the Structural Velocity 91

$\begin{array}{ll}\text { References } & 94\end{array}$ 


\section{List of Tables}

$2.1 \quad$ Phase information of the first and second mode shapes . . . . . . . . . 37 


\section{List of Figures}

1.1 (a) A typical troposkien shape Vertical Axis Wind Turbine (VAWT) adapted from reference [1] (b) A typical Horizontal Axis Wind Turbine (HAWT) adapted from reference $[\square] \ldots \ldots \ldots . \ldots \ldots$

1.2 Life cycle cost breakdown of a typical baseline offshore wind project [2] . . 4

2.1 DOE-Sandia 17-meter Darrieus vertical axis wind turbine (a) Geometry specifications (dimensions in meter) (b) Coordinate system . . . . . . . . . 17

2.2 Blade cross section of the DOE-Sandia 17-meter vertical axis wind turbine: extruded aluminium, NACA0015 . . . . . . . . . . . . . . . . 17

2.3 (a) Components of the generalized displacement $y_{D}$ (b) Components of the generalized force $y_{t} \ldots \ldots \ldots \ldots \ldots$

2.4 Blade cross section: definitions . . . . . . . . . . . . . . 20

2.5 Variation of the first and second natural frequencies $\left(\omega_{1}, \omega_{2}\right)$ with the angular velocity of the vertical axis wind turbine $\Omega$ : no centre of mass offset $\left.x_{\alpha}=0.0\right)$, Coriolis effect excluded. (a) $K_{D D}$ included (b) $K_{D D}$ excluded . 32

2.6 First two mode shapes: angular velocity $\Omega=50.6 \mathrm{rpm}$, no centre of mass offset $\left(x_{\alpha}=0\right)$, Coriolis effect excluded. (a) Plunge displacement (b) Chordwise displacement (c) Spanwise displacement (d) Pitch displacement 34 
2.7 First two mode shapes: angular velocity $\Omega=50.6 \mathrm{rpm}, 10 \%$ centre of mass aft $\left(x_{\alpha}=-0.1\right)$, Coriolis effect excluded. (a) Plunge displacement (b) Chordwise displacement (c) Spanwise displacement (d) Pitch displacement

2.8 First two mode shapes: angular velocity $\Omega=50.6 \mathrm{rpm}$, no centre of mass offset $\left(x_{\alpha}=0\right)$, Coriolis effect included. (a) Plunge displacement (b) Chordwise displacement (c) Spanwise displacement (d) Pitch displacement 38

2.9 The fan plot of the DOE-Sandia 17-meter Darrieus vertical axis wind turbine: operating angular velocity $\Omega=50.6 \mathrm{rpm}, 10 \%$ centre of mass aft $\mathrm{d}$ $\left.x_{\alpha}=-0.1\right)$, Coriolis effect included. . . . . . . . . . . . . . . . . 39

3.1 Free vortex model conventions (a) Blade element local coordinate system

(b) Global coordinate system . . . . . . . . . . . . . . . . . . . . . 44

3.2 Vortex shedding for a single blade element $\ldots \ldots \ldots \ldots$. . . . . . 47

3.3 Induced velocity at a point by vortex filament . . . . . . . . . . . . 49

3.4 Comparison of equator forces between the FEM-Vort aerodynamic and VDART3: chord to equator ratio of $\frac{C}{R}=0.135$, height to radius ratio of $\frac{H}{R}=2$, tip speed ratio of $\frac{R \Omega 2}{U_{\infty}}=5$, FEM-Vort with fixed Reynolds number . 51

3.5 Comparison of equator forces between the FEM-Vort aerodynamic and VDART3: chord to equator ratio of $\frac{C}{B}=0.135$, height to radius ratio of $\frac{H}{R}=2$, tip speed ratio of $\frac{R \Omega 2}{U_{\infty}}=5$, FEM-Vort with variable Reynolds number 53

3.6 The FEM-Vort aerodynamic model: height $H=2 \mathrm{~m}$, equator radius $R=$ $1 \mathrm{~m}$, chord length $C^{\prime}=0.135 \mathrm{~m}$, the wind turbine angular velocity $\Omega \exists$ $45 \mathrm{rad} / \mathrm{s}$ and the wind velocity $U_{\infty}=9 \mathrm{~m} / \mathrm{s}$ (a) Variation of angle of attack (b) Variation of Reynolds number (c) Variation of lift coefficient at $A O A=10^{\circ}$ with Reynolds number $\ldots \ldots \ldots \ldots \ldots$ 
3.7 Convergence analysis: 17 meter DOE-Sandia VAWT, the wind turbine angular velocity $\Omega=50.6 \mathrm{rpm}$ and the wind velocity $U_{\infty}=22 \mathrm{mph} \mathrm{(a)}$

Number of blade elements (b) Number of time steps per revolution . . . . . 56

3.8 Verification of the parallel implementation in terms of the tangential force coefficient: comparison of the serial and the parallel (using 24 CPUs) implementations, 17 meter DOE-Sandia VAWT, 23 blade elements, 10 revoutions, 40 steps per revolution . . . . . . . . . . . . . . . . . . . . 59

3.9 Scalability of parallel implementation: 17 meter DOE-Sandia VAWT, 23 bade elements, 10 revolutions, 40 steps per revolution (a) Computer run time (b) Speedup . . . . . . . . . . . . . . . . . . . . . . 59

4.1 (a) Aerodynamic forces on the blade section (b) Rotating frame of reference 63

4.2 Comparison of the generated torque: 17-meter DOE-Sandia VAWT, operating angular velocity $\Omega=50.6 \mathrm{rpm}$ (a) Tip speed ratio $=2.18$ (b) Tip speed ratio $=2.8$ (c) Tip speed ratio $=4.36 \ldots \ldots \ldots \ldots \ldots$

4.3 The power curve of the DOE-Sandia 17 meter VAWT: operating angular

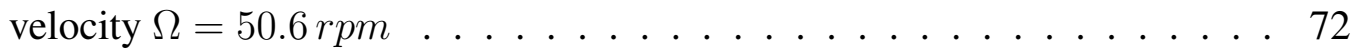

4.4 Wake structure: 17-meter DOE-Sandia VAWT, operating angular velocity $\Omega=50.6 \mathrm{rpm}$, tip speed ratio $=4.36$, azimuth angle $(\theta)=1890^{\circ} \ldots \ldots 73$

4.5 Elastic axis displacement at the equator of the 17-meter DOE-Sandia VAWT: operating angular velocity $\Omega=50.6 \mathrm{rpm}$, tip speed ratio $=4.36$ (a) Transational displacement (b) Rotational displacement . . . . . . . . . . 75

4.6 Frequency content of the elastic axis displacement at the equator of the 17 meter DOE-Sandia VAWT: operating angular velocity $\Omega=50.6 \mathrm{rpm}$, tip speed ratio $=4.36$ (a) Translational displacement (b) Pitch displacement $\ldots 75$ 
4.7 Elastic axis internal forces at the root of the 17-meter DOE-Sandia VAWT:

operating angular velocity $\Omega=50.6 \mathrm{rpm}$, tip speed ratio $=4.36$ (a) $\mathrm{Mo-}$

ment about plunge axis (b) Moment about chordwise axis (c) Axial force . . 77

4.8 Axial strain at the root of the 17-meter DOE-Sandia VAWT: operating an-

gular velocity $\Omega=50.6 \mathrm{rpm}$, tip speed ratio $=4.36$ (a) Elastic axis (b)

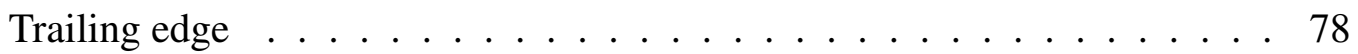

4.9 Axial stress at the root of the 17-meter DOE-Sandia VAWT: operating an-

gular velocity $\Omega=50.6 \mathrm{rpm}$, tip speed ratio $=4.36$ (a) Elastic axis (b)

Trailing edge . . . . . . . . . . . . . . . . . . 79

4.10 Elastic axis axial stress along the blades of the 17-meter DOE-Sandia VAWT:

operating angular velocity $\Omega=50.6 \mathrm{rpm}$, tip speed ratio $=4.36$, azimuth

angle $(\theta)=18909 \ldots \ldots \ldots \ldots . \ldots \ldots$

4.11 Trailing edge vibratory stress at the root of the DOE-Sandia 17-meter VAWT:

operating angular velocity $\Omega=50.6 \mathrm{rpm} \ldots \ldots \ldots$. . . . . . . 81

A.1 (a) Coordinate systems (b) Blade section displacement . . . . . . . . . . 93 


\section{Nomenclature}

\section{Latin Symbols}

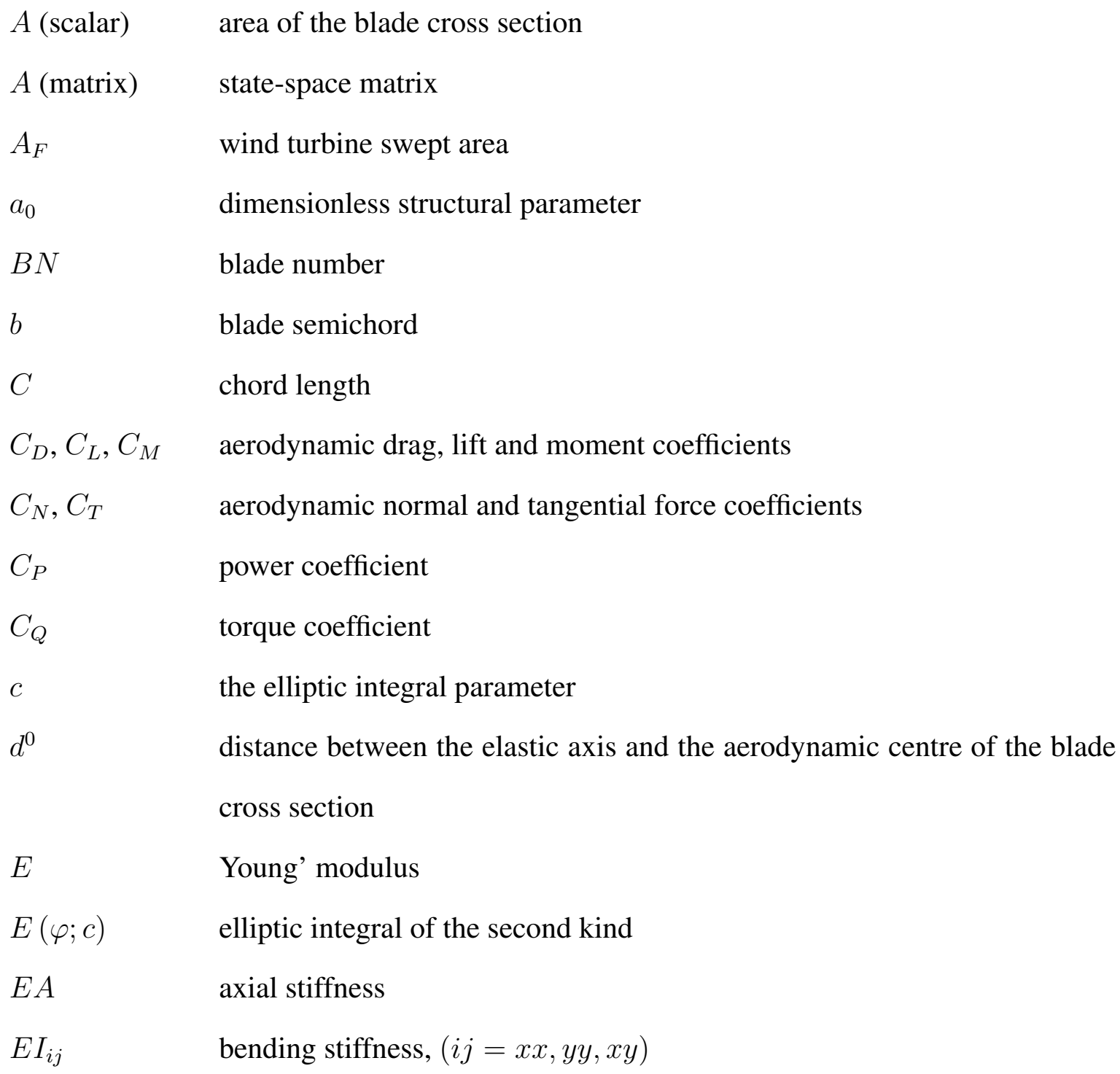




\begin{tabular}{|c|c|}
\hline $\mathbf{e}_{1}, \mathbf{e}_{2}, \mathbf{e}_{3}$ & unit triad defining the undisturbed position of the elastic axis \\
\hline $\mathbf{e}_{1}^{\prime}, \mathbf{e}_{2}^{\prime}, \mathbf{e}_{3}^{\prime}$ & unit triad defining the perturbed position of the elastic axis \\
\hline $\mathbf{e}_{\mathbf{r}}, \mathbf{e}_{\theta}, \mathbf{e}_{\zeta}$ & unit triad defining the rotating frame of reference \\
\hline$F$ & external force vector in finite element method \\
\hline$F_{N}, F_{T}$ & $\begin{array}{l}\text { total normal and tangential forces generated by the aerodynamic blade el- } \\
\text { ement }\end{array}$ \\
\hline$F(\varphi ; c)$ & elliptic integral of the first kind \\
\hline$f, f_{A}, f_{D}$ & generalized total, aerodynamic and dynamic external force vector \\
\hline$f_{D}^{1}, f_{D}^{2}, f_{D}^{2}$ & components of the dynamic force vector \\
\hline$f_{1}, f_{2}, f_{3}$ & components of the external force acting on the blade cross section \\
\hline$f_{s s}$ & generalized steady state dynamic force vector \\
\hline$f_{s s}^{i}$ & $\begin{array}{l}\text { components of the generalized steady state dynamic force vector, } \\
(i=1,2, \ldots, 6)\end{array}$ \\
\hline$G$ & shear modulus \\
\hline$G A$ & shear stiffness \\
\hline$G I_{\alpha}$ & torsional stiffness \\
\hline$H$ & total height of the blade \\
\hline$h$ & half of the total height of the blade \\
\hline $\mathrm{i}, \mathrm{j}, \mathrm{k}$ & unit triad defining the inertial reference frame \\
\hline$K_{D}$ & dynamic stiffness matrix \\
\hline$K_{D D}$ & displacement block matrix constructing the structural stiffness matrix \\
\hline$K_{D F}$ & $\begin{array}{l}\text { displacement-force coupling block matrix constructing the structural stiff- } \\
\text { ness matrix }\end{array}$ \\
\hline$K_{F D}$ & $\begin{array}{l}\text { force-displacement coupling block matrix constructing the structural stiff- } \\
\text { ness matrix }\end{array}$ \\
\hline
\end{tabular}




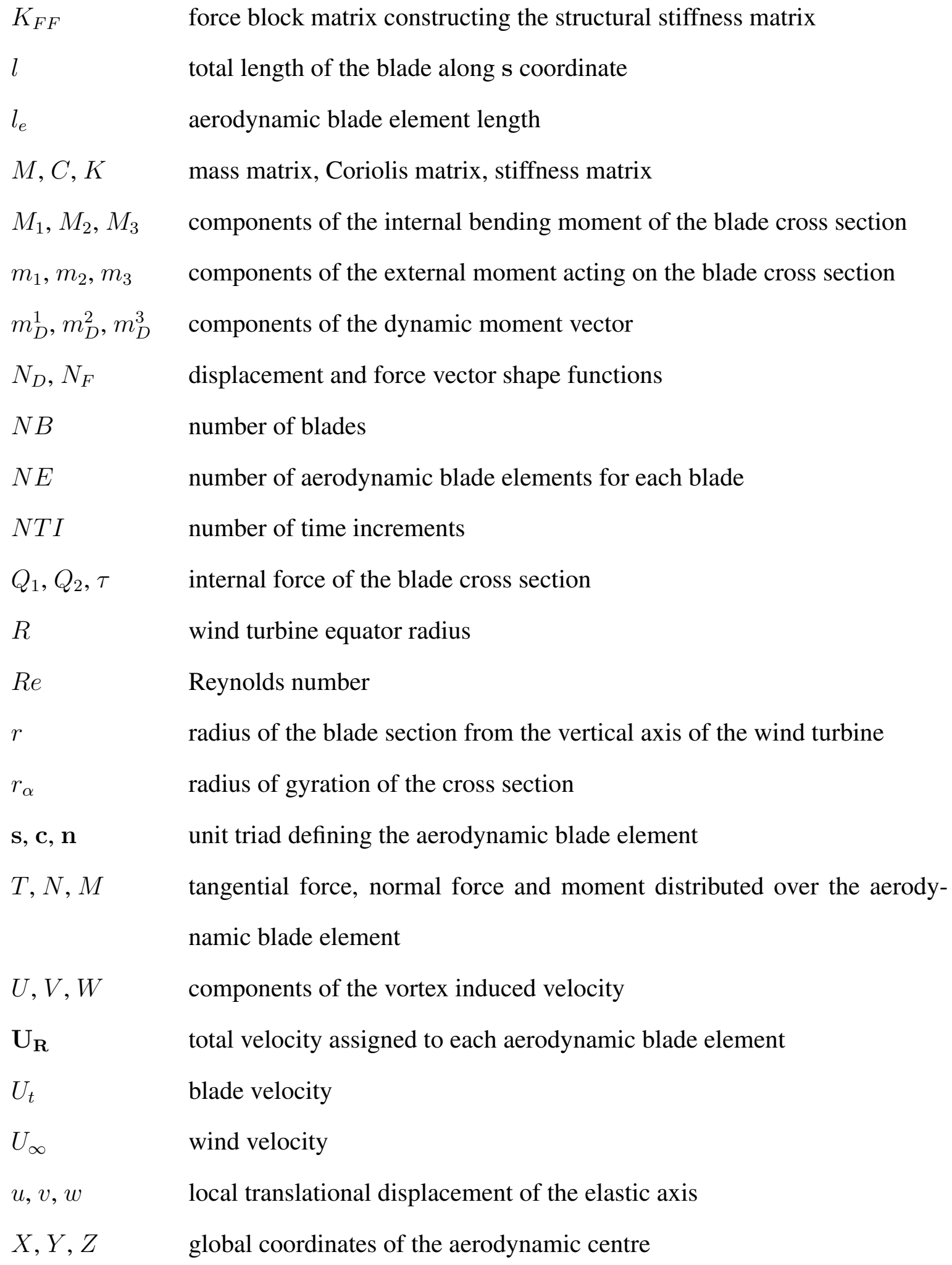




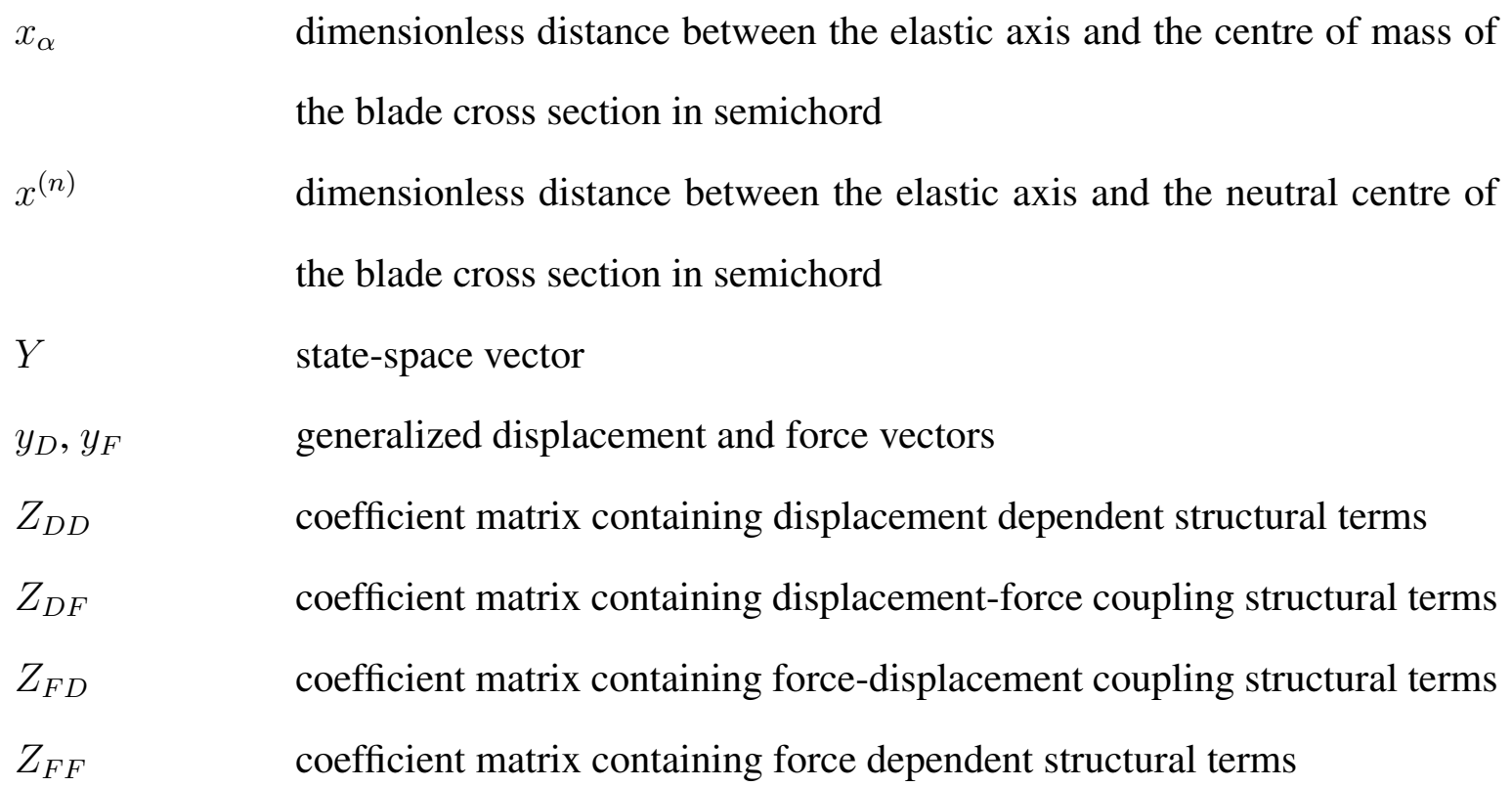

\section{Greek Symbols}

$\alpha \quad$ angle of attack

$\beta \quad$ ratio of the equator radius to half of the height of the wind turbine

$\Gamma_{B}, \Gamma_{S}, \Gamma_{T} \quad$ bound vortex, spanwise vortex, trailing tip vortex strength

$\Delta t \quad$ time increment

$\Delta X, \Delta Y, \Delta Z$ components of the aerodynamic centre movement due to the structural vibration

$\Delta c \quad$ rotation of the chordwise axis due to the structural vibration

$\Delta x, \Delta y, \Delta z \quad$ components of the vortex displacement

$\epsilon_{a c}, \epsilon_{e a} \quad$ perturbation of the aerodynamic centre and elastic axis

$\epsilon_{s s}, \sigma_{s s} \quad$ axial strain and stress

$\zeta \quad$ vertical coordinate of the troposkien

$\theta \quad$ azimuth angle

$\theta_{B} \quad$ blade azimuth angle 


$\begin{array}{ll}\kappa^{(0)} & \begin{array}{l}\text { initial local curvature of the troposkien shape } \\ \nu\end{array} \\ \rho & \text { ratio of the second moment of area about } \mathbf{e}_{1} \text { to the polar moment of area } \\ & \text { air kinematic viscosity } \\ \sigma & \text { air density } \\ \tau^{(0)}, \tau_{\text {min }}^{(0)} & \text { initial local tension of the blade and its minimum } \\ \varphi & \text { troposkien parameter } \\ \phi & \text { local angle of the troposkien } \\ \chi_{1}, \chi_{2}, \alpha & \text { local rotational displacement of the elastic axis } \\ \Omega, \Omega & \text { vector of the angular velocity of the turbine and its magnitude } \\ \omega & \text { natural frequency of the system }\end{array}$




\section{Chapter 1}

\section{Introduction}

\subsection{General}

The world is currently facing an energy dilemma, the fossil fuel is diminishing in supply while the demand for energy is increasing. This has motivated many countries to invest in harnessing energy from renewable resources. Among these resources, wind power is one of the fastest growing sectors. The most efficient approach for extracting the kinetic energy of the wind is the use of lift-driven wind turbines. These wind turbines are generally categorized into two groups: Horizontal Axis Wind Turbine (HAWT) and Vertical Axis Wind Turbine (VAWT). Fig.!.] shows a typical VAWT and HAWT. Although both wind turbines offer the same ideal efficiency [3], HAWTs hold a number of advantages over VAWTs: firstly, the HAWTs are self-starting machines, which eliminates the need for an auxiliary starter [团]. Secondly, for the same swept area, the VAWTs' blade length is two to three times greater that the HAWTs counterpart [四], and thirdly the HAWTs' aerodynamic performance is much simpler than the VAWTs [3]. The distribution of aerodynamic loading along the blades of a VAWT changes with respect to the azimuth angle; hence, leading 
to cyclic stress fatigue of the components [5]. Another complexity of the aerodynamic behaviour of VAWTs stems from the fact that the blades might encounter their wake as well as the vortices generated by other blades during one revolution. As a result, landbased HAWTs are the most commonly used wind turbines around the world.

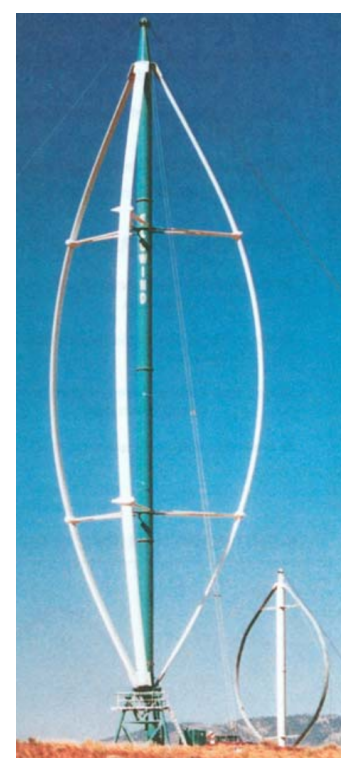

(a)

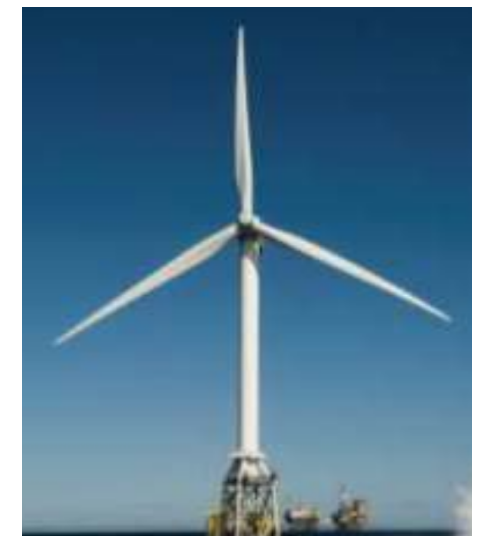

(b)

Figure 1.1: (a) A typical troposkien shape Vertical Axis Wind Turbine (VAWT) adapted from reference [四] (b) A typical Horizontal Axis Wind Turbine (HAWT) adapted from reference [[]]

As the demand for reducing the reliance on the fossil fuels increases around the world, nations are striving to find better ways to increase clean energy capacities. In the field of wind power, this has prompted the initiatives of moving wind turbines towards offshore application. For instance, in 2010, the National Renewable Energy Laboratory (NREL) of the United States estimates that U.S. offshore wind has a gross potential generating capacity four times greater than the nation's present electric capacity [2]. In general, the offshore wind power offers the following benefits []]:

- Wind speed increases with the distance from the coast significantly. This increase in 
the wind speed could lead to a $29 \%$ increase in the annual average energy production as reported by NREL.

- Less restrictive offshore transportation equipments allow installation of larger wind turbines.

- Land-based wind farms, which are typically far from the inhabited areas, require a considerable transmission capacity. Offshore wind power, which is relatively close to the coastal load centres mitigates this need; thus, it makes the energy production more cost-effective.

- With the development of the technology which facilitates the installation of wind turbines in deep water, the visible impact of wind turbine would become minimum.

- The deep water offshore installation sites also reduce the possibility of sound propagation problems in the coastal areas.

One of the major obstacles in using the offshore wind power is the higher costs associated with this technology compared to the land-based wind turbines. The existing uncertainties in the hydrodynamic loadings of offshore platforms urge the adoption of more conservative structural designs, leading to more costs. In addition, Operation and Maintenance $(\mathrm{O} \&$ M) costs in an offshore wind turbine is almost two to three times greater than a land-based wind turbine []. NREL [ [ [] $]$ gives an estimate of the life-cycle cost breakdown of a typical baseline offshore wind project, shown in Fig.[.2.

Currently, efforts are being made to reduce the total costs of offshore wind power to rationalize the use of this technology when compared to the land-based wind turbines. This effort initiated a reviving interest in VAWT offshore applications. For instance, the U.S. department of energy has put forward a five year project regarding innovative offshore 


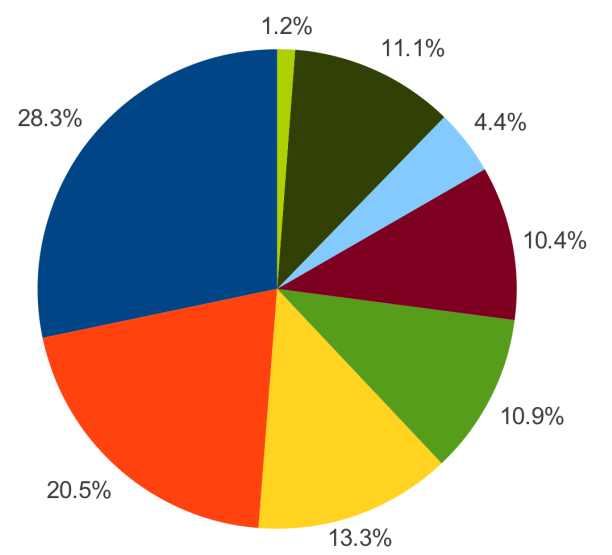

- Turbine

- Operation \& Maintenance

- Support Structures

Electrical Infrastructure

- Logistics \& Installation

Project Development \&

Permits

- Other Variable Costs

- Other Capital Costs

Figure 1.2: Life cycle cost breakdown of a typical baseline offshore wind project [2]

vertical axis wind turbine rotors pursued by Sandia National Laboratory and a number of U.S. universities [四]. This interest hinges on the advantages of VAWTs as a potential alternative to offshore HAWTs:

- VAWTs are responsive to the wind regardless of its direction. Conversely, HAWTs need to have a yawing system that adds complexity to the drive train.

- VAWTs operate at both stalled and unstalled conditions. However the stall properties of the blade removes the need for control pitch mechanism.

- Gearbox and generator of the VAWTs are placed at the bottom of the wind turbine; therefore, they are more accessible compared to HAWTs'. This valuable characteristic could reduce the cost for Operation and Maintenance $(O \& M)$ in offshore applications substantially.

- Since the heavy components of the VAWTs, generator and gearbox, are placed at the bottom of the wind turbine, the centre of gravity of the entire system is closer to the sea level. This fact helps the system to be more stable by nature, resulting in less costly support structures. 
- As mentioned earlier, one of the advantages of the offshore wind power is the possibility of building larger rotors. For HAWTs, this advantage conflicts with the fact that the larger wind turbine becomes heavier and the cyclic effect of the weight causes fatigue problems. On the contrary, weight of the blade does not generally become restrictive in the design of VAWTs.

According to the aforementioned discussions, it seems that VAWTs have the potential to outperform HAWTs especially for the offshore applications. Therefore, further research seems to be necessary to fully understand the behaviour of this type of wind turbine for offshore applications. One of the major challenges in this type of applications is with regards to the hydrodynamic loading at the base of the wind turbine. The wave induced movement of the base of the wind turbine not only affects the performance of the wind turbine but also demands specially designed support structures. The design of support structures becomes a significantly crucial step when deep-water installation is concerned. However, in light of future research efforts, better designs of this type of wind turbine could alleviate these challenges in such a way that their future use become more feasible. Perhaps, one of the very first steps aims at a deeper understanding of the land-based version of the VAWTs.

The focus of the current thesis is the study of the aeroelastic behaviour of a land-based VAWT. The type of VAWT chosen for this investigation is a Darrieus type with troposkien shape blades. Troposkien blades are referred to as the ones having the shapes that look like a rope spinning about a vertical axis while it is fixed at its ends. More details of the methodology implemented in this thesis is described in the following sections. 


\subsection{Literature Survey of the Aeroelastic Analysis of Verti- cal Axis Wind Turbines}

In general, there are two categories of aerodynamic models of VAWTs: momentum theory and vortex theory based models. The first momentum based model was proposed by Templin in 1974 [目]. In this model, it is assumed that a streamtube passes through the rotor, and inside this streamtube the induced velocity is constant everywhere. Then, the drag forces are calculated based on this constant induced velocity at the blades. Equating the drag force to the change of momentum caused by the rotor allows the calculation of the induced velocity. In order to enhance the assumption of constant induced velocity everywhere on the blade, Strickland [ $[\mathbf{D}]$ developed the multiple streamtube model, by assuming multiple streamtubes which pass through the swept volume of the rotor. Based on this assumption, the induced velocity can change at different locations along the blade. Although the multiple streamtube model is more successful in predicting the performance of the wind turbine, it still suffers from the fact that, inside one streamtube, it does not distinguish between the upwind and downwind position of the rotor. To relax this assumption, Paraschivoiu []] developed the double multiple streamtube model, where two separate sets of multiple streamtubes represent the aerodynamics of the upwind and downwind of the wind turbine. Although momentum based models are very efficient methods in terms of execution time, they inherit the deficiencies that are present in momentum theory. The root of these deficiencies originates from the fact that these models do not explicitly take into account the effect of vortices. In VAWTs, as the tip speed ratio increases, it is more likely that each blade catches with its own vortices and the ones generated by other blades. The same phenomenon occurs when more blades are added to the wind turbine, in other words, the solidity increases. In these cases, the capability of momentum based models in predicting 
not only the loads on the blade but also the performance degrades. Moreover, these models cannot provide any information about the near wake structure of the flow. This information is significantly important when the interaction of the wake with downstream wind turbine in a wind farm setting is of interest.

The second category of the VAWTs' aerodynamic models are vortex theory based models that are more computationally expensive but more accurate in estimating the aerodynamic loads in a VAWT. Strickland et al. [0] developed a three dimensional free vortex model for the study of Darrieus type VAWTs. One of the advantages of this model is that, unlike momentum based models, it fully takes into account the unsteady effects of the vortices by representing these vortices as filaments which convect freely in the fluid domain. This characteristic allows the calculation of the induced velocity everywhere in the domain, including the velocity at the blades. Therefore, this model is capable of predicting the aerodynamic loads more accurately. The strength of this method becomes even more evident when the blade-vortex interaction becomes stronger, e.g., at high tip speed ratios or high solidity ratios. As the second advantage, this model provides useful information about the near wake structure of flow downstream of the wind turbine.

Although the execution time of the free vortex method mentioned above is a lot less than a full CFD simulation, there were concerns, at the time of its development in the 1980s, regarding the restrictions that this method imposes in engineering analysis specifically due to the computer simulation run time. In an effort to tackle this challenge, Wilson and Walker [ए10] combined the streamtube model with the vortex method to reduce the computer run time. Strickland [ए]] also suggested few techniques for similar purpose at the expense of loosing the spectral accuracy to certain degrees. However, it is worth mentioning that with the current computational power available in typical laptops alongside with parallel processing capabilities such as MPI and OpenMP, the time required for these types of 
analyzes could be reduced significantly.

Besides the aerodynamic efficiency of the VAWTs, another aspect that plays an important role in justifying wind power technology as a renewable resource is relatively low cost associated with the fatigue life of the components such as the blades. Veers [5] highlights the importance of estimating the time history of stress for the fatigue analysis of the VAWTs. The need for an acceptable estimation of stress in VAWTs underlines the necessity of structural dynamic and aeroelastic analysis as a crucial part of VAWTs' study. Numerous researchers have addressed different aspects of the aeroelastic analysis of VAWTs, in particular, one could refer to the reports from the Sandia National Laboratory in the 1980s.

Lobitz [ㅁ] developed the transient dynamic analysis package (VAWTDYN) for the analysis of VAWTs, which utilizes the single streamtube aerodynamic model. VAWDYN is used to predict the vibratory stress at the root of DOE-Sandia 17-meter VAWT. The comparison of the results with the available experimental data reveals limited success mainly due to the inaccurate prediction of the loads by the single streamtube model. Watson [1]3] compares the predictions of a non-linear finite element analysis with the strain gauge measurements on the DOE-Sandia 17-meter VAWT. In this analysis, the effect of only centrifugal and gravitational forces are considered. Carne et al. [144] derived the structural dynamic equations for a rotating structure while ignoring the aerodynamic forces. They perform a modal analysis to compare the predicted mode shapes and frequencies with the experimental data available for the Sandia 2-meter vertical axis wind turbine. In their analysis, which is developed in the finite element framework, rotational degrees of freedom and their corresponding kinetic energy are neglected. Lobitz and Sullivan [ए5] apply a similar finite element derivation to evaluate the forced response of the DOE-Sandia low cost 17meter blade to the aerodynamic loading. Two aerodynamic models are utilized: the single streamtube model and the double multiple streamtube model. It is shown that although the 
agreement of the numerical results with the experimental data is encouraging, it does not absolutely predict all response details with high accuracy [ㅍ]. One of the sources of error mentioned in this report is the uncertainty in aerodynamic load prediction.

Nitzsche [ए]] distinctively derived a rigorous set of dynamic equations of motion that accounts for the Coriolis effect, centrifugal softening and centrifugal stiffening. These equations are coupled with the quasi-steady aerodynamic loading in order to assess the aeroelastic instability of the VAWTs. The aerodynamic loading which is based on the Theodorsen's theory does not take into account the stall conditions, wake effect and the unsteadiness of the flow. Popelka [ए]] also formulates a similar set of structural dynamic equations for the troposkien shape blades. In these equations, the effects of tower and the drive train are also modelled by torsional springs. In Popelka's analysis, a Theodorsen based aerodynamic model is adopted for the unsteady effects; however, the wake effect and the stall conditions are neglected.

\subsection{The Proposed Approach for Aeroelastic Analysis of Vertical Axis Wind Turbines}

As emphasized in the previous section, all of the aeroelastic analyzes performed on the VAWTs so far suffer from the lack of a robust aerodynamic modelling. They use a momentum based model combined with either the experimental data or the Theodorsen's theory. Both of these models do not take into consideration the wake effect rigorously. In an attempt to alleviate such problem, the free vortex model proposed by Strickland et al. [Q] is adopted in the current investigation. Furthermore, two new features are integrated with the original model proposed by Strickland et al. [0]:

- The original model by Strickland only uses the set of experimental data that corre- 
sponds to the equator maximum Reynolds number. This assumption is not truly valid because not only the magnitude of the blade velocity changes at different sections along the blade but also its angle with the wind velocity changes with the azimuth angle of the turbine. Therefore, the Reynolds number associated with the relative velocity changes along the blade as well as in time. In the current study, the effect of variable Reynolds number is taken into account by using interpolation between the experimental data available for a wide range of Reynolds number.

- In order to reduce the computer run time of the model, the method is parallelized by taking advantage of the shared-memory parallel programming interface, OpenMP.

The structural dynamic equations implemented in this study are the ones devised by Nitzsche [ए6]. Nitzsche represents the structural dynamic behaviour of a three dimensional linear curved beam with troposkien shape in a state vector form. This state vector consists of 12-first order linear ordinary differential equations, based on generalized force and generalized displacement variables. This formulation explicitly represents the effect of the Coriolis forces, centrifugal softening and tensile stress caused by the centrifugal forces. Nitzsche applies a frequency domain based method, namely Transfer Matrix method, to examine the stability of the system. Here, due to the mixed nature of the equations, as opposed to the irreducible form, the equations are cast into a mixed finite element formulation and the response of the system is calculated in time.

Finally, the structural dynamic equations, in mixed finite element form, are coupled with the free vortex model. The aeroelastic analysis tool, named FEM-Vort, is used to predict aerodynamic performance and structural responses of the DOE-Sandia 17-meter VAWT. The numerical results are compared with the available experimental data. 


\subsection{Objectives of the Thesis}

The objectives of the thesis are as follows:

- Aerodynamic analysis: the aerodynamic model is a combination of the free vortex model with the lift and drag coefficients obtained from the experimental data. One of the primary objectives of this study is to investigate the accuracy of this model in estimating the aerodynamic loading on the blade. Secondly, it is of interest of this study to assess the structure of the wake and its influence on the performance of the wind turbine.

- Structural dynamic analysis: the main objective of the structural dynamic analysis is to evaluate the effect of the Coriolis and centrifugal forces on the characteristic of the system.

- Aeroelastic analysis: The ultimate goal of this investigation is to predict the time history of the strain and stress along the blade at different tip speed ratio and locate the high stress part of the blade.

\subsection{Layout of the Thesis}

Chapter (2): it begins with the structural dynamic equations used in this thesis. The application of mixed finite element method is presented next. Finally, the characteristics of the system are investigated through modal analysis.

Chapter (3): this chapter starts with the theoretical background to filament based free vortex model utilized in this thesis. Then, the convergence analysis is presented. At the end, the parallel implementation of this model is briefly discussed. 
Chapter (4): the first part of this chapter is devoted to the formulation of the aeroelastic coupling between the structural dynamic equations and the aerodynamic model. In the second part of the chapter, the aerodynamic and structural performance of a VAWT are evaluated.

Chapter (5): it summarizes the features of the current investigation culminating in conclusions and possible future research directions. 


\section{Chapter 2}

\section{Structural Dynamic Analysis}

\subsection{Introduction}

Structural dynamic characteristics of a troposkien shape Vertical Axis Wind Turbine (VAWT) blade are studied in this chapter. First, the closed mathematical form of the troposkien geometry is presented. Then, the structural equations, which consists of 12 first order linear ordinary differential equations based on generalized force and generalized displacement variables, are briefly mentioned, and the application of mixed finite element is described in detail. Next, the structural equilibrium equations are coupled with the dynamic equations, arising from the rotation of the blade, to form a complete set of structural dynamic equations in the mixed finite element framework. Finally, the natural frequencies and mode shapes of the system are obtained to explain the underlying physics of the problem. It is shown how the tension that develops in the troposkien blade prevents the dynamic instability of the system. It is also demonstrated how the centre of mass offset and Coriolis forces cause the coupling between the different degrees of freedom.

The subsequent sections of this chapter closely follow the references [ए]] and [ए8]. 


\subsection{Troposkien Geometry}

Troposkien, which is originally a Greek word, refers to the shape of a rope fixed at its ends, spinning about the axis which goes through the ends [एव]. Blackwell and Reis [एव] derived the closed mathematical form of the troposkien shape. By balancing the centrifugal forces and the tension that develops along the rope, a unique mathematical formula is obtained as

$$
\frac{l}{2 h}=\frac{2}{1-c^{2}} \frac{E\left(\frac{\pi}{2} ; c\right)}{F\left(\frac{\pi}{2} ; c\right)}-1
$$

where $l$ is the total length of the blade, $h$ is half of the total height of the blade, and $F\left(\frac{\pi}{2} ; c\right)$ and $E\left(\frac{\pi}{2} ; c\right)$ are the complete elliptical integral of first and second type with the parameter $c$, defined as

$$
F(\varphi ; c)=\int_{0}^{\varphi} \frac{d \vartheta}{\sqrt{1-c^{2} \sin ^{2} \vartheta}} \text { and } E(\varphi ; c)=\int_{0}^{\varphi} \sqrt{1-c^{2} \sin ^{2} \vartheta} d \vartheta
$$

Substituting a known value of the non-dimensional rope length $(l / 2 h)$ into Eq.2.Dresults in a unique value of $c$. It can be shown that when the non-dimensional length $(l / 2 h)$ changes from 1 to 5 , the parameter $c$ varies from 0 to 0.9 . Following the evaluation of $c$, one can find the rotational parameter $\theta$, and the parameter $\beta$ as

$$
\theta=\sqrt{1-c^{2}} F\left(\frac{\pi}{2} ; c\right) \text { and } \beta=\frac{2 c}{\theta \sqrt{1-c^{2}}}
$$

Since $\beta=\frac{R}{h}$, finding $\beta$ determines the value of equator radius $R$. In order to completely define the troposkien shape, assume $\varphi=\sin ^{-1}\left(\frac{r}{R}\right)$, where $r$ is the radius of the troposkien blade at each section from the vertical axis and varies from zero at the end supports to its maximum at the equator. Then, corresponding to this $r$ value, the height of the section $\zeta$, 
which changes from 0 to $h$, can be calculated as [प्प]

$$
\frac{\zeta}{h}=1-\frac{F(\varphi ; c)}{F\left(\frac{\pi}{2} ; c\right)}
$$

There are three important parameters related to the troposkien shape that will be used often in the following sections: initial axial stress $\tau^{(0)}$, the initial curvature $\kappa^{(0)}$ and the angle $\phi$. Here is a brief description of these parameters:

- Initial axial stress $\tau^{(0)}$ : due to the centrifugal forces caused by the rotation of the turbine, axial stress develops along the blade. This axial tension changes the bending stiffness of the blade; hence, it has to be added to the stiffness matrix of the system. The value of this stress, which is proportional to the square of the angular velocity of the turbine $\Omega$, becomes minimum at the equator with a value of [प्प]

$$
\tau_{\min }^{(0)}=\sigma A \frac{\Omega^{2} h^{2}}{\theta^{2}},
$$

where $\sigma A$ is the mass per unit span of the blade. The initial axial tension at other sections along the blade can be found from the following equation [एव]

$$
\frac{\tau^{(0)}}{\tau_{\min }^{(0)}}=1-\frac{\theta^{2} \beta^{2}}{2}\left(\frac{r^{2}}{R^{2}}-1\right) .
$$

This equation reveals that the maximum axial tension occurs at both ends of the blade.

- Initial local curvature of the blade $\kappa^{(0)}$ : in a troposkien shape, $\kappa^{(0)}$ near the ends is close to zero, because the blade resembles a straight line. In the middle region, where the shape is almost circular, the initial curvature $\kappa^{(0)}$ is the inverse of the local radius. 
- Angle $\phi$ : this angle, shown in Fig.R.].a, is defined based on the rotating frame of reference $\left\langle\mathbf{e}_{1}, \mathbf{e}_{2}, \mathbf{e}_{3}\right\rangle$. The origin of this rotating frame of reference lies on the elastic axis (shear centre) of the cross section of the blade. This coordinate system will be described in more detail in the following section. Note that the values of $\phi$ are less than $90^{\circ}$ in the upper half of the troposkien shape and greater than $90^{\circ}$ in the lower half.

Since any point on a perfect troposkien shape blade has its own local curvature that is different from the local curvatures of the other points on the blade, manufacturing a true troposkien blade is not practically feasible. Hence, Sandia designed a shape that is very similar to a perfect troposkien shape but is more feasible to build. Sandia used this shape in creating the blades of the DOE-Sandia 17-meter VAWTs in 1979. It consists of a middle circular segment that is connected to the supports by two straight parts. Fig.2.].b shows the details of this shape. The middle circular segment has a radius of $5.64 \mathrm{~m}$, and the two straight segments have a length of $6.21 \mathrm{~m}$. The cross section of the blade of the DOESandia 17-meter VAWT is illustrated in Fig.2.2. The cross section is an extruded aluminium section with NACA0015 shape and $0.61 \mathrm{~m}$ chord length.

\subsection{Structural Analysis}

\subsubsection{Structural Equilibrium Equations}

Nair and Hegemier [20] cast the structural equilibrium equations of a three-dimensionally curved beam in 12 first order differential equations in terms of generalized displacements and forces. Nitzsche [[0] simplifies these equations particularly for the troposkien shape blades. The first six equations, which contain the first derivatives of the generalized displacements, i.e., $y_{D}=\left\{u \chi_{1} v \chi_{2} w \alpha\right\}^{T}$ with respect to the spatial coordinate $s$ along the 


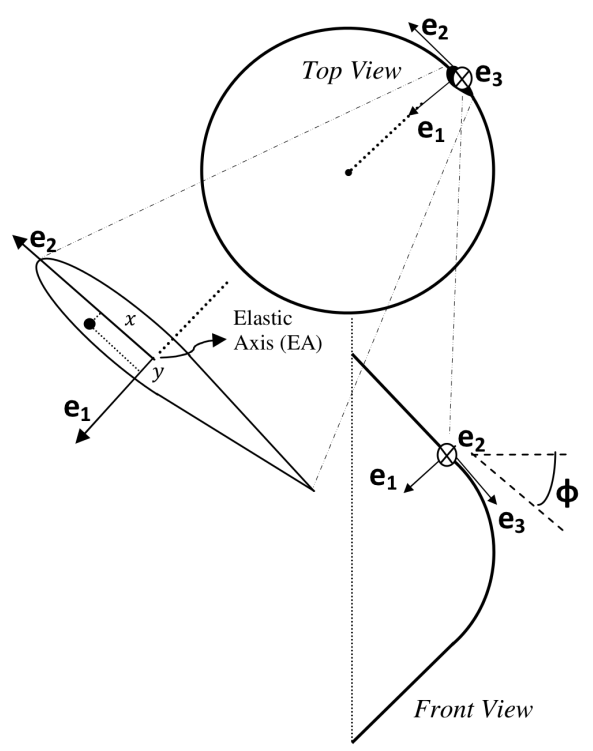

(a)

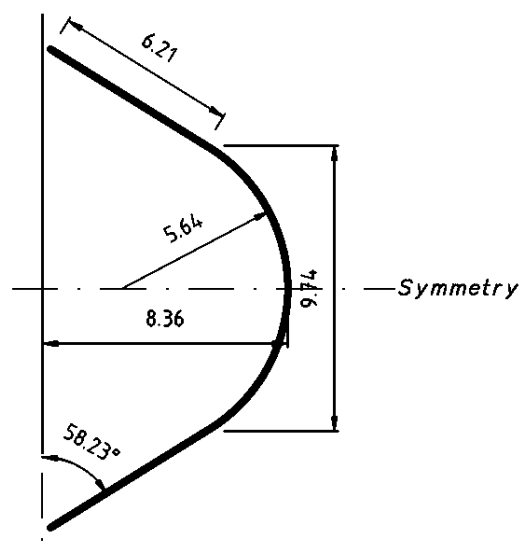

Figure 2.1: DOE-Sandia 17-meter Darrieus vertical axis wind turbine (a) Geometry specifications (dimensions in meter) (b) Coordinate system

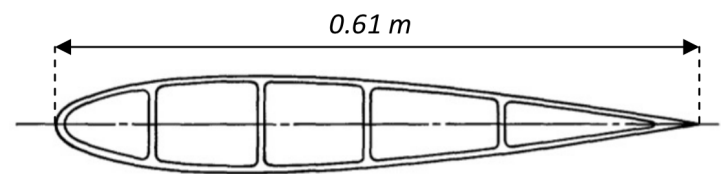

Figure 2.2: Blade cross section of the DOE-Sandia 17-meter vertical axis wind turbine: extruded aluminium, NACA0015 
blade, are represented as

$$
\begin{aligned}
& u^{\prime}+\kappa^{(0)} w-\chi_{2}-\frac{Q_{1}}{G A}=0, \\
& \chi_{1}^{\prime}+\kappa^{(0)} \alpha-a_{0} \frac{M_{1}}{E I_{y y}}+b x^{(n)} a_{0} \frac{\tau}{E I_{y y}}=0, \\
& v^{\prime}+\chi_{1}-\frac{Q_{2}}{G A}=0 \\
& \chi_{2}^{\prime}-\frac{M_{2}}{E I_{x x}}=0 \\
& w^{\prime}-\kappa^{(0)} u-a_{0} \frac{\tau}{E A}+b x^{(n)} a_{0} \frac{M_{1}}{E I_{y y}}=0, \\
& \alpha^{\prime}-\kappa^{(0)} \chi_{1}-\frac{M_{3}}{G I_{\alpha}}=0
\end{aligned}
$$

and the second six, which are based on the first derivatives of the generalized forces, i.e., $y_{F}=\left\{Q_{1} M_{1} Q_{2} M_{2} \tau M_{3}\right\}^{T}$, are

$$
\begin{aligned}
& Q_{1}^{\prime}+\kappa^{(0)} \tau+f_{1}=0, \\
& M_{1}^{\prime}+\kappa^{(0)} M_{3}-Q_{2}-\tau^{(0)} \chi_{1}+m_{1}=0, \\
& Q_{2}^{\prime}+f_{2}=0 \\
& M_{2}^{\prime}+Q_{1}-\tau^{(0)} \chi_{2}+m_{2}=0, \\
& \tau^{\prime}-\kappa^{(0)} Q_{1}+f_{3}=0 \\
& M_{3}^{\prime}-\kappa^{(0)} M_{1}+m_{3}=0 .
\end{aligned}
$$

Note that the generalized displacements $y_{D}$ consists of the translational displacement $\left(u \mathbf{e}_{1}+v \mathbf{e}_{2}+w \mathbf{e}_{3}\right)$ and the rotational displacement $\left(\chi_{1} \mathbf{e}_{1}+\chi_{2} \mathbf{e}_{2}+\alpha \mathbf{e}_{3}\right)$. Similarly the generalized force $y_{F}$ is combined of the translational force vector $\left(Q_{1} \mathbf{e}_{1}+Q_{2} \mathbf{e}_{2}+\tau \mathbf{e}_{\mathbf{3}}\right)$ and the moment vector $\left(M_{1} \mathbf{e}_{1}+M_{2} \mathbf{e}_{2}+M_{3} \mathbf{e}_{3}\right)$. These vectors are demonstrated in Fig.2.3. 


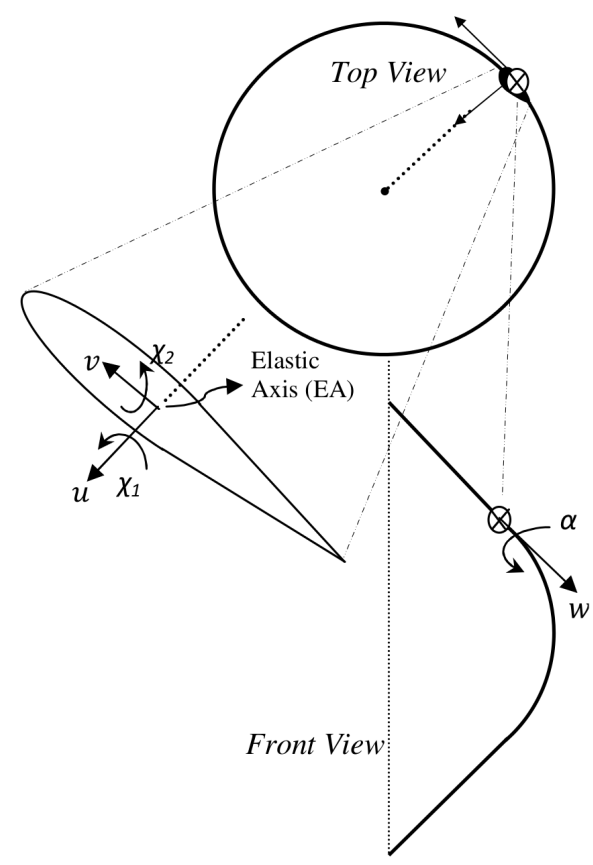

(a)

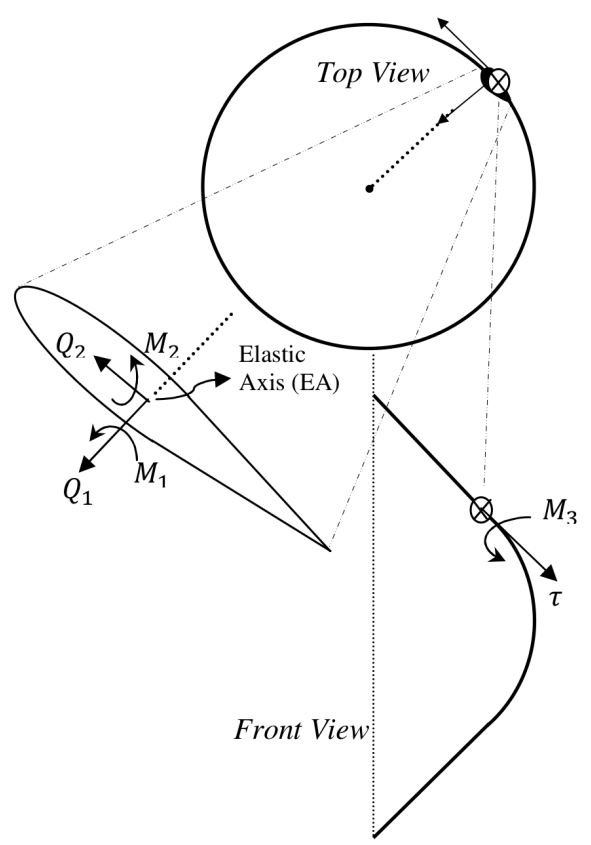

(b)

Figure 2.3: (a) Components of the generalized displacement $y_{D}$ (b) Components of the generalized force $y_{F}$

The rotating frame of reference $\left\langle\mathbf{e}_{1}, \mathbf{e}_{2}, \mathbf{e}_{3}\right\rangle$ is shown in Fig.2.].b. In this frame of reference:

- $\mathbf{e}_{1}$ is towards the local curvature.

- $\mathbf{e}_{2}$ is along the chord towards the leading edge.

- $\mathbf{e}_{3}$ is the cross product of the other two unit vectors, i.e., $\mathbf{e}_{\mathbf{3}}=\mathbf{e}_{1} \times \mathbf{e}_{\mathbf{2}}$.

It is worth emphasizing that, in Eq.2.7] and Eq.2.8, both $\kappa^{(0)}$ and $\tau^{(0)}$, which are the curvature and normal stress along the blade, vary with respect to the spatial coordinate $s$. In the aforementioned equations, $x^{(n)}$, the distance between the shear centre and the neutral 
centre, and $a_{0}$ are defined as

$$
x^{(n)}=\frac{1}{b E A} \int_{A} E x d A \quad \text { and } \quad a_{0}=\frac{1}{1-\left(b x^{(n)}\right)^{2} \frac{E A}{E I_{y y}}},
$$

where $b$ is the half chord length. Refer to Fig. 2.4 for the definition of $x^{n}$. Note that any point on the cross section is defined as $\left(y \mathbf{e}_{1}+x \mathbf{e}_{2}\right)$, and, $I_{x x}$ and $I_{y y}$ are the second moment of area about $\mathbf{e}_{2}$ and $\mathbf{e}_{1}$ respectively. Their summation is the polar moment of area denoted by $I_{\alpha}$.

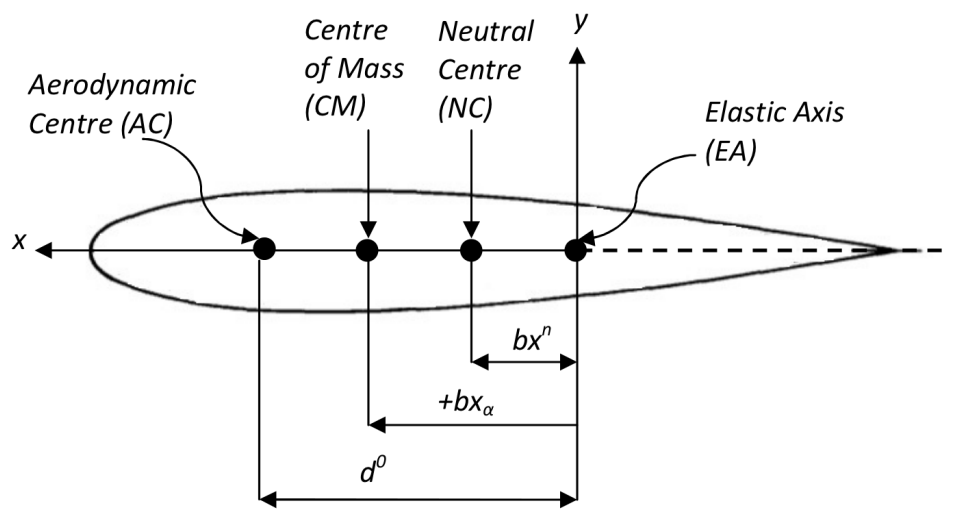

Figure 2.4: Blade cross section: definitions

For applying finite element analysis in the following section, the structural equilibrium equations, Eq.2.7 and Eq.2.8, are cast into a more suitable matrix form

$$
\frac{d}{d s}\left\{\begin{array}{l}
y_{D} \\
y_{F}
\end{array}\right\}+\left(\begin{array}{cc}
Z_{F F} & Z_{F D} \\
Z_{D F} & Z_{D D}
\end{array}\right)\left\{\begin{array}{l}
y_{F} \\
y_{D}
\end{array}\right\}+\left\{\begin{array}{l}
0 \\
f
\end{array}\right\}=\left\{\begin{array}{l}
0 \\
0
\end{array}\right\},
$$


where

$$
\begin{aligned}
& Z_{F F}=\left(\begin{array}{cccccc}
-\frac{1}{G A} & 0 & 0 & 0 & 0 & 0 \\
0 & -\frac{a_{0}}{E I_{y y}} & 0 & 0 & \frac{b x^{(n)} a_{0}}{E I_{y y}} & 0 \\
0 & 0 & -\frac{1}{G A} & 0 & 0 & 0 \\
0 & 0 & 0 & -\frac{1}{E I_{x x}} & 0 & 0 \\
0 & \frac{b x^{(n)} a_{0}}{E I_{y y}} & 0 & 0 & -\frac{a_{0}}{E A} & \\
0 & 0 & 0 & 0 & 0 & -\frac{1}{G I_{\alpha}}
\end{array}\right) \\
& Z_{D F}=-Z_{F D}^{T}=\left(\begin{array}{cccccc}
0 & 0 & 0 & -1 & \kappa^{0} & 0 \\
0 & 0 & 0 & 0 & 0 & \kappa^{0} \\
0 & 1 & 0 & 0 & 0 & 0 \\
0 & 0 & 0 & 0 & 0 & 0 \\
-\kappa^{0} & 0 & 0 & 0 & 0 & 0 \\
0 & -\kappa^{0} & 0 & 0 & 0 & 0
\end{array}\right) \text { and } Z_{D D}=\left(\begin{array}{cccccc}
0 & 0 & 0 & 0 & 0 & 0 \\
0 & -\tau^{0} & 0 & 0 & 0 & 0 \\
0 & 0 & 0 & 0 & 0 & 0 \\
0 & 0 & 0 & -\tau^{0} & 0 & 0 \\
0 & 0 & 0 & 0 & 0 & 0 \\
0 & 0 & 0 & 0 & 0 & 0
\end{array}\right),
\end{aligned}
$$

and the external force vector is given as

$$
f=\left\{f_{1} m_{1} f_{2} m_{2} f_{3} m_{3}\right\}^{T} .
$$

Eq. 2.10 is assumed to be valid in the domain $\Omega$ with the following boundary conditions

$$
y_{F}=\bar{y}_{F} \quad \text { on } \Gamma_{F} \quad \text { and } \quad y_{D}=\bar{y}_{D} \text { on } \Gamma_{D} .
$$

\subsubsection{Mixed Finite Element Formulation}

As shown in the previous section, the structural equilibrium equations of a curved beam is presented as a set of 12 first order differential equations. The number of the dependent variables in these equations, Eq.2.7 and Eq.2.8, can be reduced by suitable algebraic manipulation. Hence, this type of equations is termed mixed form [2]] as opposed to the irreducible form. Irreducible forms, compared to the mixed forms, contain less number of 
dependent variables and consequently higher order of derivatives. Therefore, in the framework of Finite Element Method (FEM), it could be inferred that the continuity restriction on the shape functions used in the irreducible forms is more restrictive. However, developing mixed finite element formulation needs special considerations as will be discussed here []] : Assuming the approximation of the state variables, $y_{F}$ and $y_{D}$, by their nodal values, and using the appropriate shape functions for both variables, one can write

$$
y_{F} \approx N_{F} \widetilde{y}_{F} \quad \text { and } \quad y_{D} \approx N_{D} \widetilde{y}_{D}
$$

Following the Galerkin weighted residual approach, the weighting functions (test functions) are chosen to be the same as the shape functions. Note that the essential (displacement) boundary condition $y_{D}=\bar{y}_{D}$ is satisfied by the appropriate selection of the shape functions, $N_{D}$. Multiplying the first equation of the system of equations presented in Eq. 2.10 by the appropriate shape function $N_{F} \delta \widetilde{y}_{F}$, the first weighted statement is obtained as

$$
\int_{\Omega} \delta \widetilde{y}_{F}^{T} N_{F}^{T}\left(\frac{d}{d s}\left(N_{D} \widetilde{y}_{D}\right)+Z_{F F}\left(N_{F} \widetilde{y}_{F}\right)+Z_{F D}\left(N_{D} \widetilde{y}_{D}\right)\right) d \Omega=0
$$

where $\Omega$ is the finite element domain. Eliminating the arbitrary parameter $\delta \widetilde{y}_{F}^{T}$ and rearranging the terms result in the following equation

$$
\left(\int_{\Omega} N_{F}^{T} Z_{F F} N_{F} d \Omega\right) \widetilde{y}_{F}+\left(\int_{\Omega} N_{F}^{T}\left(\frac{d N_{D}}{d s}+Z_{F D} N_{D}\right) d \Omega\right) \widetilde{y}_{D}=0
$$

The second equation of the same system, augmented by the natural (force) boundary con- 
dition $y_{F}=\bar{y}_{F}$ and multiplied by the shape function $N_{D} \delta \widetilde{y}_{D}$, is represented as

$$
\begin{aligned}
& -\int_{\Omega} \delta \widetilde{y}_{D}^{T} N_{D}^{T}\left(\frac{d}{d s}\left(N_{F} \widetilde{y}_{F}\right)+Z_{D F}\left(N_{F} \widetilde{y}_{F}\right)+Z_{D D}\left(N_{D} \widetilde{y}_{D}\right)+f\right) d \Omega \\
& +\int_{\Gamma_{F}} \delta \widetilde{y}_{D}^{T} N_{D}^{T}\left(N_{F} \widetilde{y}_{F}-\bar{y}_{F}\right) d \Gamma=0
\end{aligned}
$$

where $\Gamma$ is the boundary of the finite element domain. Applying the integration by parts on the first term (gradient term) leads to

$$
\begin{aligned}
& \int_{\Omega} \delta \widetilde{y}_{D}^{T} \frac{d N_{D}^{T}}{d s} N_{F} \widetilde{y}_{F} d \Omega-\int_{\Gamma} \delta \widetilde{y}_{D}^{T} N_{D}^{T} N_{F} \widetilde{y}_{F} d \Gamma-\int_{\Omega} \delta \widetilde{y}_{D}^{T} N_{D}^{T} Z_{D F} N_{F} \widetilde{y}_{F} d \Omega \\
& -\int_{\Omega} \delta \widetilde{y}_{D}^{T} N_{D}^{T} Z_{D D} N_{D} \widetilde{y}_{D} d \Omega-\int_{\Omega} \delta \widetilde{y}_{D}^{T} N_{D}^{T} f d \Omega+\int_{\Gamma_{F}} \delta \widetilde{y}_{D}^{T} N_{D}^{T} N_{F} \widetilde{y}_{F} d \Gamma \\
& -\int_{\Gamma_{F}} \delta \widetilde{y}_{D}^{T} N_{D}^{T} \bar{y}_{F} d \Gamma=0
\end{aligned}
$$

and expanding the second term on both boundaries results in

$$
\begin{aligned}
& \int_{\Omega} \delta \widetilde{y}_{D}^{T} \frac{d N_{D}^{T}}{d s} N_{F} \widetilde{y}_{F} d \Omega-\int_{\Gamma_{F}} \delta \widetilde{y}_{D}^{T} N_{D}^{T} N_{F} \widetilde{y}_{F} d \Gamma-\int_{\Gamma_{D}} \delta \widetilde{y}_{D}^{T} N_{D}^{T} N_{F} \widetilde{y}_{F} d \Gamma \\
& -\int_{\Omega} \delta \widetilde{y}_{D}^{T} N_{D}^{T} Z_{D F} N_{F} \widetilde{y}_{F} d \Omega-\int_{\Omega} \delta \widetilde{y}_{D}^{T} N_{D}^{T} Z_{D D} N_{D} \widetilde{y}_{D} d \Omega-\int_{\Omega} \delta \widetilde{y}_{D}^{T} N_{D}^{T} f d \Omega \\
& +\int_{\Gamma_{F}} \delta \widetilde{y}_{D}^{T} N_{D}^{T} N_{F} \widetilde{y}_{F} d \Gamma-\int_{\Gamma_{F}} \delta \widetilde{y}_{D}^{T} N_{D}^{T} \bar{y}_{F} d \Gamma=0 .
\end{aligned}
$$

In the above equation, the second and seventh terms cancel out, the third term vanishes since $\delta \widetilde{y}_{D}^{T}$ is zero on $\Gamma_{D}$, and $\delta \widetilde{y}_{D}^{T}$ can be eliminated in the other terms because its an arbitrary parameter. Hence, the simplified version of the aforementioned equation is given 
as

$$
\begin{aligned}
& \left(\int_{\Omega}\left(\frac{d N_{D}^{T}}{d s}-N_{D}^{T} Z_{D F}\right) N_{F} d \Omega\right) \widetilde{y}_{F}-\left(\int_{\Omega} N_{D}^{T} Z_{D D} N_{D} d \Omega\right) \widetilde{y}_{D} \\
& -\int_{\Omega} N_{D}^{T} f d \Omega-\int_{\Gamma_{F}} N_{D}^{T} \bar{y}_{F} d \Gamma=0 .
\end{aligned}
$$

Finally, combining Eq.[.]3] and Eq. 2.55 gives rise to the discretized version of Eq. 2.10

$$
\left(\begin{array}{cc}
K_{F F} & K_{F D} \\
K_{D F} & K_{D D}
\end{array}\right)\left\{\begin{array}{c}
\widetilde{y}_{F} \\
\widetilde{y}_{D}
\end{array}\right\}+\left\{\begin{array}{l}
0 \\
F
\end{array}\right\}=\left\{\begin{array}{l}
0 \\
0
\end{array}\right\}
$$

where

$$
\begin{aligned}
& K_{F F}=\int_{\Omega} N_{F}^{T} Z_{F F} N_{F} d \Omega, \quad K_{F D}=\int_{\Omega} N_{F}^{T} \frac{d N_{D}}{d s} d \Omega+\int_{\Omega} N_{F}^{T} Z_{F D} N_{D} d \Omega, \\
& K_{D F}=\int_{\Omega} \frac{d N_{D}^{T}}{d s} N_{F} d \Omega-\int_{\Omega} N_{D}^{T} Z_{D F} N_{F} d \Omega, \quad K_{D D}=-\int_{\Omega} N_{D}^{T} Z_{D D} N_{D} d \Omega, \\
& F=-\int_{\Omega} N_{D}^{T} f d \Omega-\int_{\Gamma_{F}} N_{D}^{T} \bar{y}_{F} d \Gamma .
\end{aligned}
$$

Note that both $K_{F F}$ and $K_{D D}$ are symmetric matrices, and $K_{F D}=K_{D F}^{T}$. Hence, the global stiffness matrix presented in Eq.2.16 is symmetric, which confirms that the system is indeed conservative.

Using the Schur complement of the block matrix $K_{F F}$, the system of equations presented in Eq.2.16 can still be condensed to one single equation, as follows

$$
\left(K_{D D}-K_{D F} K_{F F}^{-1} K_{F D}\right) \widetilde{y}_{D}+F=0 .
$$

Solvability of the above system is guaranteed upon the non-singularity of the coefficient matrix, $K_{D D}-K_{D F} K_{F F}^{-1} K_{F D}$. Babuska and Brezzi [22, 23], 24] derived the necessary 
and sufficient conditions for the non-singularity of this matrix. Inherent in this condition is the necessary, but not sufficient, criterion that requires the number of unknowns in the $y_{F}$ vector being equal to or greater than the number of unknowns in the vector $y_{D}$. As evident in the current investigation, this criterion is already satisfied.

\subsection{Dynamic Analysis}

In general, three types of external forces are applied on the VAWTs' blades: gravitational, aerodynamic and dynamic forces. In the current study, gravitational forces are not included. The effect of this type of forces might be significant specially for large rotors. Further research needs to be carried out to investigate such effect. The discussions related to the aerodynamic forces are presented in Chapter 3. In the following sections, the equations for dynamic forces are expressed and treated in the mixed finite element framework.

\subsubsection{Dynamic Forces}

The dynamic forces applied to a troposkien shape VAWT, which rotates about its vertical axis with the constant angular velocity, are developed by Nitzsche [ए]]. Here is a summary of the procedure: the perturbation of the centre of mass is found in a rotational frame of reference, whose origin is located at the shear centre of the blade section. Next the translational and rotational velocities are expressed in terms of this perturbation. Using the obtained velocities, the kinetic energy is calculated and used in the extended version of the Hamilton's principle. Since the potential energy is already formulated in terms of structural equilibrium equations (in Section 2.3), it is assumed that the blade is rigid; hence, its potential energy is zero. Therefore, the virtual work done by the inertial forces (in terms of the kinetic energy) balances the virtual work done by the external loads. For further details, one can refer to the cited publication. The results of this process is cast into the 
following set of equations

$$
\begin{aligned}
-\frac{f_{D}^{1}}{\sigma A} & =\ddot{u}+2 \Omega \sin \phi \dot{v}+\Omega^{2} \sin \phi g-b x_{\alpha} \ddot{\alpha}+\frac{f_{s s}^{1}}{\sigma A}, \\
-\frac{m_{D}^{1}}{\sigma A} & =b^{2}\left(x_{\alpha}^{2}+r_{\alpha}^{2}\right) \ddot{\chi_{1}}+\Omega b^{2} r_{\alpha}^{2} \sin \phi \dot{\chi_{2}}+\left(\ddot{w}-2 \Omega \cos \phi \dot{v}+\Omega^{2} \cos \phi g\right) b x_{\alpha}+\frac{f_{s s}^{2}}{\sigma A}, \\
-\frac{f_{D}^{2}}{\sigma A} & =\ddot{v}+2 \Omega \dot{g}-\Omega^{2} v+\frac{f_{s s}^{3}}{\sigma A}, \\
-\frac{m_{D}^{2}}{\sigma A} & =\left(1-\lambda^{2}\right) b^{2} r_{\alpha}^{2} \ddot{\chi}_{2}-\left(\Omega\left(\sin \phi \dot{\chi}_{1}-\cos \phi \dot{\alpha}\right)+\Omega^{2} \chi_{2}\right) b^{2} x_{\alpha}^{2}+\frac{f_{s s}^{4}}{\sigma A}, \\
-\frac{f_{D}^{3}}{\sigma A} & =\ddot{w}-2 \Omega \cos \phi \dot{v}-\Omega^{2} \cos \phi g+b x_{\alpha} \ddot{\chi}+\frac{f_{s s}^{5}}{\sigma A}, \\
-\frac{m_{D}^{3}}{\sigma A} & =b^{2}\left(x_{\alpha}^{2}+r_{\alpha}^{2}\right) \ddot{\alpha}-\Omega b^{2} r_{\alpha}^{2} \cos \phi \dot{\chi}_{1}-\left(\ddot{u}+2 \Omega \sin \phi \dot{v}+\Omega^{2} \sin \phi g\right) b x_{\alpha}+\frac{f_{s s}^{6}}{\sigma A},
\end{aligned}
$$

where

$$
g=\cos \phi\left(w+b x_{\alpha} \chi_{1}\right)-\sin \phi\left(u-b x_{\alpha} \alpha\right)
$$

and the steady state dynamic force is

$$
f_{s s}=\sigma A \Omega^{2}\left\{r \sin \phi \quad-b x_{\alpha} r \cos \phi \quad-b x_{\alpha} \quad 0 \quad-r \cos \phi \quad-b x_{\alpha} r \sin \phi\right\}^{T}
$$

One can present the aforementioned equations in a matrix form, given by

$$
-f_{D}=\bar{M} \ddot{y}_{D}+\bar{C} \dot{y}_{D}+\bar{K}_{D} y_{D}+f_{s s}
$$

where

$$
f_{D}=\left\{\begin{array}{llllll}
f_{D}^{1} & m_{D}^{1} & f_{D}^{2} & m_{D}^{2} & f_{D}^{3} & m_{D}^{3}
\end{array}\right\}^{T}
$$




$$
\bar{M}=\sigma A\left(\begin{array}{cccccc}
1 & 0 & 0 & 0 & 0 & -b x_{\alpha} \\
0 & b^{2}\left(x_{\alpha}^{2}+r_{\alpha}^{2}\right) & 0 & 0 & b x_{\alpha} & 0 \\
0 & 0 & 1 & 0 & 0 & 0 \\
0 & 0 & 0 & \left(1-\lambda^{2}\right) b^{2} r_{\alpha}^{2} & 0 & 0 \\
0 & b x_{\alpha} & 0 & 0 & 1 & 0 \\
-b x_{\alpha} & 0 & 0 & 0 & 0 & b^{2}\left(x_{\alpha}^{2}+r_{\alpha}^{2}\right)
\end{array}\right),
$$

and

$$
\bar{K}_{D}=\sigma A \Omega^{2}\left(\begin{array}{cccccc}
b x_{\alpha} \sin ^{2} \phi & b x_{\alpha} \sin \phi \cos \phi & 0 & 0 & \sin \phi \cos \phi & b x_{\alpha} \sin ^{2} \phi \\
b x_{\alpha} \sin \phi \cos \phi & -b^{2} x_{\alpha}^{2} \cos ^{2} \phi & 0 & 0 & -b x_{\alpha} \cos ^{2} \phi & -b^{2} x_{\alpha}^{2} \sin \phi \cos \phi \\
0 & 0 & -1 & 0 & 0 & 0 \\
0 & 0 & 0 & -b^{2} x_{\alpha}^{2} & 0 & 0 \\
\sin \phi \cos \phi & -b x_{\alpha} \cos ^{2} \phi & 0 & 0 & -\cos ^{2} \phi & -b x_{\alpha} \cos \phi \sin \phi \\
b x_{\alpha} \sin ^{2} \phi & -b^{2} x_{\alpha}^{2} \sin \phi \cos \phi & 0 & 0 & -b x_{\alpha} \cos \phi \sin \phi & -b^{2} x_{\alpha}^{2} \sin ^{2} \phi
\end{array}\right) .
$$

In the above matrices, $\phi$ is the angle explained in the previous sections, $b x_{\alpha}$ is the distance from the centre of mass to the shear centre of the section (positive from trailing edge to the leading edge of the section, shown in Fig.(2.4), $b r_{\alpha}$ is the radius of gyration, and $\lambda$ is the ratio of the second moment of area about the axis $\mathbf{e}_{1}$ to the polar mass moment of inertia $\lambda=\frac{I_{y y}}{I_{\alpha}}$, a value which is very close to 1 . 


\subsubsection{Mixed Finite Element Formulation}

In order to take into account the aerodynamic and dynamic forces in Eq.2.17, one can substitute $f=f_{A}+f_{D}$ into the first term of $F$ in Eq.2.16

$$
F=-\int_{\Omega} N_{D}^{T}\left(f_{A}+f_{D}\right) d \Omega-\int_{\Gamma_{F}} N_{D}^{T} \bar{y}_{F} d \Gamma
$$

Replacing $f_{D}$ by Eq. 2.20 and using $y_{D} \approx N_{D} \widetilde{y}_{D}$ results in

$$
\begin{aligned}
F & =\left(\int_{\Omega} N_{D}^{T} \bar{M} N_{D} d \Omega\right) \ddot{\widetilde{y}}_{D}+\left(\int_{\Omega} N_{D}^{T} \bar{C} N_{D} d \Omega\right) \dot{\tilde{y}}_{D}+\left(\int_{\Omega} N_{D}^{T} \bar{K}_{D} N_{D} d \Omega\right) \widetilde{y}_{D} \\
& +\int_{\Omega} N_{D}^{T} f_{s s} d \Omega-\int_{\Omega} N_{D}^{T} f_{A} d \Omega-\int_{\Gamma_{F}} N_{D}^{T} \bar{y}_{F} d \Gamma
\end{aligned}
$$

Finally, substituting back $F$ in Eq.2.17, leads to the final form of the discretized structural dynamic equation

$$
M \ddot{\widetilde{y}}_{D}+C \dot{\widetilde{y}}_{D}+K \widetilde{y}_{D}=F
$$

where $K$ is the stiffness matrix

$$
K=K_{D}+K_{D D}-K_{D F} K_{F F}^{-1} K_{F D}
$$

$M, C$ and $K_{D}$ are mass, Coriolis and centrifugal softening matrices respectively

$$
M=\int_{\Omega} N_{D}^{T} \bar{M} N_{D} d \Omega \quad C=\int_{\Omega} N_{D}^{T} \bar{C} N_{D} d \Omega \quad K_{D}=\int_{\Omega} N_{D}^{T} \bar{K}_{D} N_{D} d \Omega
$$


and the redefined external force $F$ is

$$
F=\int_{\Omega} N_{D}^{T} f_{A} d \Omega-\int_{\Omega} N_{D}^{T} f_{s s} d \Omega+\int_{\Gamma_{F}} N_{D}^{T} \bar{y}_{F} d \Gamma .
$$

With regards to Eq.2.23, it is worthwhile pointing out that:

- The mass matrix $M$ is symmetric, but the matrix $C$, which represents the Coriolis forces in the rotating frame of reference, is skew-symmetric. The skew-symmetry of the velocity-dependent matrix $C$ complies with the fact that, due to the lack of damping mechanism, the system is conservative. The Coriolis matrix $C$ gives rise to some special characteristics of the system that will be discussed thoroughly in the next section.

- Since the equations are cast in a rotating frame of reference, the matrix $K_{D}$ appears explicitly in Eq.2.23]. This matrix has a softening effect; hence, it is called centrifugal softening matrix. On the contrary, the matrix $K_{D D}$ which represents the initial tension due to the centrifugal forces, helps stiffening the blade; therefore, it is referred to as centrifugal stiffening matrix. The influence of these matrices on the structural dynamic characteristics of the system is discussed in more detail in Section 2.5.1.

The structural dynamic equation, Eq.2.23, developed here is the main equation which will be solved numerically in time in FEM-Vort. This process will be discussed in Chapter 4 after presenting the aerodynamic force calculations in Chapter 3. However, for the purpose of validating the above FEM formulation and understanding the physics of the problem, these equations are treated using eigenvalue analysis in the following section. 


\subsection{Results and Discussions}

The main focus of the current section is on the understanding of the vibration characteristics of a VAWT through eigenvalue analysis. In order to find the eigenvalues and eigenvectors of the system presented in Eq.2.23, it is first converted into a state-space form, given by

$$
\dot{Y}=A Y
$$

where $Y=\left\{\begin{array}{ll}\widetilde{y}_{D} & \dot{\widetilde{y}}_{D}\end{array}\right\}^{T}$ and

$$
A=\left(\begin{array}{cc}
0 & I \\
-M^{-1} K & -M^{-1} C
\end{array}\right) \text {. }
$$

Trying the solution $v e^{\lambda t}$, leads to the following eigenvalue problem

$$
A v=\lambda v,
$$

where $\lambda$ is the eigenvalue and $v$ is the corresponding eigenvector. It is worthwhile pointing out that a convergence analysis was carried out to investigate the sensitivity of the solution to the number of elements. It was found out that the finite element discretization is not very sensitive to the number of elements, in other words, considering only a few elements could capture the physics of the problem accurately. For the modal analysis, which will be presented in the following section, 368 one dimensional elements with linear shape functions are employed. Although using 368 elements is well beyond the required number of elements, it provides better resolution of the solution when aeroelastic analysis is of interest.

Note that the results presented in the following sections are verified against the ones ob- 
tained by applying a frequency domain based method, namely the Transfer Matrix Method, reported in reference [ए]].

\subsubsection{Coriolis Force Excluded}

\section{Softening \& Stiffening Effects}

In this part of the discussion, it is hypothetically assumed that the Coriolis force, which manifest itself in the matrix $C$, is absent. In such a case, the eigenvalues are purely imaginary numbers, and the eigenvectors are real. The first and second natural frequencies of the system are shown in the fan plot of Fig.2.5. a. As evident in this figure, both frequencies increase by the increase of the angular velocity of the wind turbine. This characteristic reveals the fact that the system is not susceptible to dynamic instability. This type of instability, which is a well-known phenomenon in rotating machinery, arises from softening stiffness. In other words, at relatively high angular velocity, the rotating machinery might experience a zero-frequency mode of vibration with positive damping. For the system undertaken investigation here, it seems that such instability is not probable. It could be demonstrated that the stability of this system mainly originates from the axial stress $\left(\tau^{(0)}\right)$ results from the centrifugal forces. This is carried out by eliminating the matrix $K_{D D}$, which accounts for the effect of the initial stress, from Eq.2.23]. Fig.2.5. b shows the variation of the first and second frequencies in this case. Illustrated in this figure, both frequencies decrease as the velocity of the wind turbine increases, until the first frequency becomes zero at about $100 \mathrm{rpm}$. At this rpm, the matrix $K_{D}$ softens the system in such a manner that the first mode becomes a rigid body mode with positive damping. The positive damping gives rise to a destabilized system whose response to any small perturbation is catastrophic. Therefore, referring again to Fig.2.5.a, one could infer that as the angular velocity of the wind 
turbine $(\Omega)$ increases, the developing axial stress $\left(\tau^{(0)}\right)$ stiffens the blade; consequently, it leads to a more stable system. Note that such a system vibrates with a higher frequency.

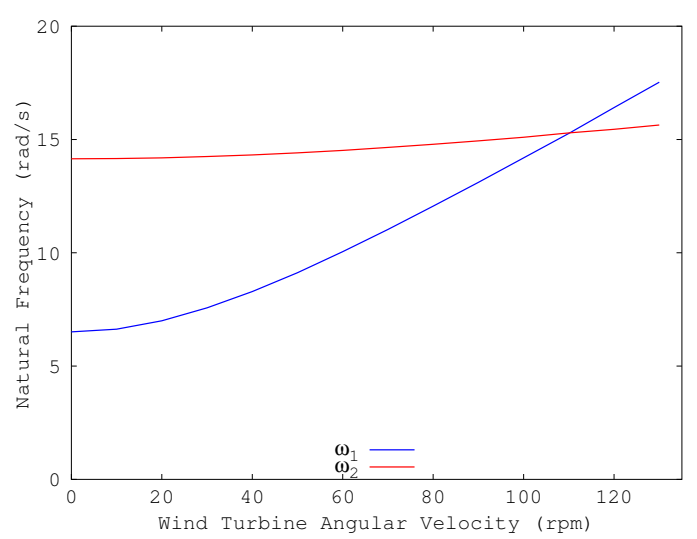

(a)

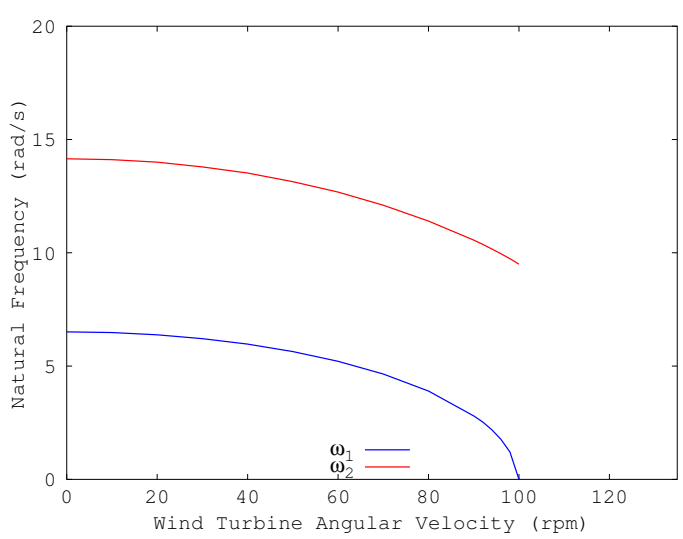

(b)

Figure 2.5: Variation of the first and second natural frequencies $\left(\omega_{1}, \omega_{2}\right)$ with the angular velocity of the vertical axis wind turbine $\Omega$ : no centre of mass offset ( $x_{\alpha}=0.0$ ), Coriolis effect excluded. (a) $K_{D D}$ included (b) $K_{D D}$ excluded

\section{Centre of Mass Offset}

The remaining part of this section aims at studying the centre of mass offset and its influence on the structural dynamic characteristics of the VAWT considered here. To accomplish this, the first and second mode shapes of the system are plotted in Fig. $\mathbf{2 . 6}$, where the centre of mass coincides with the shear centre of the cross section, i.e., $b x_{\alpha}=0$. This figure shows that the first mode shape is only a combination of an antisymmetric plunge and a symmetric spanwise displacement, while the other two degrees of freedom, the so-called chordwise and pitch degrees of freedom, are absent. On the contrary, the second mode shape only consists of symmetric chordwise and pitch displacements. For the purpose of comparison, a case is considered in which the centre of mass is located $10 \%$ aft of the elas- 
tic axis of the section. The first and second mode shapes of this system are also plotted in Fig.2.7. By comparing Fig.2.6 with Fig.[2.7, one can come to the conclusion that the centre of mass offset excites the previously absent degrees of freedom in each mode shape. This means that the centre of mass offset causes coupling between the degrees of freedom of the system. Although the amplitude of the excited degrees of freedom are relatively small, the greater values of $b x_{\alpha}$ result in the greater amplitudes. However, $b x_{\alpha}$ values greater than $10 \%$ are rare in wind turbine designs.

At the end of this section, it is worth commenting on the amplitude of the spanwise degrees of freedom shown in Fig.2.6.c. Note that the zero amplitude portion of the spanwise mode shape corresponds to the portion of the blade which has straight shape, and accordingly, the non-zero amplitude belongs to the circular portion of the approximate troposkien blade. A true troposkien shape would guarantees a smooth transition in the amplitude of this mode shape.

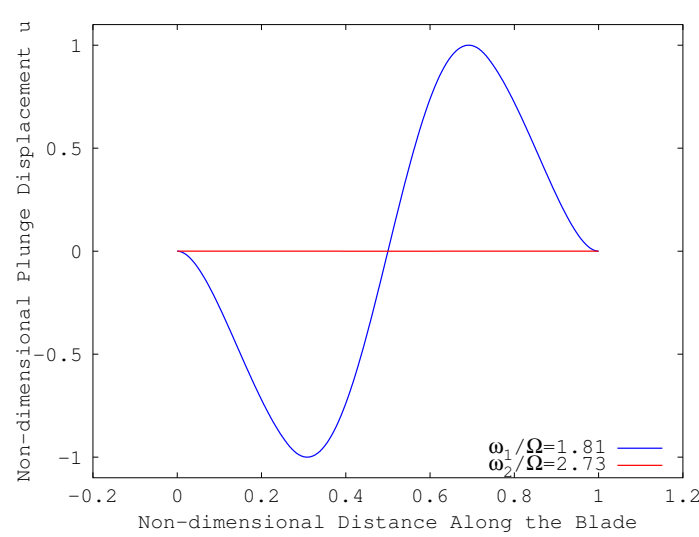

(a)

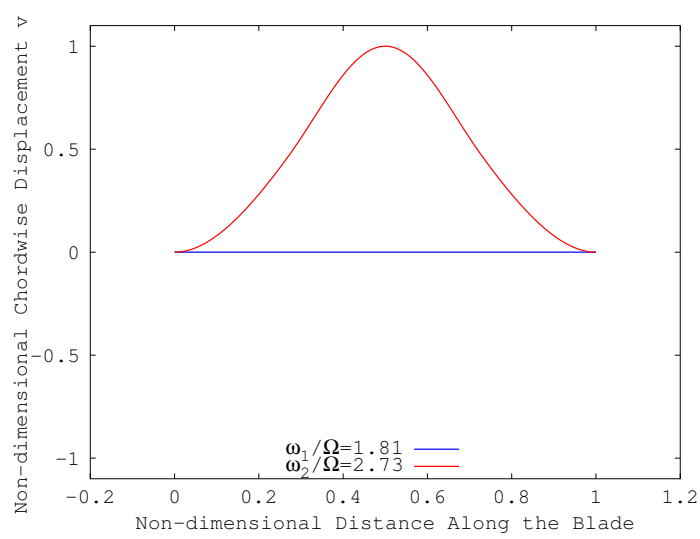

(b) 


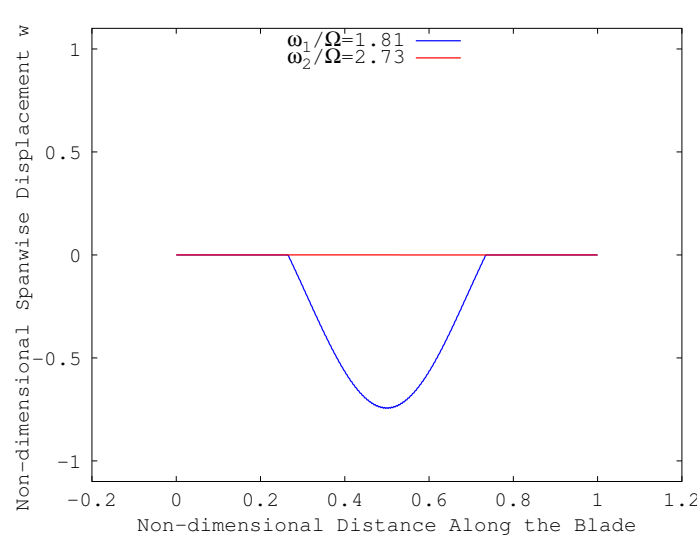

(c)

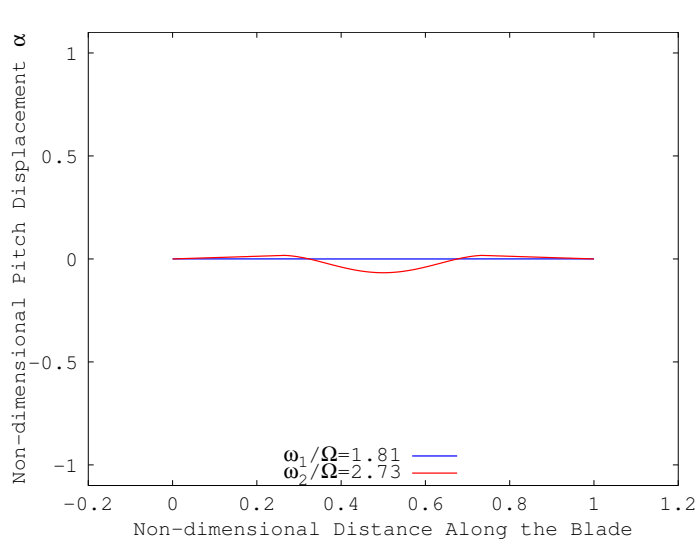

(d)

Figure 2.6: First two mode shapes: angular velocity $\Omega=50.6 \mathrm{rpm}$, no centre of mass offset ( $x_{\alpha}=0$ ), Coriolis effect excluded. (a) Plunge displacement (b) Chordwise displacement (c) Spanwise displacement (d) Pitch displacement

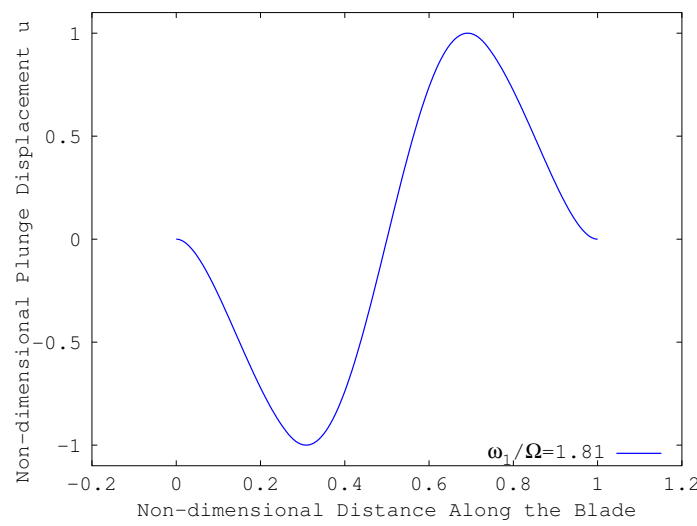

(a-1)

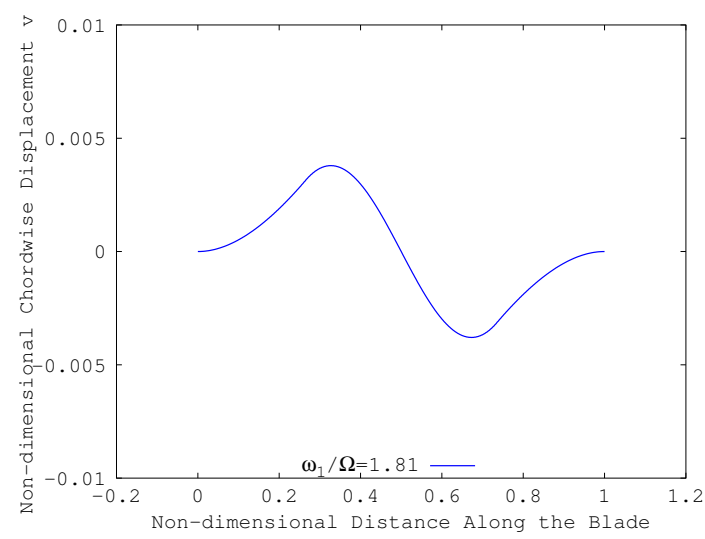

(b-1)

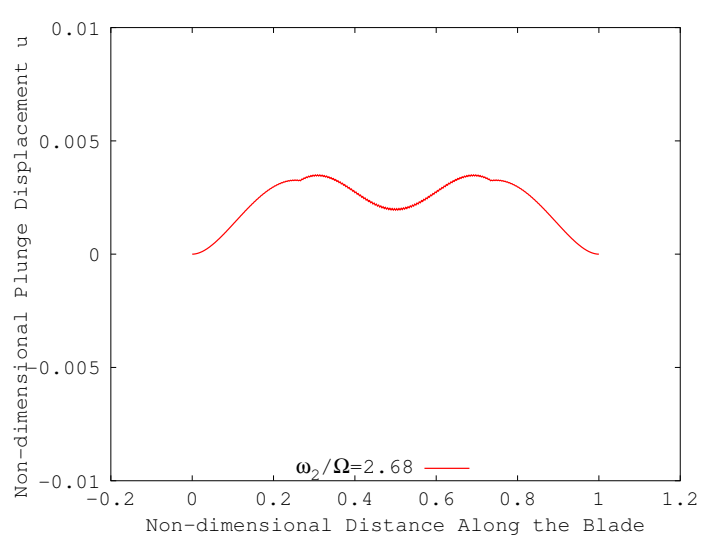

(a-2)

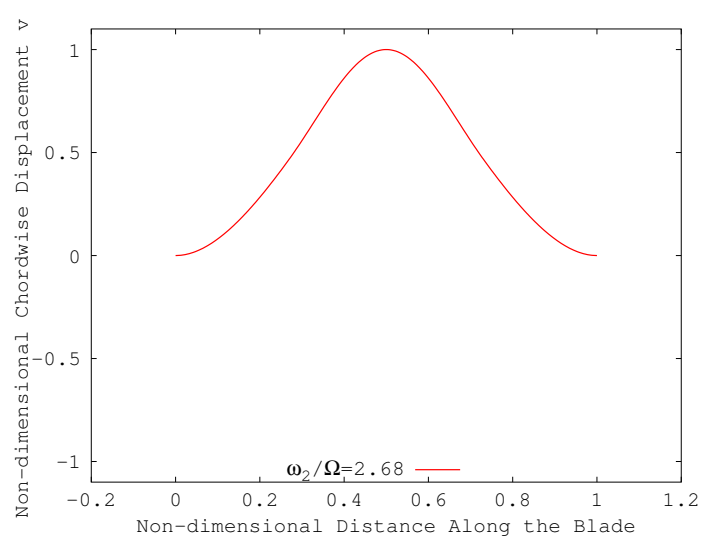

(b-2) 


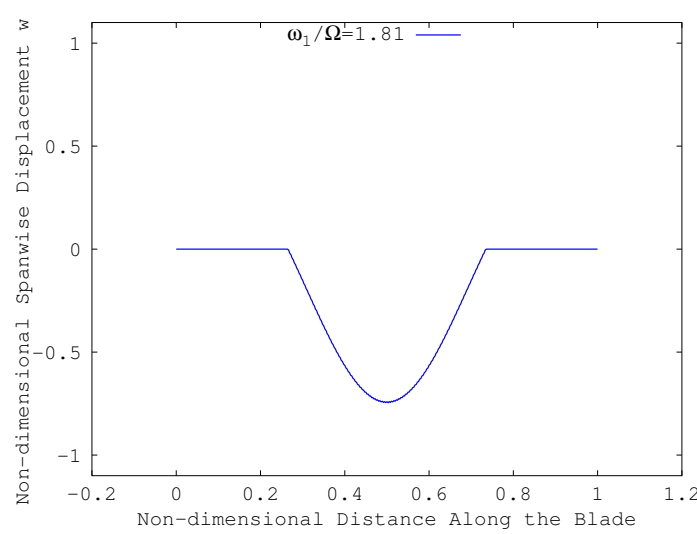

(c-1)

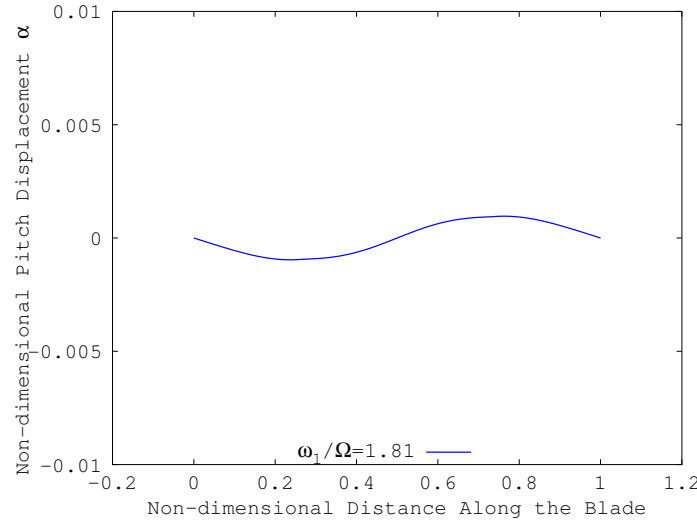

$(d-1)$

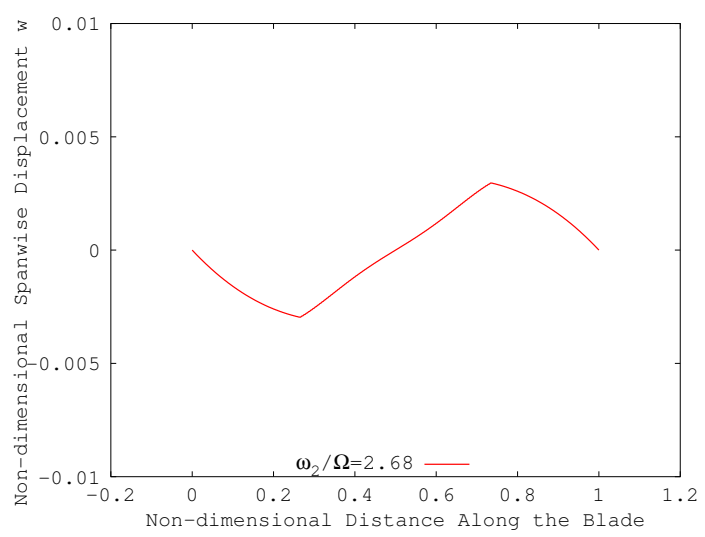

(c-2)

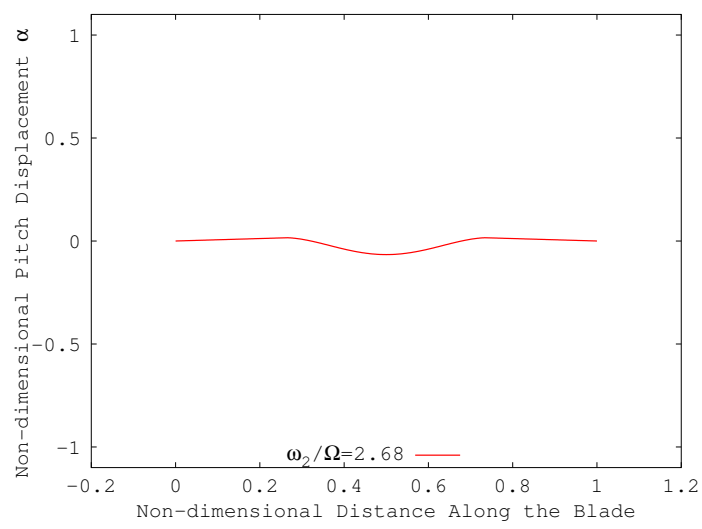

$(d-2)$

Figure 2.7: First two mode shapes: angular velocity $\Omega=50.6 \mathrm{rpm}, 10 \%$ centre of mass aft ( $x_{\alpha}=-0.1$ ), Coriolis effect excluded. (a) Plunge displacement (b) Chordwise displacement (c) Spanwise displacement (d) Pitch displacement 


\subsubsection{Coriolis Force Included}

The structural dynamic equation (Eq. 2.231$)$ used in this study is expressed in a rotational frame of reference. This form of representation introduces two terms to the equation that do not appear explicitly if the equation is derived in a stationary frame of reference. These two terms are the displacement dependent matrix $K_{D}$ and the velocity dependent matrix $C$. The role that the matrix $K_{D}$ (the softening stiffness matrix) plays in destabilizing the system is already discussed in the previous section. Here, the focus is on the study of the matrix $C$, which accounts for the Coriolis forces.

In order to elucidate the effect of the Coriolis forces, the complete form of Eq. $\overline{2.23}$, which involves the matrix $C$, is analyzed. The following statements summarize the findings from the eigenvalue analysis performed here:

- Since the Coriolis forces do not introduce any damping mechanism to the system, the system still remains conservative. Hence, the eigenvalues remain as pure imaginary quantities, representing the natural frequencies of the system. The magnitude of the natural frequencies changes slightly when compared with the case that the Coriolis forces were excluded.

- The eigenvectors associated with different degrees of freedom are either pure imaginary or pure real quantities. This property of the eigenvectors is dictated by the skew-symmetric nature of the Coriolis matrix $C$. It is observed that due to the Coriolis forces, $90^{\circ}$ phase shift between certain degrees of freedom kicks in. This phase shift results in a combination of pure real and pure imaginary eigenvectors.

- The Coriolis forces couple certain degrees of freedom. Fig.2.8 shows the first and second mode shapes when the Coriolis forces are included and the centre of mass offset is considered to be zero. As illustrated in Fig.2.8.b and Fig.2.8.d, the chordwise 
and pitch displacements, which were absent previously, are now excited in the first mode shape. Similarly, the plunge and spanwise displacement modes are now present in the second mode shape.

- In comparison with the centre of mass offset, the extent of coupling is greater. This fact can be seen in the amplitude of the Coriolis-excited degrees of freedom.

- The Coriolis forces originate phase shifts between certain degrees of freedom. Table 2.$]$ contains the phase information for the first and second mode shapes. The reason behind the phase shift could be explained as follows: the Coriolis effect is a velocity-dependent phenomenon. Moreover, velocity always lead the displacement by $90^{\circ}$ phase shift. Therefore, when the Coriolis forces excite a particular degree of freedom, that degree will be in a $90^{\circ}$ phase shift with the other degrees of freedom. For instance, in the first mode shape, the Coriolis effect gives rise to the seen chordwise and pitch components of the mode shape. As a result, these degrees of freedom are correspondingly in a $90^{\circ}$ phase shift with respect to the plunge and spanwise components of the mode shape. Using an analogous argument, one can deduce that the plunge and spanwise components of the second mode shape must also have a $90^{\circ}$ phase shift with respect to the chordwise and pitch components, as verified in the analysis.

\begin{tabular}{|c|c|c|c|c|}
\hline Mode shape & Plunge $(u)$ & Chordwise $(v)$ & Spanwise $(w)$ & Pitch $(\alpha)$ \\
\hline $1^{\text {st }}$ & $0^{\circ}$ & $90^{\circ}$ & $0^{\circ}$ & $90^{\circ}$ \\
\hline $2^{\text {nd }}$ & $90^{\circ}$ & $0^{\circ}$ & $90^{\circ}$ & $0^{\circ}$ \\
\hline
\end{tabular}

Table 2.1: Phase information of the first and second mode shapes 


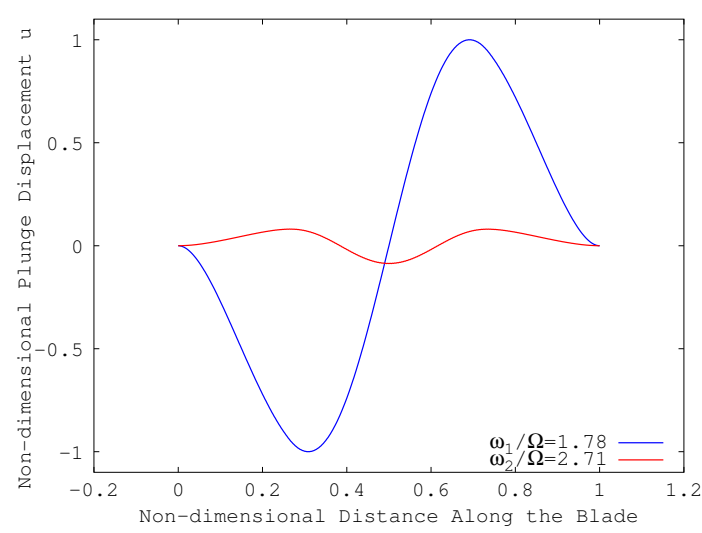

(a)

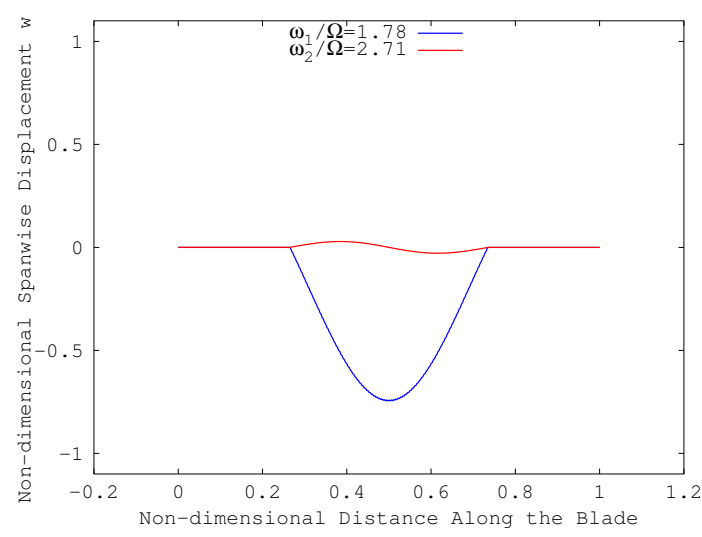

(c)

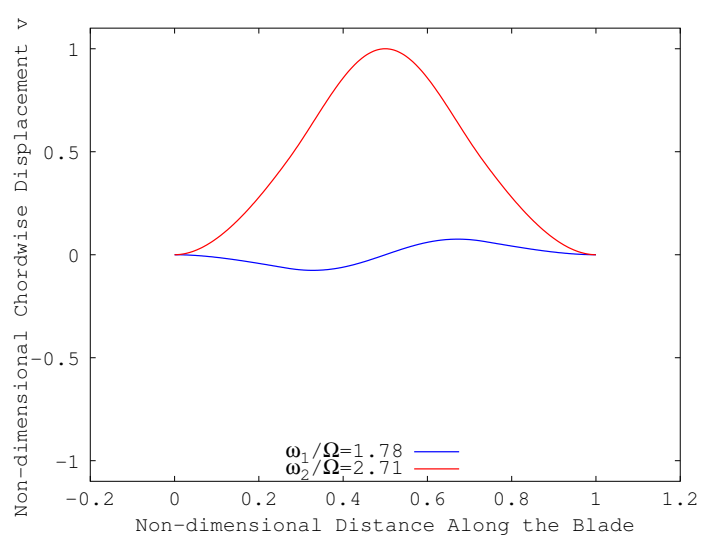

(b)

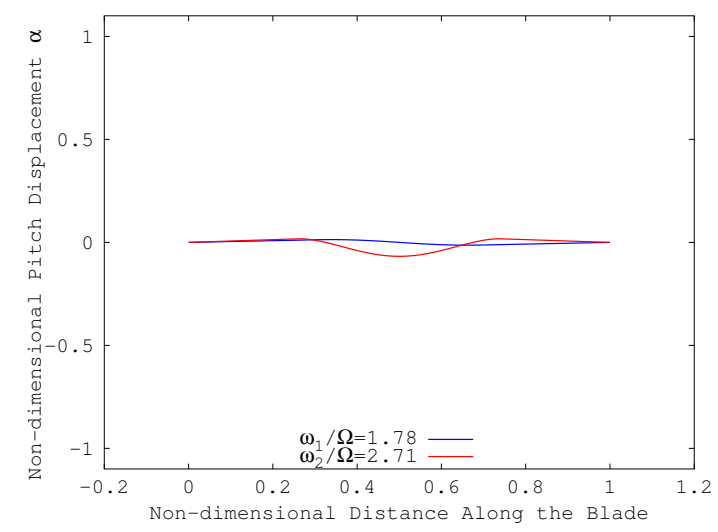

(d)

Figure 2.8: First two mode shapes: angular velocity $\Omega=50.6 \mathrm{rpm}$, no centre of mass offset $\left(x_{\alpha}=0\right.$ ), Coriolis effect included. (a) Plunge displacement (b) Chordwise displacement (c) Spanwise displacement (d) Pitch displacement 
This chapter is concluded by demonstrating the fan plot of the 17-meter DOE-Sandia VAWT, depicted in Fig.2.9. The graph shows the variation of the first three natural frequencies with respect to the angular velocity of the wind turbine. The first three harmonics (1P, 2P, 3P) and the operating rpm of the wind turbine (50.6) are also illustrated. The harmonics basically present the first three fundamental frequencies of the external loading. This graph is extremely important when one studies the resonance related issues of the VAWTs. The intersection of the operating rpm, the vertical line at $50.6 \mathrm{rpm}$, with the natural frequency curves, reveals the natural frequencies of the system at $50.6 \mathrm{rpm}$. Any harmonics that passes through or close by this intersection could cause resonance or high amplitude dynamic response. Hence, in the design process of the wind turbines, these intersections have to be avoided. From Fig.2.9, one could infer that the 17-meter DOE-Sandia VAWT does not experience major resonance related problems.

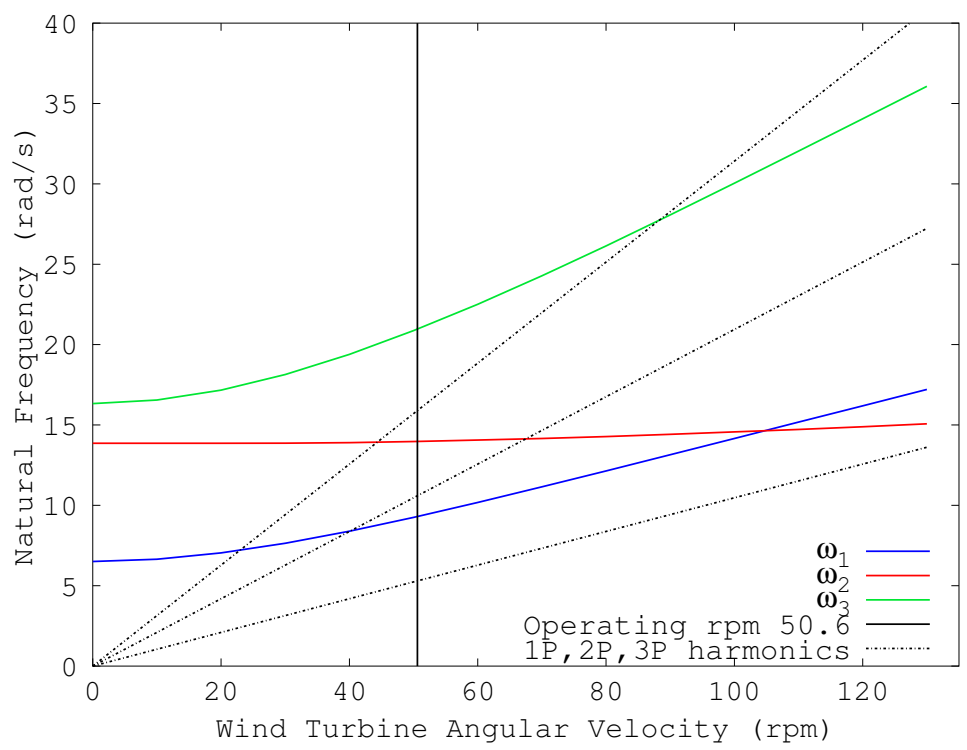

Figure 2.9: The fan plot of the DOE-Sandia 17-meter Darrieus vertical axis wind turbine: operating angular velocity $\Omega=50.6 \mathrm{rpm}, 10 \%$ centre of mass aft ( $\left.x_{\alpha}=-0.1\right)$, Coriolis effect included. 


\subsection{Conclusions}

A mixed finite element formulation is developed for the structural dynamic analysis of a Darrieus type VAWTs with the troposkien shape. The modal analysis shows that the natural frequencies of the system rise with the increase of the angular velocity of the turbine. This property is clearly shown to be due to the axial stresses along the blade caused by the centrifugal forces. It is also outlined that the centre of mass offset as well as the Coriolis forces couple the degrees of freedom. The coupling induced by the Coriolis forces are also accompanied by the phase shifts between the degrees of freedom. Finally, a fan plot is produced for the 17-meter DOE-Sandia VAWTs, which emphasizes the resonance-free operating range of the wind turbine rpm. 


\section{Chapter 3}

\section{Aerodynamic Analysis}

\subsection{Introduction}

This chapter is devoted to describing the aerodynamic model used in this analysis. In this model, vortex filaments, which freely translate, rotate and stretch, represent the wake of the wind turbine. The effect of the wake on the blade is taken into account by updating the incident velocities at different airfoil sections along the blade. Using these velocities, the aerodynamic loading is obtained from airfoil experimental data tables. This already existing model is improved by: (1) incorporating the variable Reynolds number and (2) implementing parallel computation capability.

First, the theory behind the free vortex model is explained. Then, the implemented methodology is compared with the available numerical results to verify the validity of the implementation. Next, the spatial and temporal convergence analysis is conducted to evaluate the sensitivity of the method to the number of elements as well as the number of time steps. Finally, the scalability of the parallel implementation is discussed. 


\subsection{Free Vortex Model}

The following section briefly explains the underlying concepts of the free vortex model proposed by Strickland [Q]. For further details, one could refer to the reference [Q].

\subsubsection{Coordinate System}

The first step towards implementing the free vortex method involves dividing the blades into a number of spanwise elements. To accommodate the definition of these elements, a local coordinate system is established. The origin of this local coordinate system is located at the aerodynamic centre (AC) of the section of the blade. This coordinate system, which is illustrated in Fig.B.. a, is defined as : $\mathrm{s}$ is aligned along the span, points from the start node to the end node of the element, $\mathbf{c}$ is the spanwise vector directed towards the trailing edge, and $\mathbf{n}$ is constructed from the cross product of the other two axes, as follows

$$
\begin{aligned}
& \mathbf{s}=\frac{\left(X_{i+1}-X_{i}\right) \mathbf{i}+\left(Y_{i+1}-Y_{i}\right) \mathbf{j}+\left(Z_{i+1}-Z_{i}\right) \mathbf{k}}{\sqrt{\left(X_{i+1}-X_{i}\right)^{2}+\left(Y_{i+1}-Y_{i}\right)^{2}+\left(Z_{i+1}-Z_{i}\right)^{2}}}, \\
& \mathbf{c}=\cos \theta_{B} \mathbf{i}-\sin \theta_{B} \mathbf{k}, \\
& \mathbf{n}=\mathbf{s} \times \mathbf{c}
\end{aligned}
$$

where $\langle X, Y, Z\rangle$ is the global coordinates of the $\mathrm{AC}$ of any section of the blades, and $\theta_{B}$ is the blade azimuth angle. The global coordinates of the ACs on the blades and the movements of the vortices are tracked with respect to a global coordinate system, which is placed at the bottom of the wind turbine as shown in Fig. B.D.b. The coordinate of the ACs 
on the blade are determined by the following equations

$$
\begin{aligned}
\theta_{B} & =\frac{2 \pi}{N B}(B N-1)+\theta, \\
X & =-r \sin \theta_{B}, \\
Z & =-r \cos \theta_{B},
\end{aligned}
$$

where $r$ is the radius with respect to the vertical axis, $N B$ is the number of blades, $B N$ is the blade number and $\theta$ is the angle shown in Fig. J.b. Since in this chapter, it is assumed that the blades are rigid, the aerodynamic centres of the sections of the blade follow exactly the perimeter of a circle with radius $r$ in one revolution. As a result of this assumption, the $Y$ coordinates of the ACs on the blades are unchanged during the rotation, and can be obtained from the troposkien shape described in Section 2.2.

\subsubsection{Element Bound Vorticity}

The second step of this procedure deals with calculating the bound vortex strength associated to each blade element. To perform this, the velocity at each end of a blade element is evaluated by summing the blade rotational velocity $U_{t}$, free stream velocity $U_{\infty}$ and the induced velocity caused by the vortices $\langle U, V, W\rangle$, i.e.,

$$
\mathbf{U}_{\mathbf{R}}=\left(U+U_{\infty}+U_{t} \cos \theta_{B}\right) \mathbf{i}+V \mathbf{j}+\left(W-U_{t} \sin \theta_{B}\right) \mathbf{k}
$$

where $U_{t}=r \Omega$ and $\Omega$ is the turbine's angular velocity. The velocity associated to each blade element is considered to be the average of the velocities at both ends. Then, the 


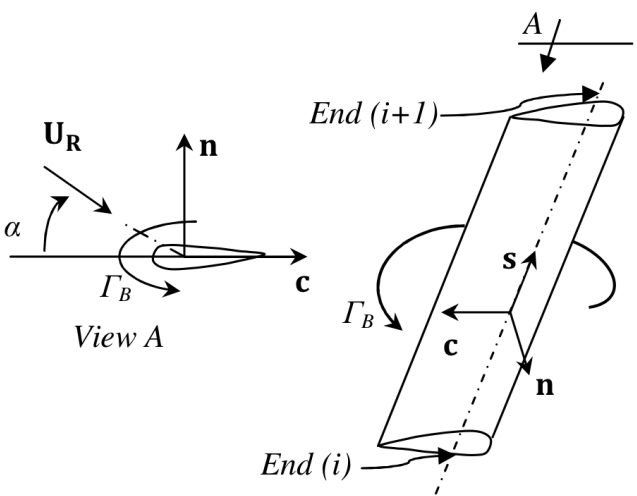

(a)

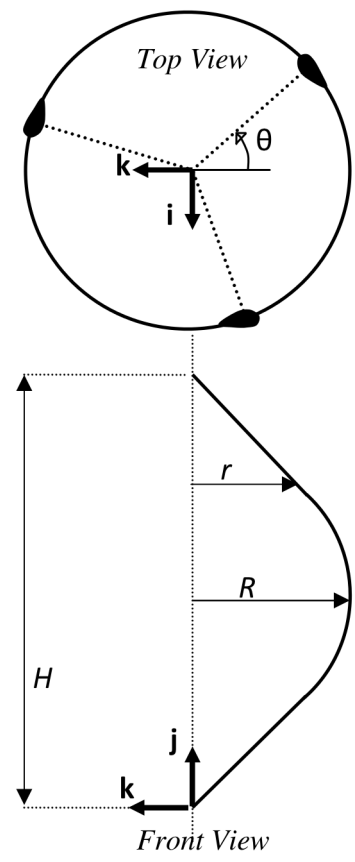

(b)

Figure 3.1: Free vortex model conventions (a) Blade element local coordinate system (b) Global coordinate system

Reynolds number and the angle of attack are evaluated as

$$
\begin{aligned}
R e & =\frac{\sqrt{\left(\mathbf{U}_{\mathbf{R}} \cdot \mathbf{n}\right)^{2}+\left(\mathbf{U}_{\mathbf{R}} \cdot \mathbf{c}\right)^{2}} C}{\nu}, \\
\tan \alpha & =-\frac{\mathbf{U}_{\mathbf{R}} \cdot \mathbf{n}}{\mathbf{U}_{\mathbf{R} \cdot \mathbf{c}}},
\end{aligned}
$$

where $C$ is the chord length and $\nu$ is the air kinematic viscosity. Using the value of Reynolds number and angle of attack calculated for each blade element, the lift $\left(C_{L}\right)$ and drag $\left(C_{D}\right)$ coefficients are obtained by interpolating the experimental data reported by Sheldahl and Klimas [25]. In their report, the lift and drag coefficients are extrapolated from the experimental data for six NACA symmetric airfoils, i.e., NACA009, NACA0012, 
NACA0015, NACA0018, NACA0021 and NACA0025. This information is provided for the Reynolds number ranges from $10^{4}$ to $10^{7}$, and the angle of attack spans from $0^{\circ}$ to $180^{\circ}$. The tangential force coefficient $C_{T}$ along the chord and the normal force coefficient $C_{N}$ normal to the chord are given as

$$
\begin{gathered}
C_{T}=C_{L} \sin \alpha-C_{D} \cos \alpha, \\
C_{N}=-C_{L} \cos \alpha-C_{D} \sin \alpha .
\end{gathered}
$$

Note that based on the above equations, $C_{T}$ is in the opposite direction of c, i.e., in the direction of the motion from the trailing edge to the leading edge, and $C_{N}$ is in the direction of the unit vector $\mathbf{n}$. Finally, the strength of the bound vortex per span $\Gamma_{B}$, which replaces each blade element, can be expressed in terms of the lift coefficient using the Kutta-Joukowski theorem as follows

$$
\Gamma_{B}=\frac{1}{2} C_{L} C U_{R}
$$

\subsubsection{Vortex Shedding}

Calculating the strength of the shed vortices, including the spanwise and trailing vortices, is the next step. The vortices are shed in the form of vortex filaments: spanwise filaments parallel to the span of the blade element and trailing tip filaments along the chord at both ends of the blade element. The strength of these filaments are computed according to the Kelvin and Helmholtz theorems. Based on the Kelvin's circulation theorem, the bound vortex strength around a contour enclosing the airfoil and its wake being zero before the 
start of the motion, must remain zero, i.e.,

$$
\frac{d \Gamma_{B}}{d t}=0
$$

Due to the fact that the direction of the blade element velocity changes constantly during one revolution, and the direction of the wind is fixed, the angle of attack that each blade element experiences changes periodically. Consequently, the amount of the generated lift and the bound vortex strength changes accordingly. This change in the bound vortex strength must be counteracted by a shed spanwise vortex, keeping the change of the total circulation equal to zero. This means

$$
\Gamma_{S}=\Gamma_{B_{t}}-\Gamma_{B_{t+\Delta t}}
$$

Fig.3.2 shows the shed spanwise vortex filament from a single blade element over the time. This figure also shows the trailing tip vortices at both ends of the element. Dictated by the Helmholtz theorem, the strength of these trailing tip vortex filaments, at both ends of the element, is equal to the strength of the bound vortex representing each blade element. Hence the difference between the strength of the bound vortices associated to each blade element, results in the trailing shed vortices that their strength are given as

$$
\Gamma_{T}=\Gamma_{B_{i+1}}-\Gamma_{B_{i}}
$$

where $\Gamma_{B_{i}}$ represents the bound vortex strength assigned to the blade element $i$. Note that at the sections where two blade elements join, these tip vortices do not exist physically; however, they have to be present numerically to represent the change of the bound vortex strength along the blade. 


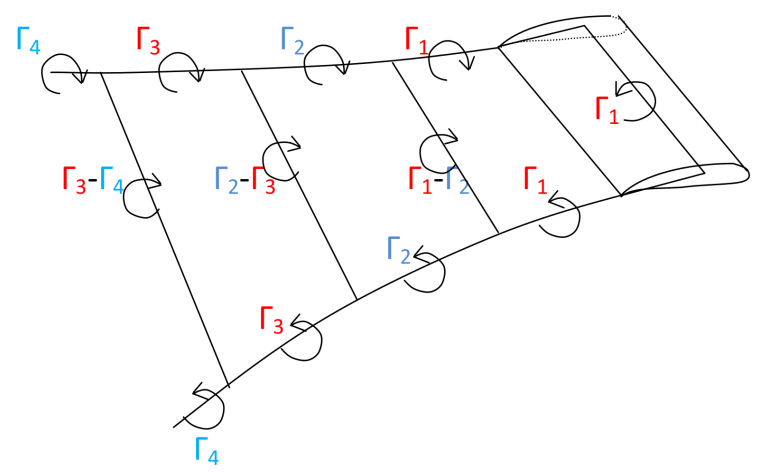

Figure 3.2: Vortex shedding for a single blade element

\subsubsection{Wake Convection}

Finally, the last step concerns with the calculation of the distance any point in the fluid domain travels during the time increment $\Delta t$. Among these points are the start and end points of the filaments. They convect by the local velocity at that point. An explicit time integration technique is used to calculate the travelled distance, expressed as

$$
\begin{aligned}
& \Delta x=\left(1.5 U_{t}-0.5 U_{t-\Delta t}+U_{\infty}\right) \Delta t, \\
& \Delta y=\left(1.5 V_{t}-0.5 V_{t-\Delta t}\right) \Delta t, \\
& \Delta z=\left(1.5 W_{t}-0.5 W_{t-\Delta t}\right) \Delta t,
\end{aligned}
$$

where, similar to Eq. B.3, $U, V$ and $W$ are the components of the induced velocity in $x, y$ and $z$ directions. The distance travelled by the vortices that just leave the blade, in their first time increment, can be calculated as

$$
\begin{aligned}
& \Delta x=\left(U_{t}+U_{\infty}\right) \Delta t, \\
& \Delta y=V_{t} \Delta t \\
& \Delta z=W_{t} \Delta t .
\end{aligned}
$$




\subsubsection{Remarks}

In order to complete the aforementioned discussion regarding the free vortex model, it is worth explaining the approach used for calculating the velocity induced by the vortex filaments. Moreover, some definitions related to the torque and power of the wind turbine are presented in the subsequent section.

\section{Induced Velocity}

The velocity induced by a vortex filament $\langle U, V, W\rangle$ including bound, spanwise and trailing vortices, with the strength $\Gamma$ used in Eq. 3.3 , is calculated using the Biot-Savart law given as

$$
\langle U, V, W\rangle=\frac{\Gamma}{4 \pi} \int_{l} \frac{\mathbf{r} \times \mathbf{d} \mathbf{l}}{r^{3}},
$$

where $\mathrm{dl}$ is the incremental length along the vortex filament and $\mathbf{r}$ is the position vector from the incremental length dl to the desired location in the fluid domain. If it is assumed that the vortex filaments are straight lines, one obtains a more convenient form of the above equation represented as

$$
\langle U, V, W\rangle=\frac{\Gamma}{4 \pi} \frac{\mathbf{c} \times \mathbf{a}}{|\mathbf{c} \times \mathbf{a}|^{2}}\left(\frac{\mathbf{a} \cdot \mathbf{b}}{b}-\frac{\mathbf{a} \cdot \mathbf{c}}{c}\right) .
$$

As shown in Fig. B.3, a extends from the first node to the second node of the vortex filament, $\mathbf{b}$ and $\mathbf{c}$ are the vectors that start from the location of interest in the domain and end at the second and first nodes of the vortex filament respectively. 


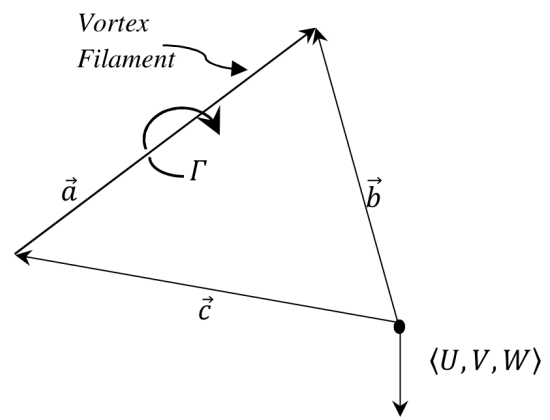

Figure 3.3: Induced velocity at a point by vortex filament

\section{Rotor Performance}

In the preceded sections, it was explained how to obtain the tangential $\left(C_{T}\right)$ and normal $\left(C_{N}\right)$ force coefficients. One can easily convert these coefficients to the actual amount of tangential and normal forces as given by

$$
\begin{aligned}
& F_{T}=\frac{1}{2} \rho C l_{e} C_{T} U_{R}^{2}, \\
& F_{N}=\frac{1}{2} \rho C l_{e} C_{N} U_{R}^{2},
\end{aligned}
$$

where $l_{e}$ is the length of the blade element. Note that the generated torque can be calculated as $Q=r F_{T}$ and the generated power as $P=\Omega Q$. Therefore, the non-dimensional torque $\left(C_{Q}\right)$ and power $\left(C_{P}\right)$ coefficients for a single blade is obtained as the sum of these quantities over the total number of the elements of the blade $N E$, given as

$$
\begin{aligned}
C_{Q} & =\sum_{1}^{N E} \frac{r F_{T}}{\frac{1}{2} \rho R A_{F} U_{\infty}^{2}}, \\
C_{P} & =\sum_{1}^{N E} \frac{\Omega Q}{\frac{1}{2} \rho A_{F} U_{\infty}^{3}},
\end{aligned}
$$


where $A_{F}$ is the rotor swept area. It is also common to average the power over one revolution and define $\bar{C}_{P}$ as

$$
\bar{C}_{P}=\frac{1}{N T I} \sum_{1}^{N T I} C_{P}
$$

where NTI is the number of time increments per revolution of the rotor.

\subsection{Numerical Validation}

As mentioned earlier, Strickland et al. [9] formulated the aforementioned free vortex model, and then based upon that, they developed the VDART3 ( Vortex method of solution for the DARrieus Turbine in 3 dimensions) software for the aerodynamic analysis of VAWTs. The VDART3 code takes non-dimensional parameters as inputs, such parameters

are the chord to radius ratio $\frac{C}{R}$, the height to radius ratio $\frac{H}{R}$, the tip speed ratio $\frac{R \Omega}{U_{\infty}}$, and the lift and drag coefficients for only one Reynolds number.

In the current investigation, a FORTRAN 90 based software called FEM-Vort is developed for the aeroelastic analysis of a VAWTs with troposkien shape. The elastic part of the software is coded using the formulation presented in Chapter 2, and the aerodynamic part is based on the Strickland free vortex model. Unlike VDART3, FEM-Vort is implemented based on dimensional input parameters. The reason behind this choice is explained later in this chapter. Here, to verify the validity of the aerodynamic part of FEM-Vort, the results generated by this software are compared with the ones reported by Strickland et al. [9] in the original Sandia report where VDART3 is explained in detail. For the purpose of this validation, the elastic part of FEM-Vort software is switched off.

The original Sandia report [0] demonstrates the normal and tangential forces generated at the equator of a two-bladed wind turbine with the following specifications: a chord 
to equator ratio of $\frac{C}{R}=0.135$, a height to radius ratio of $\frac{H}{R}=2$, a tip speed ratio of $\frac{R \Omega}{U_{\infty}}=5$, and 5 elements per blade $(N E=5)$. The lift and drag coefficients used are the ones for NACA0012 airfoil at the Reynolds number of $0.3 \times 10^{6}$. The corresponding input parameters used in FEM-Vort are as follows: height $H=2 m$, equator radius $R=1 \mathrm{~m}$, chord length $C=0.135 \mathrm{~m}$, the wind turbine angular velocity $\Omega=45 \mathrm{rad} / \mathrm{s}$, and the wind velocity $U_{\infty}=9 \mathrm{~m} / \mathrm{s}$. The same lift and drag data for the Reynolds number of $0.3 \times 10^{6}$ is also incorporated. This comparison is illustrated in Fig. 3.4.a. The parameters $F_{T}^{+}$and $F_{N}^{+}$ in this figure, which are proportional to the tangential and normal forces, are defined as

$$
F_{T}^{+}=C_{T}\left(\frac{U_{R}}{U_{\infty}}\right)^{2} \quad \text { and } \quad F_{N}^{+}=C_{N}\left(\frac{U_{R}}{U_{\infty}}\right)^{2}
$$

Fig.3.4 shows an absolute agreement between the FEM-Vort and VDART3 predictions $\left(F_{T}^{+}\right.$ and $F_{N}^{+}$). This fulfils the objective of ensuring that the implementation of the free vortex model in FEM-Vort is error-free.

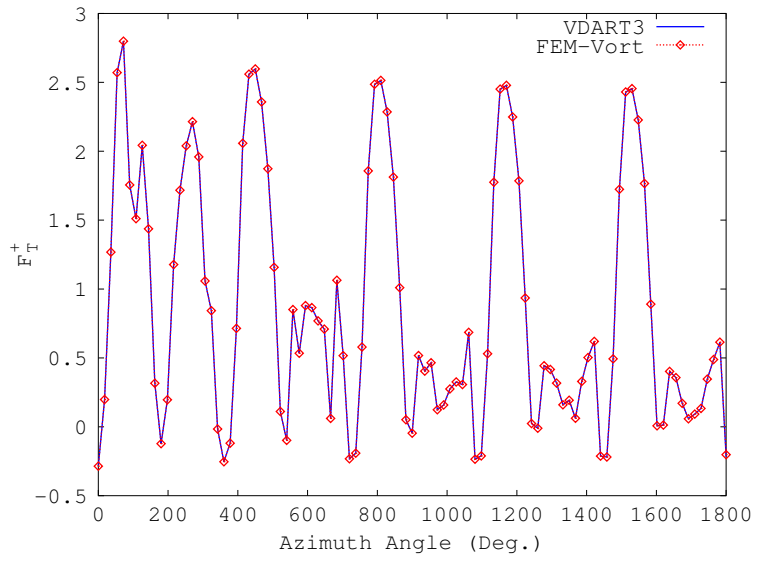

(a)

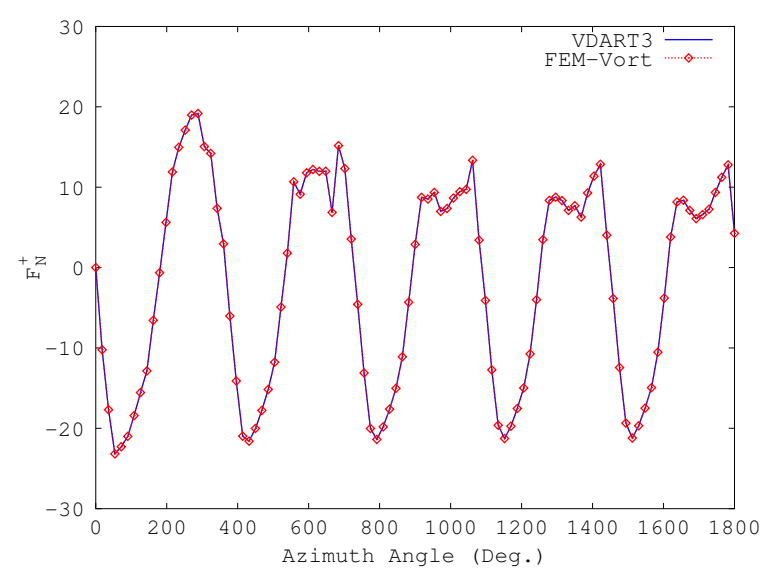

(b)

Figure 3.4: Comparison of equator forces between the FEM-Vort aerodynamic and VDART3: chord to equator ratio of $\frac{C}{R}=0.135$, height to radius ratio of $\frac{H}{R}=2$, tip speed ratio of $\frac{R \Omega}{U_{\infty}}=5$, FEM-Vort with fixed Reynolds number 
Due to the lack of a complete set of experimental data in 1980, Strickland et al. [Q] developed VDART3 such that the lift and drag coefficients for all the blade elements are interpolated using the experimental data at only one Reynolds number regime, the one that is close to the calculated Reynolds number of the equator of the wind turbine. Since there was no need to calculate the Reynolds number, the calculation of the absolute value of velocity was not required. This is the main reason that VDART3 was written in terms of the non-dimensional parameters mentioned earlier. However, this assumption is not a reasonable assumption due to the facts that

- Each blade element has a different radius from the vertical axis; hence, it has a different velocity from the other elements.

- The velocity of each blade element changes its direction with the free stream velocity during one revolution.

Therefore, the magnitude of the relative velocity as well as the Reynolds number changes not only from one element to the other but also from one position to another. Hence, in the light of extensive experimental data available now [25], FEM-Vort takes into consideration the variation of the Reynolds number. Consequently, for the sake of calculating the Reynolds number which demands the absolute value of velocity, FEM-Vort is written for the dimensional parameters.

Fig. [.5.5 shows the comparison between VDART3 and FEM-Vort when the variable Reynolds number effect is included in FEM-Vort. The two results exhibit significant differences when one compares the tangential force parameter $F_{T}^{+}$specially at the peaks. This discrepancy stems from the sensitivity of the lift coefficient to the variation of Reynolds number. To explore this, let us examine the three graphs presented in Fig.3.6. From Fig.3.6.a, which shows the variation of angle of attack during five revolutions obtained from FEM-Vort, one 
can observe that the maximum tangential force happens when the angle of attack is approximately $10^{\circ}$. This angle of attack corresponds to a Reynolds number at about $0.48 \times 10^{6}$, as shown in Fig.3.6.b. Comparing this Reynolds number with the one used in VDART3 $\left(0.3 \times 10^{6}\right)$ explains the disparity in the results of FEM-Vort and VDART3. This conclusion will become more evident when one inspects the variation of the lift coefficient with the Reynolds number in Fig.B.6.c. Implied in this figure, the lift coefficient at the angle of attack of $10^{\circ}$ is very sensitive to the value of the Reynolds number. Conclusively, the variation of the Reynolds number play an important role in an accurate prediction of the aerodynamic forces. This effect is taken into account in the development of FEM-Vort.

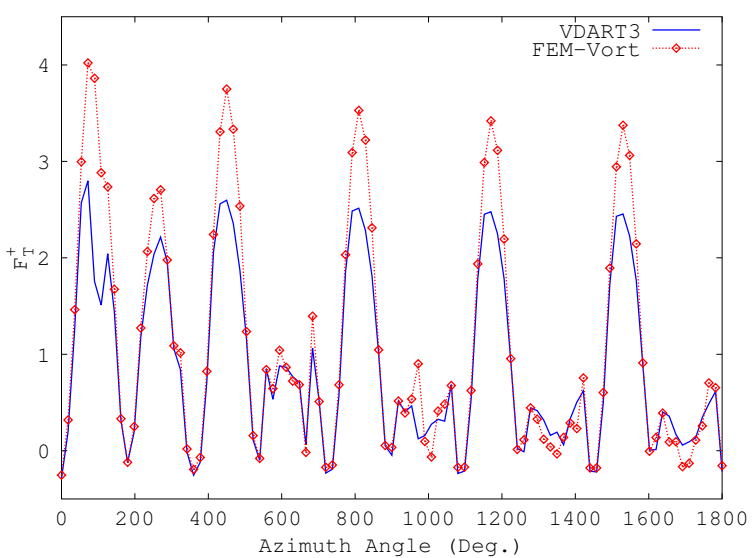

(a)

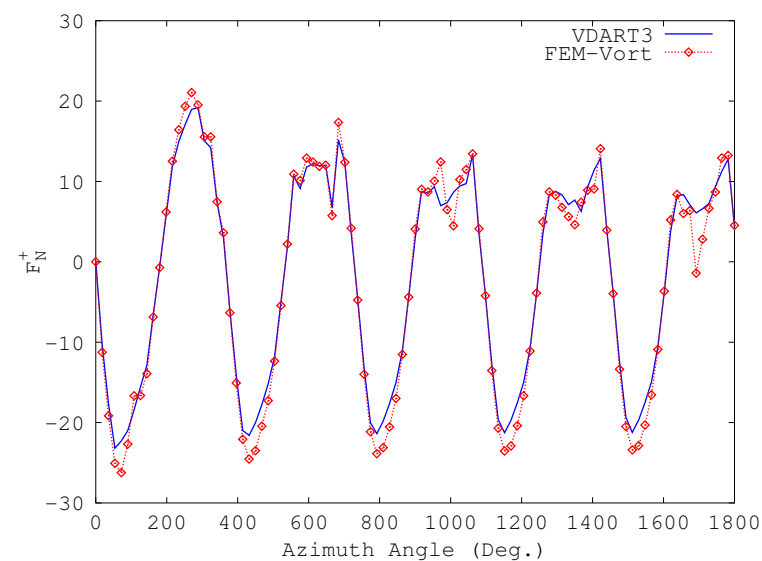

(b)

Figure 3.5: Comparison of equator forces between the FEM-Vort aerodynamic and VDART3: chord to equator ratio of $\frac{C}{R}=0.135$, height to radius ratio of $\frac{H}{R}=2$, tip speed ratio of $\frac{R \Omega}{U_{\infty}}=5$, FEM-Vort with variable 


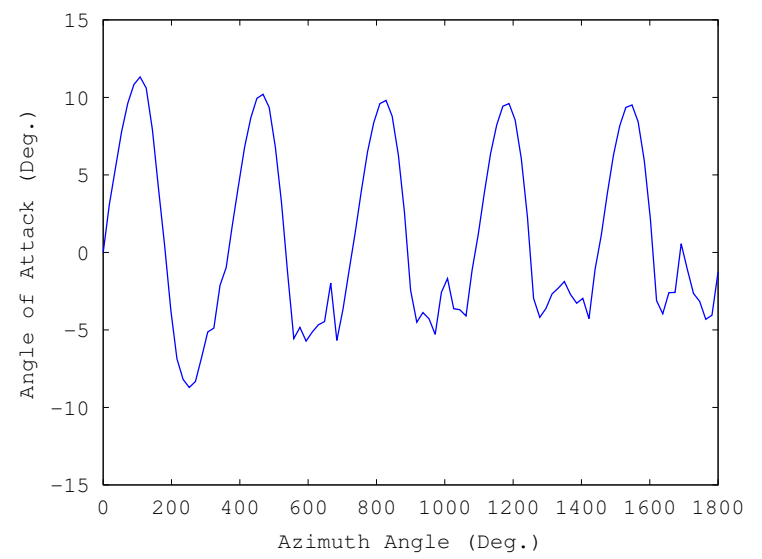

(a)

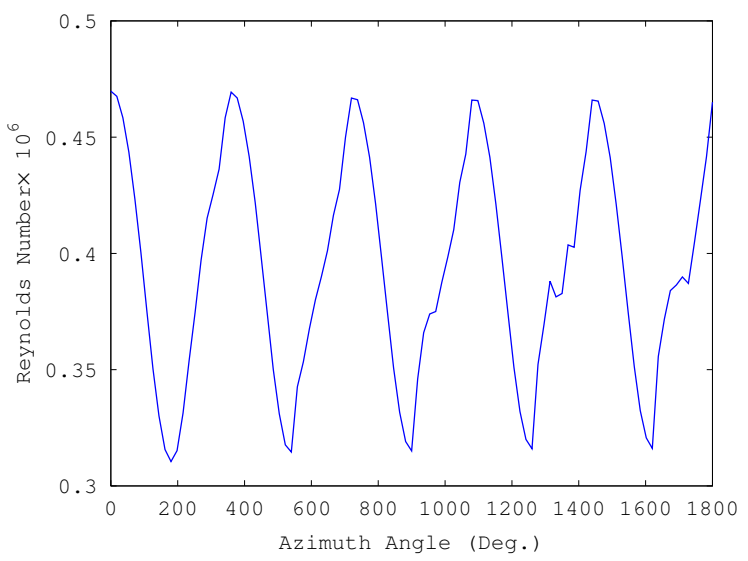

(b)

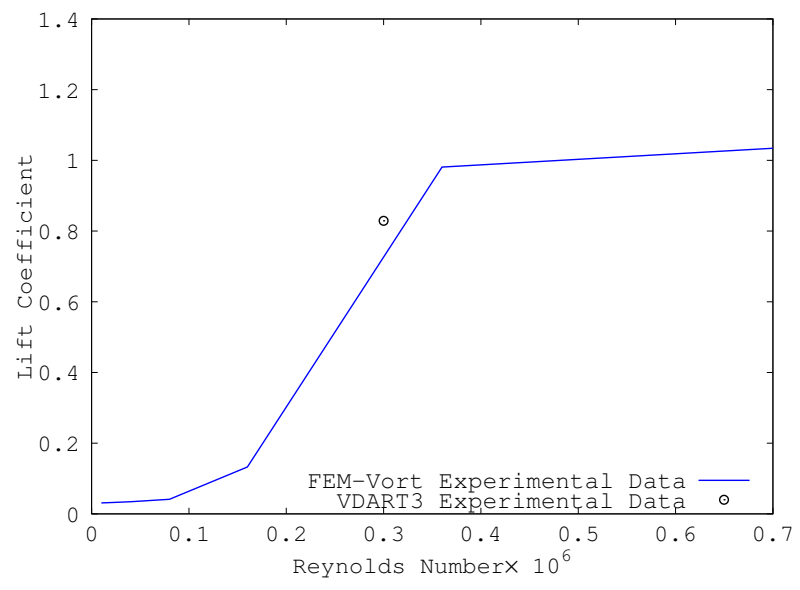

(c)

Figure 3.6: The FEM-Vort aerodynamic model: height $H=2 m$, equator radius $R=1 \mathrm{~m}$, chord length $C=0.135 \mathrm{~m}$, the wind turbine angular velocity $\Omega=45 \mathrm{rad} / \mathrm{s}$ and the wind velocity $U_{\infty}=9 \mathrm{~m} / \mathrm{s}$ (a) Variation of angle of attack (b) Variation of Reynolds number (c) Variation of lift coefficient at $A O A=10^{\circ}$ with Reynolds number 


\subsection{Convergence Analysis}

In numerical analysis, it is crucial to ensure that the numerical solution most accurately mimics the physics of the problem by minimizing the influence of the numerical artifacts. Examples of these numerical artifacts are the ones that might arise from spatial and temporal resolutions. Here, a spatial and temporal convergence analysis is carried out striving at studying the effect of the number of elements as well as the number of time steps. This analysis determines the suitable number of elements and time steps for the aeroelastic analysis which will be presented in the next chapter. The wind turbine chosen for this study is the 17-meter DOE-Sandia used in the previous chapter. One could refer to reference [26] for the specifications of this wind turbine.

\subsubsection{Spatial Convergence}

To ensure the autonomy of the numerical solution from the number of blade elements, the results obtained from different number of blade elements are compared. One anticipates that more number of elements leads to a more accurate solution at the expense of higher computer simulation time; hence, a compromise has to be reached. Fig.3.7.a shows the tangential force coefficient $C_{T}$ at the equator of the 17-meter DOE-Sandia VAWT with four different number of blade elements. The results obtained by 23 and 47 elements are almost identical. Therefore, considering the computer run time restriction, 23 blade elements are chosen for the rest of the analyses.

\subsubsection{Temporal Convergence}

Similar to the number of blade elements, a compromise between computer run time and accuracy has to be reached for selecting the suitable number of time steps. Another factor 
that plays a role is the fact that very small time step allows high frequency components to manifest in the aerodynamic forces. Some of these high frequency components not only do not increase the accuracy of the results but also result in spurious high frequency structural response.

Fig.B.7.b shows the tangential force coefficient $C_{T}$ at the equator for 20, 40 and 60 time steps per revolution. Evidently, all the results show the same mean trend. The only difference relies on the fact that 60 time steps introduce high frequency fluctuations in the solution. In order to decide on the number of time steps, it is insightful to inspect the Nyquist frequencies associated with each time step. According to the angular velocity of the wind turbine (50.6rpm), the Nyquist frequency corresponding to 20, 40 and 60 time steps are $53.25 \mathrm{rad} / \mathrm{s}, 106.49 \mathrm{rad} / \mathrm{s}$ and $159.74 \mathrm{rad} / \mathrm{s}$ respectively. These Nyquist frequencies cover 10, 20 and 30 harmonics. It seems that covering 10 harmonics could be enough to represent the aerodynamic forces. Hence, considering the computer run time restriction, 20 time steps per revolution is chosen for the rest of the analyses.

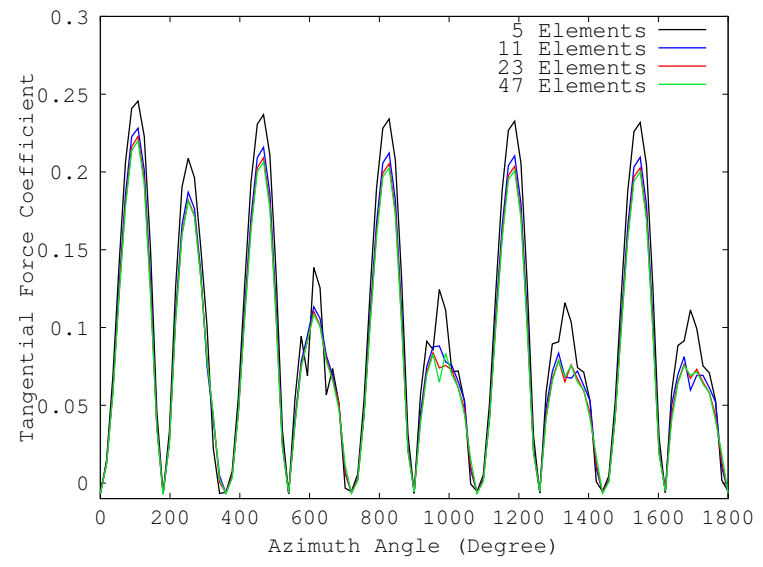

(a)

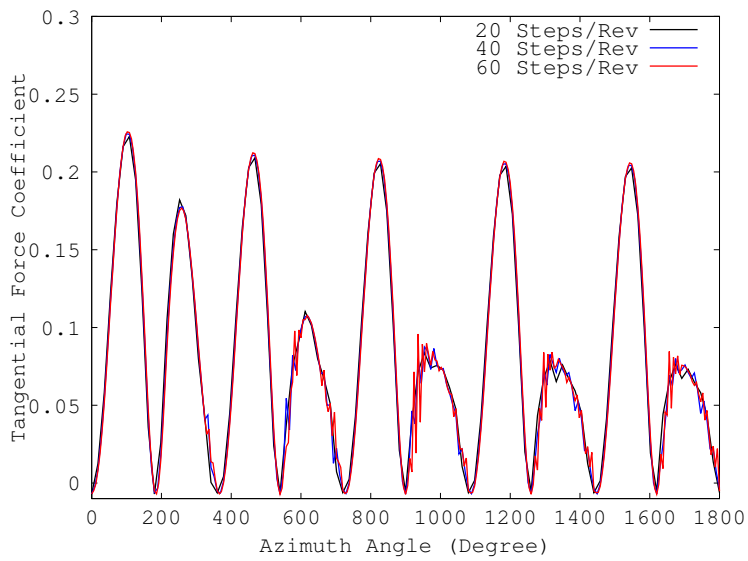

(b)

Figure 3.7: Convergence analysis: 17 meter DOE-Sandia VAWT, the wind turbine angular velocity $\Omega=50.6 \mathrm{rpm}$ and the wind velocity

$U_{\infty}=22 m p h$ (a) Number of blade elements (b) Number of time steps per revolution 


\subsection{Parallel Implementation}

The aerodynamic model presented here provides a more detailed representation of the wake compared to the momentum based models. This is a significant advantage specially when aeroelastic analysis demands a more accurate predictions of the aerodynamic forces. However, from a computational point of view, this model is costly. Although, the computer run time of this model is considerably less than a full CFD simulation, it is still more computationally expensive than the momentum based methods. This cost becomes more pronounced when parametric studies are conducted to investigate the influence of parameters such as geometry and blade shapes. To tackle such problem, a simple parallel version of the aforementioned model is implemented.

The parallelism implemented herein takes advantage of a shared memory parallel programming interface, the so-called OpenMP. The reason for this selection originates from the physical nature of the free vortex model: according to several computational runs performed in this study, on average, more than $90 \%$ of the computation time of this model is spent on calculating the vortex filament induced velocity at different points in the fluid domain. This includes the computation of the velocity at the aerodynamic centres of the blade sections as well as the start and end points of the other vortex filaments travelling freely in the wake. In a parallel computation framework, imagine assigning a cluster of vortex filaments named $i$ to the $i$ th CPU of the machine. For calculating the vortex induced velocity at the nodes of one vortex filament in cluster $i$, the effect of all other vortex filaments inside cluster $i$ and inside all other clusters have to be evaluated. This means that for calculating the induced velocity at one node of one vortex filament, CPU $i$ has to talk to other CPUs $n \times m$ times, where $n$ is the number of clusters and $m$ is the number of vortex filaments inside each cluster. This value has to be multiplied by $m$ when one considers the total number of cross talks for all the vortex filaments inside cluster $i$. This simple calculation 
shows how massive the cross talks between CPUs could be. This massive communication between CPUs easily justifies that the implementation of the current vortex model is not suitable for distributed-memory machines. On the contrary, this model lends itself well to the shared-memory implementation. This is mainly due to the fact that each CPU takes the information it requires from a shared memory, accessible to all the CPUs. By using this approach, the cross-talks between the CPUs are avoided.

The shared memory parallel implementation of the free vortex model is performed using OpenMP. The routine that calculates the vortex induced velocities consists of four for loops that are parallelized easily by utilizing the $\$ \$ O M P D O$ directive. A case is tested where the 17-meter DOE-Sandia VAWT completes 10 full revolutions with an angular velocity of $50.6 \mathrm{rpm}$. The blades are divided into 23 elements each and 40 time steps are used in each revolution. The numerical experiments are carried out on a SPARC64 VII machine that has four 2.4 GHz Quad Core processors with 32 GB RAM and 2 hardware threads on each core. To ensure that the parallelism is implemented correctly, different quantities obtained from the parallel implementation were compared against the results of the serial implementation. As an example of such comparisons, Fig. B.8 illustrates the tangential force coefficient obtained for the aforementioned case study using 24 CPUs. The exact match between the two sets of results reveals the validity of the parallel implementation. Furthermore, Fig.5.9.a shows the computer run time for different number of CPUs. The serial version takes about five hours to complete; however, as the number of CPUs increases the run time reduces. Fig. B.9.b shows the scalability graphs in terms of the speed up, where Speedup $=\frac{\text { serial run time }}{\text { parallel run time }}$. This graph shows that the parallel run scales quite well. The deviation from the linear scalability could be attributed to the unparallelized part of the code and hardware restrictions. 


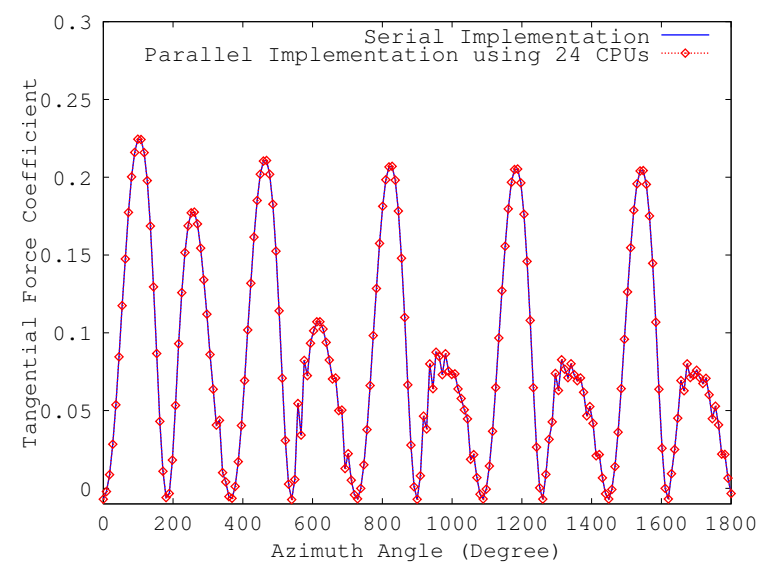

Figure 3.8: Verification of the parallel implementation in terms of the tangential force coefficient: comparison of the serial and the parallel (using 24 CPUs) implementations, 17 meter DOE-Sandia VAWT, 23 blade elements, 10 revolutions, 40 steps per revolution

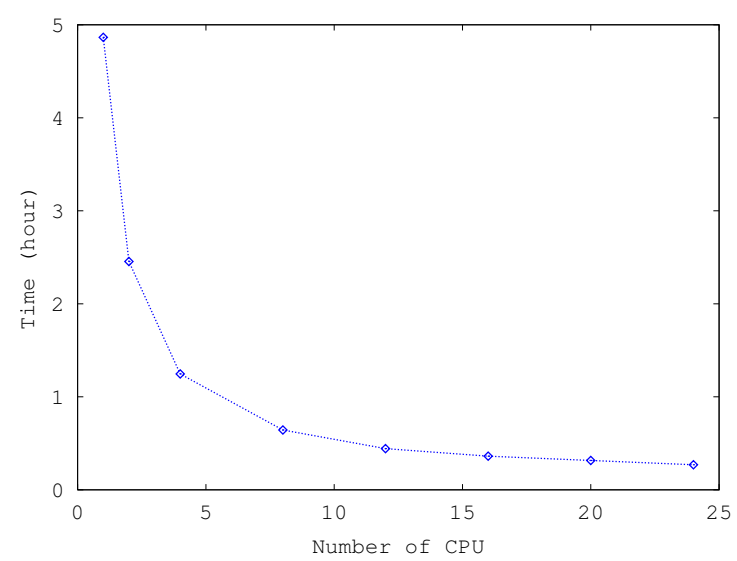

(a)

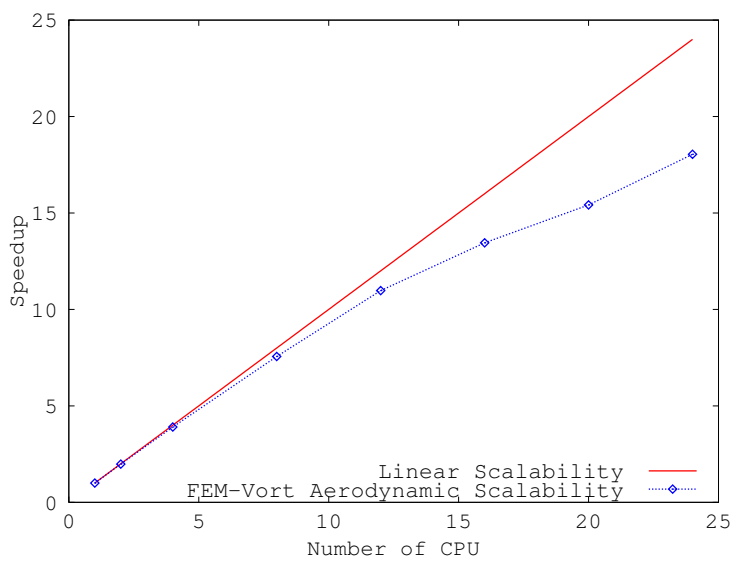

(b)

Figure 3.9: Scalability of parallel implementation: 17 meter DOE-Sandia VAWT, 23 blade elements, 10 revolutions, 40 steps per revolution (a) Computer run time (b) Speedup

\subsection{Conclusions}

An already developed free vortex model which is based on vortex filaments is adopted in this chapter with two modifications: the effect of variable Reynolds number is incorporated; moreover, the most time consuming routine of this model is parallelized using the shared 
memory parallel programming interface capabilities. The inclusion of the Reynolds number effect is demonstrated to change the tangential force predictions significantly. It is also highlighted that the parallel implementation scales well for this application. Furthermore, in this chapter, the suitable number of blade elements and time steps per revolutions are chosen through convergence analysis. These requirements will be used in the next chapter for the aeroelastic analysis of the 17-meter DOE-Sandia VAWT. 


\section{Chapter 4}

\section{Aeroelastic Analysis}

\subsection{Introduction}

This chapter details the methodology invoked in the aeroelastic analysis of VAWTs. The aeroelastic analysis is accomplished by coupling the structural dynamic equations, developed in Chapter 2, with the aerodynamic model, studied in Chapter 3.

The first section of this chapter describes the underlying concepts behind the coupling formulation. The time integration technique, the so-called average acceleration method, utilized for evaluating the structural displacement and velocity, is outlined. Then, the approach taken for updating the aerodynamic loads affected by the structural vibration is demonstrated.

The second part of this chapter is devoted to the results obtained from the full aeroelastic simulations of the 17-meter DOE-Sandia VAWT. The aerodynamic performance, including the predicted aerodynamic torque and the power curve, and the structural performance, particularly the vibratory stresses, are verified against the experimental data. 


\subsection{Aeroelastic Coupling}

Typically, in a fluid-structure interaction problem, the loose coupling between the two domains is carried out by following two steps: first the structural responses are evaluated due to the fluid loads, and then, the structural responses are used as a feedback to the fluid domain. Here, these two steps are described in more detail.

\subsubsection{Structural Deflection}

In Chapter 2, it was explained how the tangential $\left(C_{T}\right)$ and normal $\left(C_{N}\right)$ force coefficients are obtained for each blade element. Note that these coefficients are calculated at the Aerodynamic Centre (AC) located at the quarter chord point from the leading edge. It is worthwhile pointing out that the moment coefficient is assumed to be zero at AC. This assumption is particularly true for high Reynolds number regime. There exist small aerodynamic moments at relatively smaller Reynolds number, which is assumed to be negligible in this analysis.

In order to apply the tangential and normal aerodynamic forces on the structure, these forces have to be moved to the Elastic Axis (EA) of the structure. To perform this, a moment has to be applied at EA, as illustrated in Fig.4.].a. The non-dimensional magnitude of this moment is $C_{M}=C_{N} \frac{d^{0}}{b}$, where $\frac{d^{0}}{b}$ is the non-dimensional distance between EA and AC with respect to the half chord length $b$. Referring to these coefficients, one can find the distributed forces $T$ and $N$, and the distributed moment $M$ over the the blade element as

$$
\begin{aligned}
T & =\frac{1}{2} \rho C C_{T} U_{R}^{2}, \\
N & =\frac{1}{2} \rho C C_{N} U_{R}^{2}, \\
M & =\frac{1}{2} \rho b C C_{M} U_{R}^{2},
\end{aligned}
$$


where $U_{R}$ is the blade velocity superimposed by the wind and vortex induced velocities. It is assumed that these forces are evenly distributed over the aerodynamic blade element.

An accurate prediction of the structural vibration demands sufficient number of FEM elements. This number of FEM elements are typically greater than the ones needed for the aerodynamic model. Therefore, each aerodynamic blade element might constitute of one to several FEM elements. The aerodynamic load distribution calculated for any aerodynamic blade element in Eq.4.], could be attributed to the FEM elements belong to it. Hence, depending on the length of the FEM element, one could easily assign half of the distribution to each node of the FEM element, representing the FEM nodal forces. Referring to the rotating frame of reference shown in Fig.4.1.b, $N$ and $T$ are aligned along $\mathbf{e}_{\mathbf{1}}$ and $\mathbf{e}_{\mathbf{2}}$, and $M$ is about $\mathbf{e}_{3}$. However, the signs have to be decided based on the directions of the frame of reference axes.

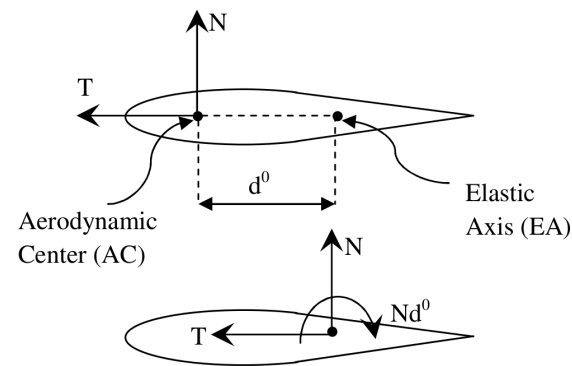

(a)

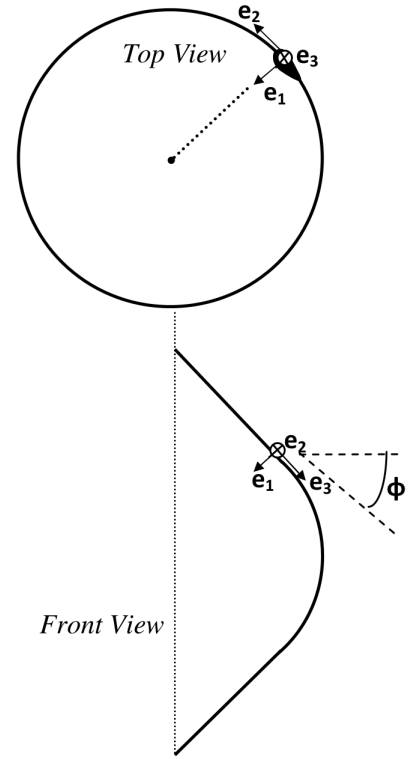

(b)

Figure 4.1: (a) Aerodynamic forces on the blade section (b) Rotating frame of reference 
Once the aerodynamic forces are properly incorporated in the right hand side of the following structural dynamic equation,

$$
M \ddot{\widetilde{y}}_{D}+C \dot{\widetilde{y}}_{D}+K \widetilde{y}_{D}=F
$$

this equation is solved in time. The time integration technique used in this analysis is the so-called average acceleration method. This technique is an implicit scheme and unconditionally stable. The steps are as follows:

\section{Algorithm: Average Acceleration Method}

inputs: initial displacement $\widetilde{y}_{D 0}$, initial velocity $\dot{\widetilde{y}}_{D 0}$

1 compute: initial acceleration $\ddot{\widetilde{y}}_{D 0}=M^{-1}\left(F_{0}-C \dot{\tilde{y}}_{D 0}-K \widetilde{y}_{D 0}\right)$

2 for $i=1,2, \ldots, n_{a}$ : total number of aerodynamic updates

3 for $j=1,2, \ldots, n_{s}$ : number of structural calculations between two aerodynamic updates

$4 \quad k=(i-1) n_{s}+j$

$5 \quad$ compute: $\widetilde{y}_{D k+1}=\left[\frac{4}{h^{2}} M+\frac{2}{h} C+K\right]^{-1}\left(F_{i+1}+M\left(\frac{4}{h^{2}} \widetilde{y}_{D k}+\frac{4}{h} \dot{\tilde{y}}_{D k}+\ddot{\widetilde{y}}_{D k}\right)+C\left(\frac{2}{h} \widetilde{y}_{D k}+\dot{\widetilde{y}}_{D k}\right)\right)$

6 compute: $\dot{\widetilde{y}}_{D k+1}=-\dot{\widetilde{y}}_{D k}+\frac{2}{h}\left(\widetilde{y}_{D k+1}-\widetilde{y}_{D k}\right)$

7 compute: $\ddot{\widetilde{y}}_{D k+1}=-\ddot{\widetilde{y}}_{D k}+\frac{4}{h^{2}}\left(\widetilde{y}_{D k+1}-\widetilde{y}_{D k}-h \dot{\widetilde{y}}_{D k}\right)$

8 end for

9 end for

In this algorithm, $h$ is the time step for the structural calculation. Since the computer run time for the structural calculation is significantly less than the aerodynamic calculation, the average acceleration method is implemented such that several structural calculations can 
be performed between the two aerodynamic updates. Of course, for each set of these calculations, the aerodynamic forces are assumed to be unchanged, having the most updated value.

\subsubsection{Aerodynamic Updates}

Following the structural vibration, the aerodynamic forces have to be adapted such that they account for this vibration. With regards to the aerodynamic formulation presented in Chapter 2, two adjustments seem to be necessary to impose on the fluid domain:

- In Chapter 3, the Aerodynamic Centre (AC) of each section of the blade was assumed to travel the perimeter of a full circle. This is a true assumption when the structure is considered to be rigid. However, flexibility of the structure violates this assumption in a sense that the deformation of the structure causes $\mathrm{AC}$ to deviate from a circular path.

- Vibration induced velocities have to be also superimposed on the blade, free stream and the vortex induced velocities.

For a full aeroelastic simulation these effects have to be taken into account. A more detailed discussion is presented next.

\section{Structural Displacement}

In Chapter 3, the blades were assumed to be rigid. Hence, the location of the aerodynamic centre (AC) of any blade section was dictated by the equation of a circle, as follows 


$$
\begin{aligned}
\theta_{B} & =\frac{2 \pi}{N B}(B N-1)+\theta, \\
X & =-r \sin \theta_{B}, \\
Z & =-r \cos \theta_{B},
\end{aligned}
$$

where $\langle X, Y, Z\rangle$ is the global coordinates of $\mathrm{AC}$ of any section of the blades, and $\theta_{B}$ is the blade azimuth angle.

Assuming a more realistic case, where the blades are flexible, allows AC of the blade section to vibrate about the rigid body position. Although this deflection is relatively small compared to the rigid body motion, it has to be considered in an aeroelastic analysis. Note that the structural displacement $\widetilde{y}_{D}$ obtained as the output of the average acceleration method at each time step, are the displacements of the Elastic Axis (EA) in the rotating frame of reference depicted in Fig.2. D.a. Hence, one has to relate the movements of AC to the EA displacements. Moreover, because the motion of the blades and the vortices in the aerodynamic model are tracked in a stationary coordinate system shown in Fig. B.].b, the displacements of AC has to be transformed into this coordinate system. The details of this process is discussed in the second section of Appendix A. Based on that, Eq.4.3 has to be updated for the structural displacement as follows

$$
\begin{aligned}
\theta_{B} & =\frac{2 \pi}{N B}(B N-1)+\theta, \\
X & =-r \sin \theta_{B}+\Delta X, \\
Z & =-r \cos \theta_{B}+\Delta Z,
\end{aligned}
$$

and $\Delta Y$ has to be added to the values obtained from the troposkien geometry. $\Delta X, \Delta Y$, 
$\Delta Z$ are defined as

$$
\begin{aligned}
& \Delta X=m \sin \theta_{B}-v \cos \theta_{B}, \\
& \Delta Y=n, \\
& \Delta Z=m \cos \theta_{B}-v \sin \theta_{B},
\end{aligned}
$$

where

$$
\begin{gathered}
m=\left(d^{0} \chi_{1}-w\right) \cos \phi+\left(u+d^{0} \alpha\right) \sin \phi, \\
n=\left(u-d^{0} \alpha\right) \cos \phi+\left(d^{0} \chi_{1}-w\right) \sin \phi,
\end{gathered}
$$

and $y_{D}=\left\{u \chi_{1} v \chi_{2} w \alpha\right\}^{T}$ is the displacement of the elastic axis, and $\phi$ is shown in Fig.t.d.b.

Now that the location of the $\mathrm{AC}$ points are updated due to the structural displacement, let us revisit the local coordinate system previously defined for rigid blades in Section B.2.1, i.e.,

$$
\begin{aligned}
& \mathbf{s}=\frac{\left(X_{i+1}-X_{i}\right) \mathbf{i}+\left(Y_{i+1}-Y_{i}\right) \mathbf{j}+\left(Z_{i+1}-Z_{i}\right) \mathbf{k}}{\sqrt{\left(X_{i+1}-X_{i}\right)^{2}+\left(Y_{i+1}-Y_{i}\right)^{2}+\left(Z_{i+1}-Z_{i}\right)^{2}}}, \\
& \mathbf{c}=\cos \theta_{B} \mathbf{i}-\sin \theta_{B} \mathbf{k}, \\
& \mathbf{n}=\mathbf{s} \times \mathbf{c}
\end{aligned}
$$

where $\left\langle X_{i}, Y_{i}, Z_{i}\right\rangle$ is $i$ the $\mathrm{AC}$ along the blade. Note that updating the coordinates of the aerodynamic centre due to structural deflection, as described earlier, automatically revises the vector $\mathbf{s}$. Besides $\mathbf{s}$, the vector $\mathbf{c}$, which represents the chordwise direction of the blade section, has to also account for the structural deflection. This is explained in the third 
section of Appendix A in detail. Based on the results, Eq.4.6 has to be updated as given by

$$
\begin{aligned}
& \mathbf{s}=\frac{\left(X_{i+1}-X_{i}\right) \mathbf{i}+\left(Y_{i+1}-Y_{i}\right) \mathbf{j}+\left(Z_{i+1}-Z_{i}\right) \mathbf{k}}{\sqrt{\left(X_{i+1}-X_{i}\right)^{2}+\left(Y_{i+1}-Y_{i}\right)^{2}+\left(Z_{i+1}-Z_{i}\right)^{2}}} \\
& \mathbf{c}=\cos \theta_{B} \mathbf{i}-\sin \theta_{B} \mathbf{k}+\Delta \mathbf{c}, \\
& \mathbf{n}=\mathbf{s} \times \mathbf{c}
\end{aligned}
$$

where $\Delta \mathbf{c}$ is defined as

$$
\Delta \mathbf{c}=\left\{\begin{array}{c}
-\left(\alpha \sin \phi+\chi_{1} \cos \phi\right) \sin \theta_{B} \\
\alpha \cos \phi-\chi_{1} \sin \phi \\
-\left(\alpha \sin \phi+\chi_{1} \cos \phi\right) \cos \theta_{B}
\end{array}\right\}
$$

\section{Structural Velocities}

In Chapter 3, the following equation was used to calculate the velocities of AC of any section of the rigid blade

$$
\mathbf{U}_{\mathbf{R}}=\left(U+U_{\infty}+U_{t} \cos \theta_{B}\right) \mathbf{i}+V \mathbf{j}+\left(W-U_{t} \sin \theta_{B}\right) \mathbf{k}
$$

where $U_{t}$ is the blade rotational velocity, $U_{\infty}$ is the free stream velocity and $\langle U, V, W\rangle$ is the induced velocity caused by the vortices. However, for the aeroelastic analysis the vibration induced velocity has to be added to this equation. In the previous sections, it was shown how solving the structural dynamic equation could result in evaluating the structural velocity $\dot{\widetilde{y}}_{D}$ at the elastic axis. In order to exploit this velocity in the aerodynamic model, they have to be transformed to the aerodynamic centre of the blade section, with respect to 
the $\langle\mathbf{i}, \mathbf{j}, \mathbf{k}\rangle$ coordinate. The required equations are derived in the last section of Appendix A. Referring to these equations, Eq. 4.9 has to be updated as follows

$$
\mathbf{U}_{\mathbf{R}}=\left(U+U_{\infty}+U_{t} \cos \theta_{B}+\dot{X}\right) \mathbf{i}+(V+\dot{Y}) \mathbf{j}+\left(W-U_{t} \sin \theta_{B}+\dot{Z}\right) \mathbf{k},
$$

where

$$
\begin{aligned}
& \dot{X}=(\dot{m}-\Omega v) \sin \theta_{B}-(\dot{v}-\Omega(m+r)) \cos \theta_{B}, \\
& \dot{Y}=\dot{n}, \\
& \dot{Z}=(\dot{m}-\Omega m) \cos \theta_{B}-(\dot{v}-\Omega(v-r)) \sin \theta_{B},
\end{aligned}
$$

$\dot{m}$ and $\dot{n}$ are defined as

$$
\begin{aligned}
\dot{m} & =\left(d^{0} \dot{\chi}_{1}-\dot{w}\right) \cos \phi+\left(\dot{u}+d^{0} \dot{\alpha}\right) \sin \phi, \\
\dot{n} & =\left(\dot{u}-d^{0} \dot{\alpha}\right) \cos \phi+\left(d^{0} \dot{\chi}_{1}-\dot{w}\right) \sin \phi,
\end{aligned}
$$

and $\dot{y}_{D}=\left\{\dot{u} \dot{\chi}_{1} \dot{v} \dot{\chi}_{2} \dot{w} \dot{\alpha}\right\}^{T}$ is the velocity of the elastic axis of the blade section.

\subsection{Results and Discussions}

Here, the aeroelastic results of FEM-Vort for the analysis of 17-meter DOE-Sandia VAWT is presented. Aerodynamic performance of this VAWT is discussed in terms of the generated torque and power curve. Furthermore, the structural performance is demonstrated through the structural parameters such as displacement, internal forces, strain and stress. 


\subsubsection{Aerodynamic Performance}

\section{Aerodynamic Torque}

Fig.42 illustrates the comparison of FEM-Vort torque predictions with the experimental data. The experimental data is obtained from the measurements performed by McNerney [27] on the 17-meter DOE-Sandia VAWT. Shown in this figure, at the relatively high Tip Speed Ratio $(T S R)$, i.e., high ratio of the blade velocity at the equator to the wind velocity, in this case 4.36, the agreement between FEM-Vort and the experimental data is excellent. However, for the lower values of $T S R$, FEM-Vort demonstrates poor predictions. This is implied at $T S R=2.8$ and $T S R=2.18$. Berg [R8] reports that this mainly hinges on the dynamic stall phenomenon: At relatively high tip speed ratio, due to the higher magnitude of the blade velocity, the relative velocity creates a smaller angle with the blade velocity. Therefore, the angle of attack becomes lower. For a typical $T S R$ of 4 or greater, the angle of attack of the equator and the sections close to it, vary in a range which is less than the static stall angle of attack. Note that the sections close to the equator are mostly responsible for generating the torque due to the higher radius (arm). Hence, dynamic stall does not play a crucial role. However, at the lower tip speed ratios, such as 2.8 and 2.18, the maximum angle of attack of the equator exceeds the static stall angle. Therefore, the blade periodically transitions between the stalled and unstalled conditions. This fact unfolds the necessity for including the dynamic stall model in FEM-Vort. Berg [28] incorporates a modified Boeing-Vertol dynamic stall model into VDART3 and shows that the results for the torque predictions improve significantly. 


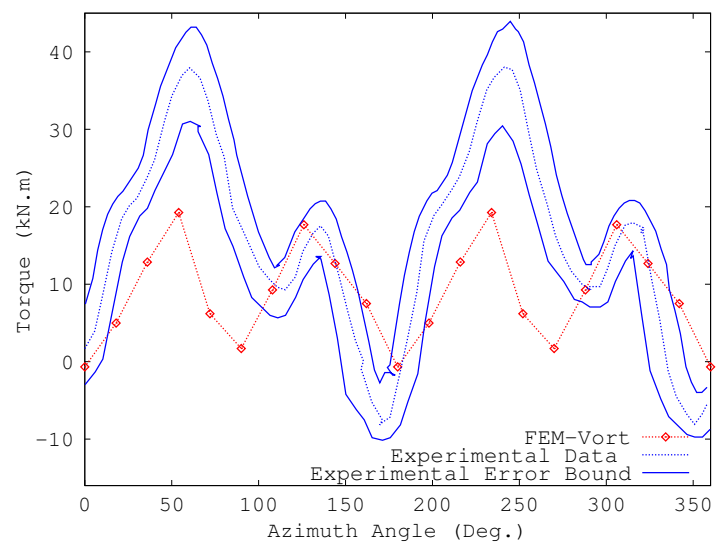

(a)

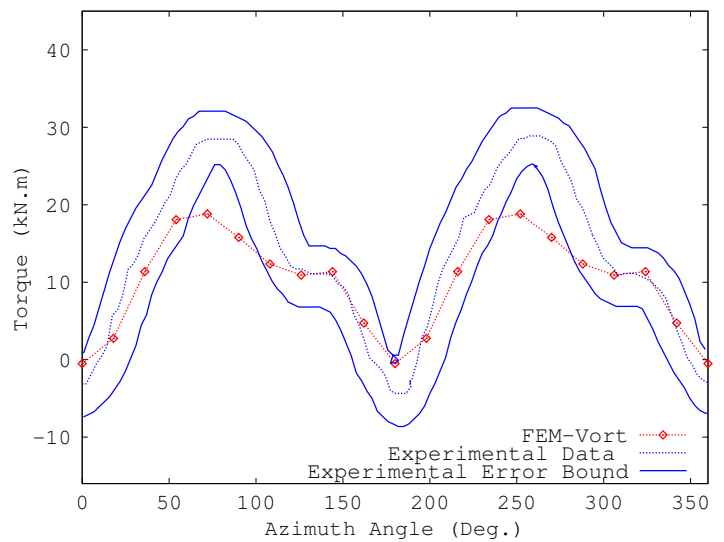

(b)

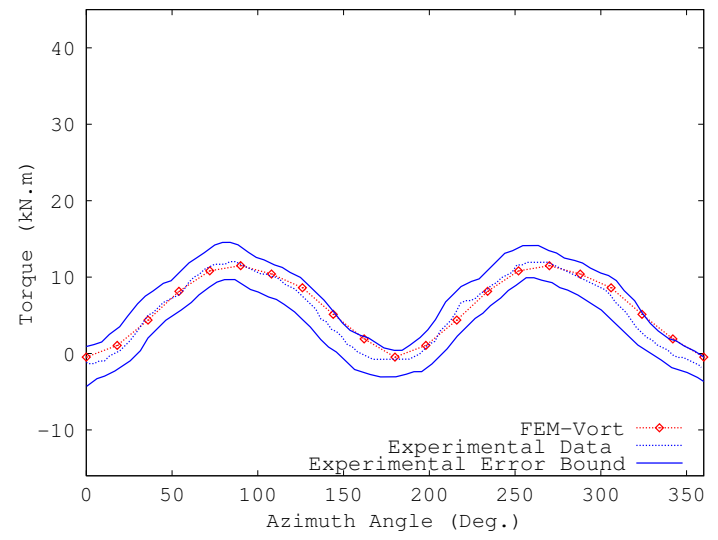

(c)

Figure 4.2: Comparison of the generated torque: 17-meter DOE-Sandia VAWT, operating angular velocity $\Omega=50.6 \mathrm{rpm}$ (a) Tip speed ratio $=2.18$

(b) Tip speed ratio $=2.8$ (c) Tip speed ratio $=4.36$ 


\section{Power Curve}

Fig.4.3 presents the power curve predicted by FEM-Vort for the 17-meter DOE-Sandia VAWT at the operating angular velocity of $50.6 \mathrm{rpm}$. The experimental data, obtained from the reference [ [0]], is also plotted. Although the trend of the experimental data is predicted reasonably, there exists an offset between the values. At low tip speed ratio, this offset is mainly due to the lack of dynamic stall model in the calculations as discussed earlier. However, the discrepancies exist at high tip speed ratios have to be investigated further.

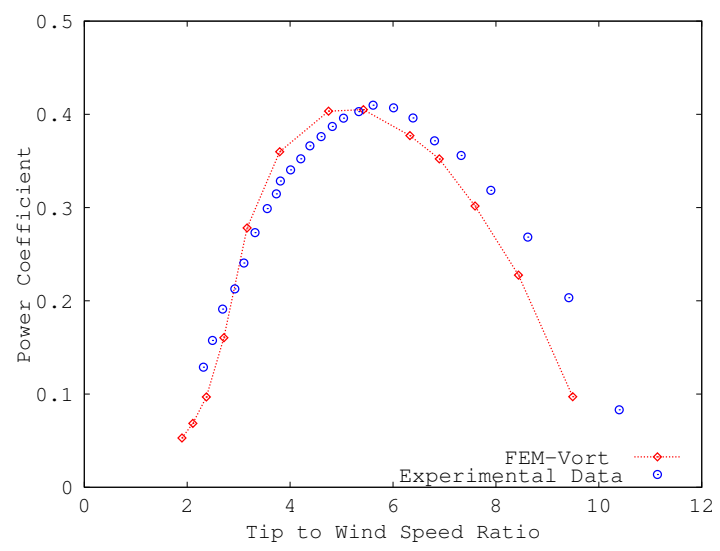

Figure 4.3: The power curve of the DOE-Sandia 17 meter VAWT: operating angular velocity $\Omega=50.6 \mathrm{rpm}$

\section{Wake Structure}

Fig.4.4 shows the wake structure of the 17-meter DOE-Sandia VAWT when the azimuth angle $\theta$ becomes $1890^{\circ}$ or $90^{\circ}$ after completing five revolutions. At this position, the chordwise axis of the blades are perpendicular to the direction of the wind. The upwind blade generates maximum torque while the generated torque by the downwind blade is damped out due to the strong interaction with the vortices. In Fig.4.4, the colours assigned to the vortices are chosen based on their evolution in time. The vortices with the blue colour left 
the blades initially, while, the red colour vortices have just initiated their movements. The points shown here as the vortices belong to the start and end nodes of the vortex filaments when convecting downstream of the wind turbine. This figure implies that the movement of the vortices at the core is delayed by the strong interaction with the other vortices.

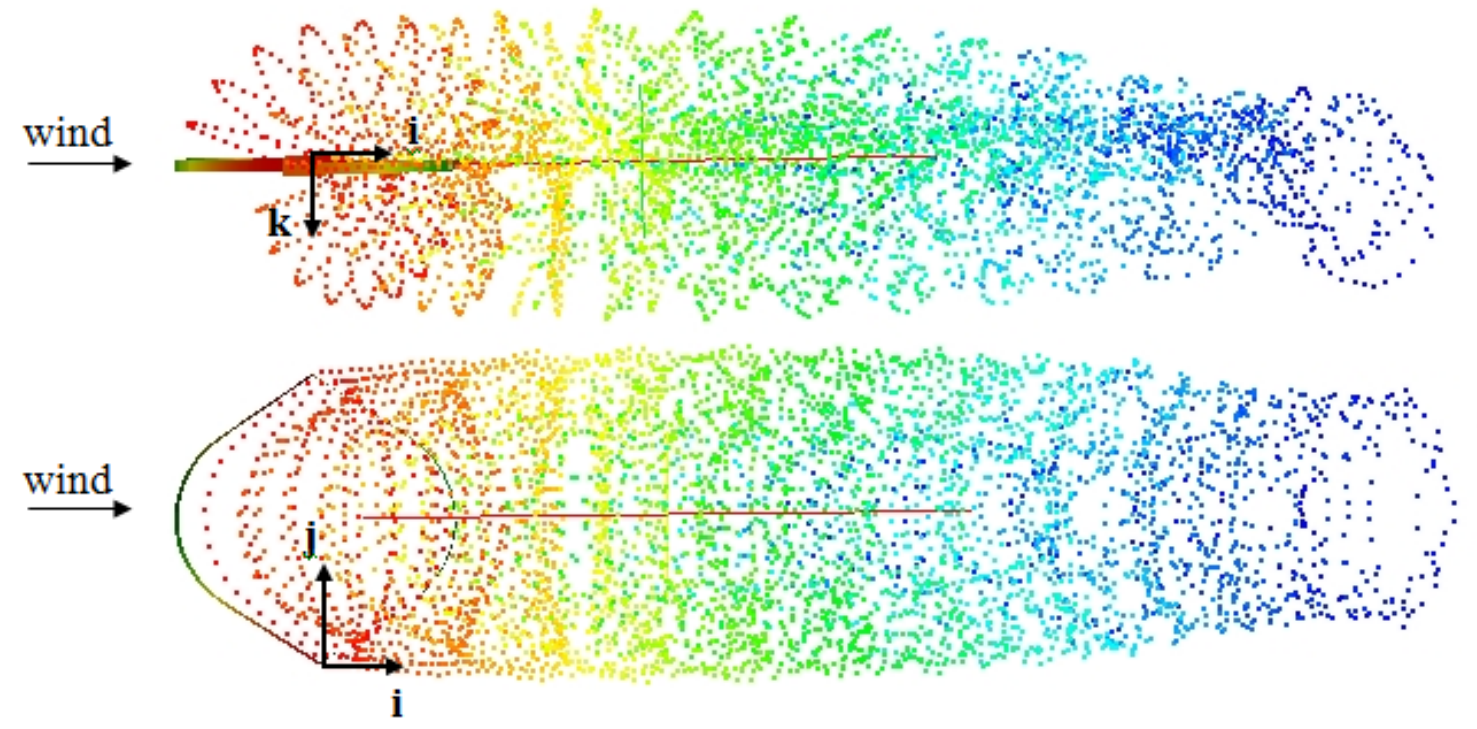

Figure 4.4: Wake structure: 17-meter DOE-Sandia VAWT, operating angular velocity $\Omega=50.6 \mathrm{rpm}$, tip speed ratio $=4.36$, azimuth angle

$$
(\theta)=1890^{\circ}
$$

\subsubsection{Structural Performance}

\section{Structural Displacement}

The Elastic Axis (EA) displacements of the 17-meter DOE-Sandia VAWT at the equator are shown in Fig.4.5. Due to the symmetry of the structure with respect to the equator and the lack of gravitational forces in the model, the plunge $\left(\chi_{1}\right)$ and chordwise $\left(\chi_{2}\right)$ rotational displacements as well as the spanwise translational displacement $(w)$ are zero. Fig.t.5 shows that the maximum steady-state amplitudes of the plunge $(u)$, chordwise $(v)$ and 
pitch $(\alpha)$ displacements are $25 \mathrm{~mm}, 7.5 \mathrm{~mm}$ and $0.08^{\circ}$ respectively. The relatively small magnitude of these degrees of freedom proves that the effect of the structural displacements in changing the aerodynamic forces is minimal.

Fig.E.6 presents the frequency content of the aforementioned displacements. The DC term of the frequency content of $u$ exhibits a value of about $25 \mathrm{~mm}$. This is the mean value of the plunge displacement evident in Fig.4.5. a as well. The main cause of this relatively large mean value is the steady-state centrifugal force manifest itself in vector $f_{s s}$ in Eq.2.19. The vibratory part of this displacement comes from the aerodynamic forces, representing the non-zero frequency components. The zero-frequency term for $v$ is $2.5 \mathrm{~mm}$ approximately. Since the centre of mass offset is assumed to be zero in this analysis, i.e., $x_{\alpha}=0$, the chordwise component of the centrifugal force $f_{s s}$ is zero as well. Hence the response $v$ is purely aerodynamic dependent. The mean offset of $v$ conforms with the fact that the tangential force and consequently the generated torque depicted in Fig.4.2 oscillates about a non-zero mean. Moreover, it is noticeable that the other dominant frequency components of $v$ response are the first four harmonics, i.e., $\Omega, 2 \Omega, 3 \Omega$ and $4 \Omega$, where $\Omega$ is the angular velocity of wind turbine, in this case $5.3 \mathrm{rad} / \mathrm{s}$. Similarly, these harmonics are also present in the frequency content of the pitch displacement $\alpha$, shown in Fig.4.6.b. Note that the mean value of $\alpha$ is a response to only aerodynamic forces because the centrifugal spanwise moment $f_{s s}^{6}=-b x_{\alpha} r \sin \phi$ is zero.

It is worthwhile pointing out that herein, the initial displacement $\widetilde{y}_{D 0}$ and velocity $\dot{\widetilde{y}}_{D 0}$, required in the calculation of the Average Acceleration Method, are assumed to be zero. The influence of such an assumption is evident in the transient response of the system shown in Fig.4.7. According to this figure, the transient response disappears after almost two revolutions of the wind turbine. This is due to the structural damping mechanism introduced to the system. The structural damping used in this analysis is a stiffness proportional 
damping that introduces $5 \%$ damping to the 10th natural frequency of the system. This means that the first to the 9th natural frequencies experience less than $5 \%$; conversely, the rest experience more than $5 \%$ damping. This type of damping guarantees that the solution is not smeared by any spurious high frequency component.

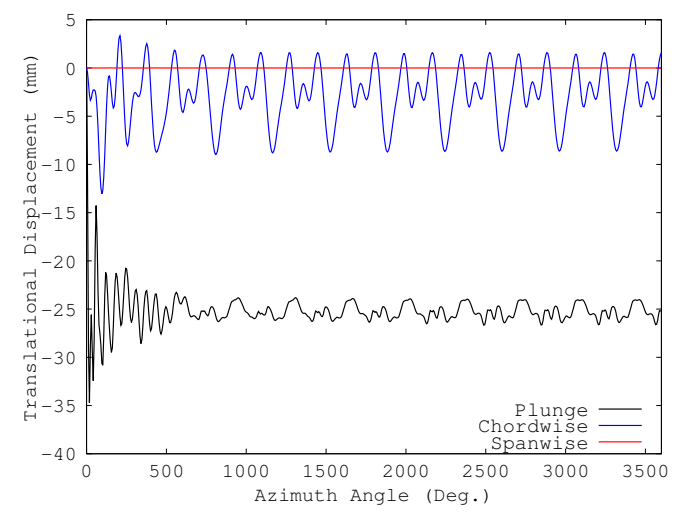

(a)

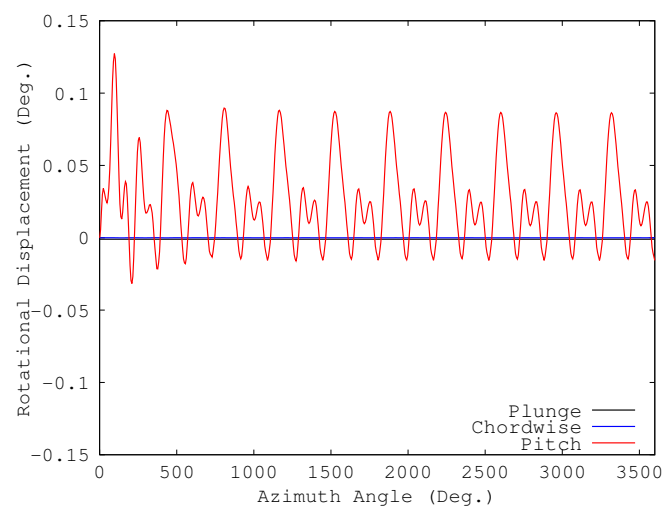

(b)

Figure 4.5: Elastic axis displacement at the equator of the 17-meter DOE-Sandia VAWT: operating angular velocity $\Omega=50.6 \mathrm{rpm}$, tip speed ratio $=4.36$ (a) Translational displacement (b) Rotational displacement

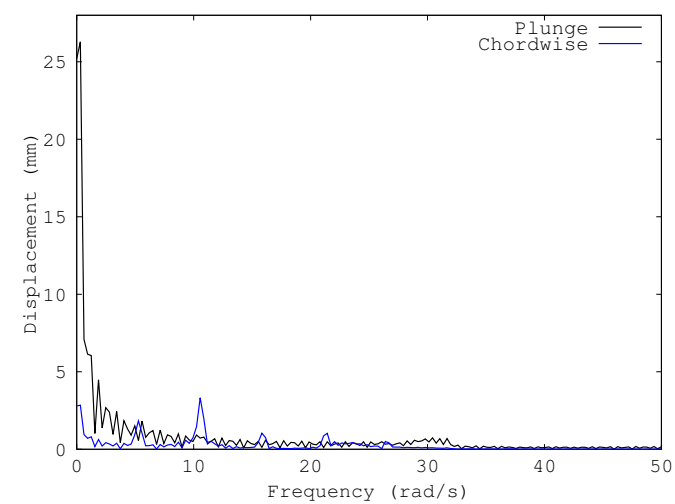

(a)

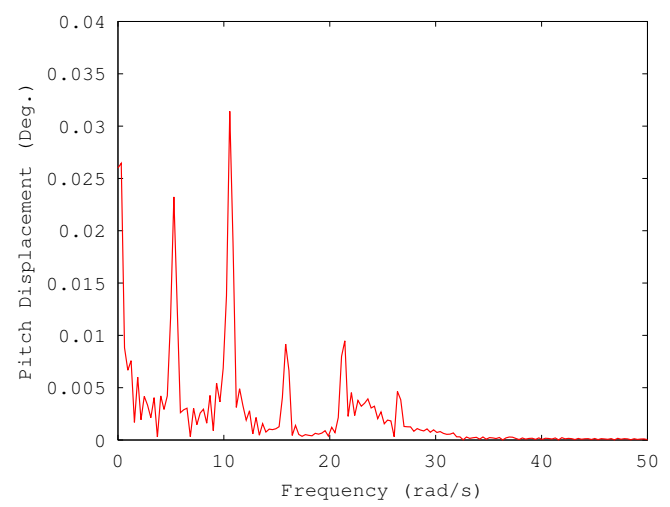

(b)

Figure 4.6: Frequency content of the elastic axis displacement at the equator of the 17-meter DOE-Sandia VAWT: operating angular velocity $\Omega=50.6 \mathrm{rpm}$, tip speed ratio $=4.36$ (a) Translational displacement (b)

Pitch displacement 


\section{Internal Forces}

One of the advantages of the mixed finite element formulation presented in Chapter 2 relies on the fact that following the calculation of the generalized displacement vector $y_{D}=$ $\left\{u \chi_{1} v \chi_{2} w \alpha\right\}^{T}$ from Eq.2.23, the generalized force vector $y_{F}=\left\{Q_{1} M_{1} Q_{2} M_{2} \tau M_{3}\right\}^{T}$ could easily be evaluated by using the system of equations presented in Eq.2.16, i.e.,

$$
\widetilde{y}_{F}=-K_{F F}^{-1} K_{F D} \widetilde{y}_{D} .
$$

Fig.4.7 illustrates the internal forces at the root of the 17-meter DOE-Sandia VAWT, namely the moment about the plunge axis $M_{1}$, the moment about the chordwise axis $M_{2}$ and the axial force $\tau$. Two sets of results are plotted: the response to the complete set of external forces, i.e., aerodynamic and centrifugal forces, and the response to only the centrifugal forces. Referring to Fig.4.7.a, $M_{1}$ only consists of the response of the structure to the aerodynamic loading. On the contrary, the internal moment $M_{2}$ is combination of the mean value that arises from the centrifugal force and a vibratory part that comes from the aerodynamic load fluctuations. Likewise, $\tau$ is developed due to both aerodynamic and centrifugal forces.

\section{Strain \& Stress}

The cyclic nature of the aerodynamic loading underscores the necessity of the fatigue analysis for the VAWTs' blade. To carry out such an analysis, Veers [[] highlights that the time history of the axial stress is the most important factor to consider. Hence, the focus of this section is to predict the time history of the axial strain and stress. These quantities are found for the 17-meter DOE-Sandia VAWT. 


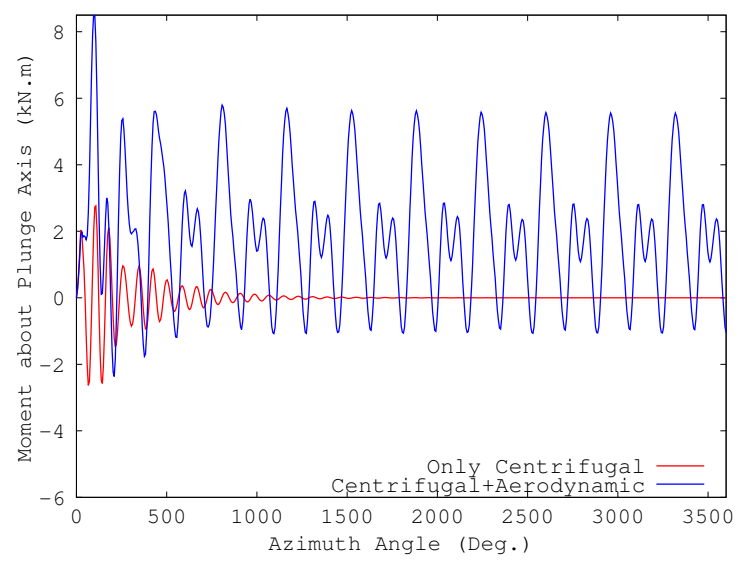

(a)

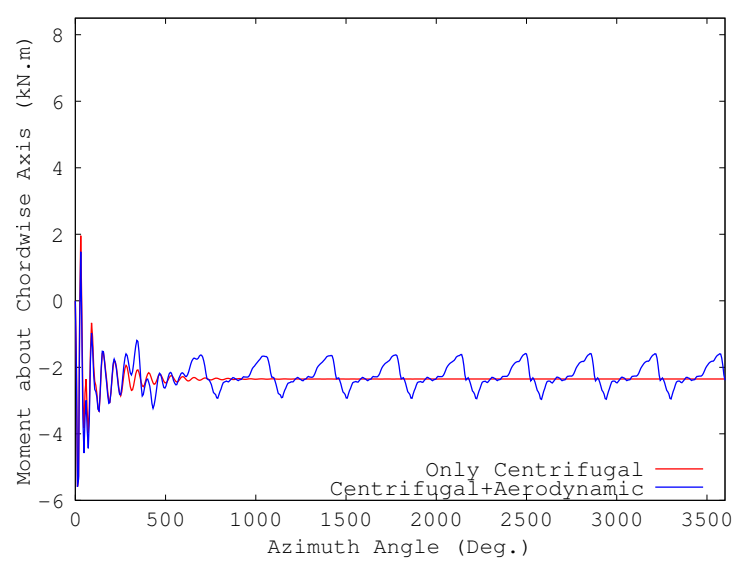

(b)

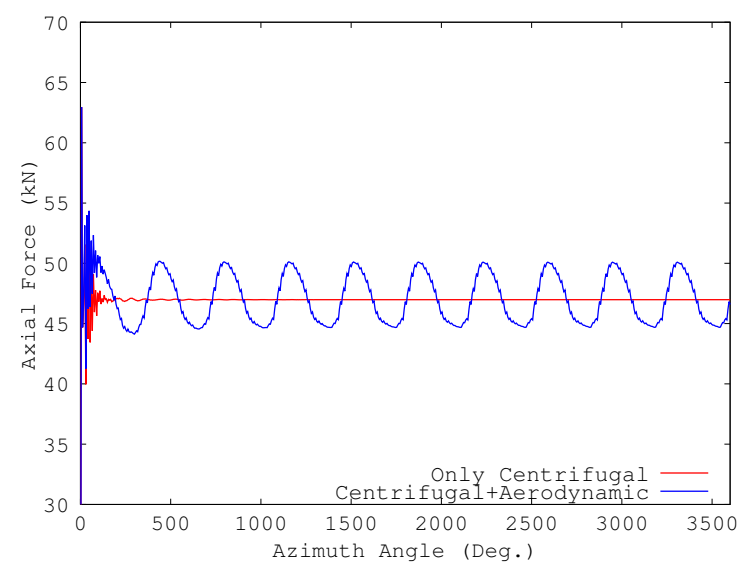

(c)

Figure 4.7: Elastic axis internal forces at the root of the 17-meter DOE-Sandia VAWT: operating angular velocity $\Omega=50.6 \mathrm{rpm}$, tip speed ratio $=4.36$ (a) Moment about plunge axis (b) Moment about chordwise axis (c) Axial force 
For calculating the axial strain and stress, the following equation is exploited, assuming that the neutral centre coincides with the elastic axis,

$$
\begin{aligned}
\epsilon_{s s} & =\frac{\tau}{E A}-\frac{M_{1} x}{E I_{y y}}+\frac{M_{2} y}{E I_{x x}}, \\
\sigma_{s s} & =E \epsilon_{s s},
\end{aligned}
$$

where $\left(y \mathbf{e}_{1}+x \mathbf{e}_{2}\right)$ is the location of any desired point on the blade section. The axial strain at the root of the blade is illustrated in Fig.4.8 for two locations: elastic axis and the trailing edge. The mean offset of the axial strain at the elastic axis is caused by the steady centrifugal force, and the vibratory part by the aerodynamic forces. The same trend happens for the axial strain at the trailing edge except that the response is amplified by the presence of the moment $M_{1}$. Fig.4.9 reveals the same behaviour for the stress.

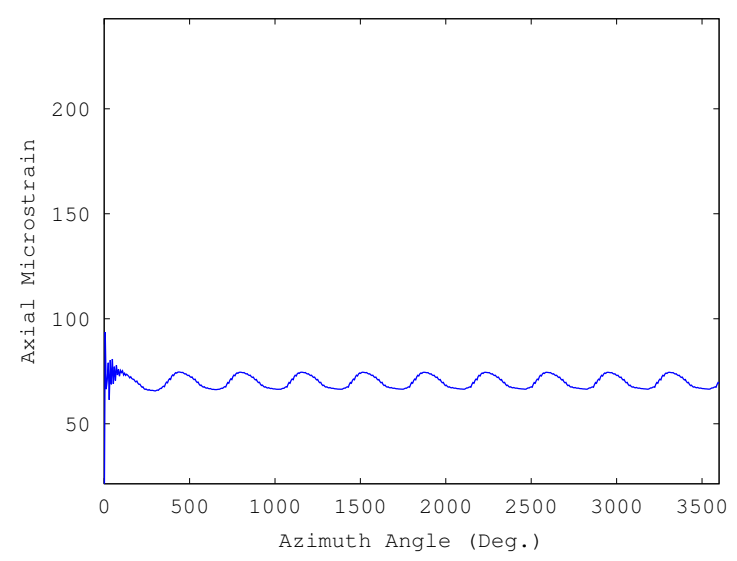

(a)

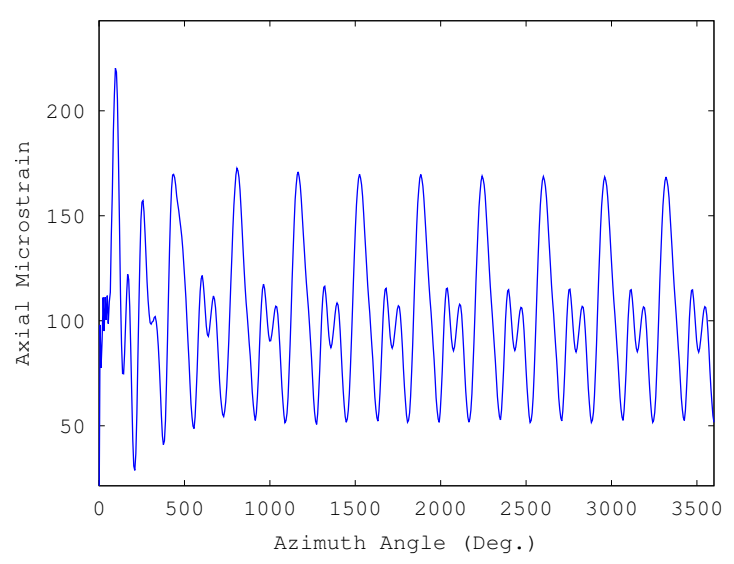

(b)

Figure 4.8: Axial strain at the root of the 17-meter DOE-Sandia VAWT:

operating angular velocity $\Omega=50.6 \mathrm{rpm}$, tip speed ratio $=4.36$ (a) Elastic axis (b) Trailing edge 


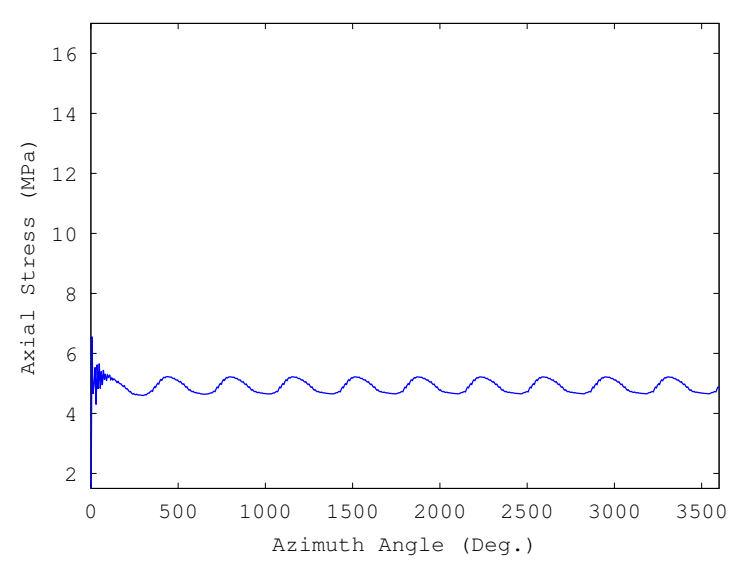

(a)

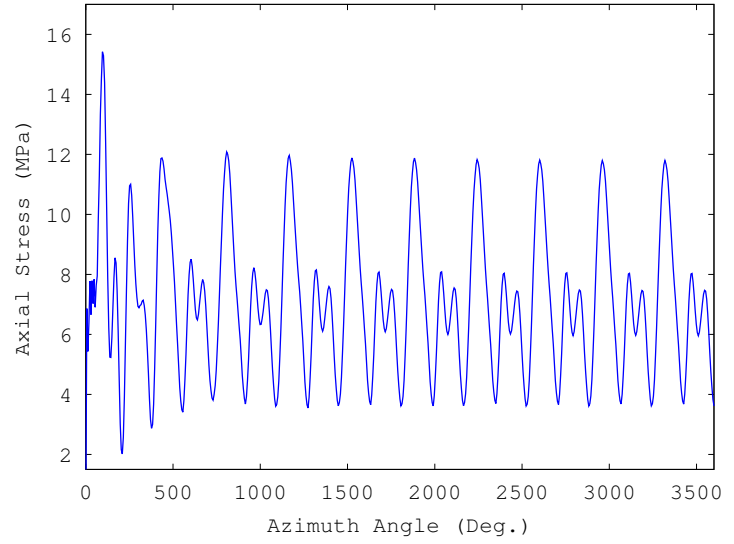

(b)

Figure 4.9: Axial stress at the root of the 17-meter DOE-Sandia VAWT: operating angular velocity $\Omega=50.6 \mathrm{rpm}$, tip speed ratio $=4.36$ (a) Elastic axis (b) Trailing edge

Fig.4.10 demonstrates the elastic axis axial stress along the blades of the 17-meter DOESandia VAWT. This snapshot is taken at the azimuth angle $(\theta)$ of $1890^{\circ}$ or $90^{\circ}$ after completing five revolutions. At this position, the elastic axis axial stress at the root of the upwind blade is at its peak. This is evident in Fig.4.9.a as well. Fig.4.10 reveals the fact that the roots of the blades experience higher stress comparing to the equator. This figure also demonstrates that the blades are at higher level of stress when they are in the upwind position. This is mainly due to the higher amount of aerodynamic forces at the upwind position when compared to the downwind position. 


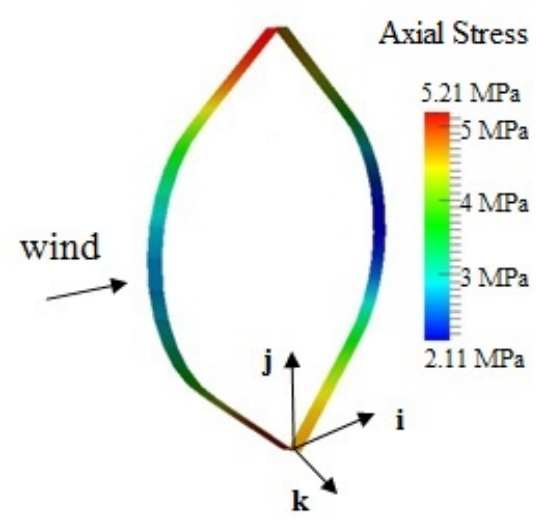

Figure 4.10: Elastic axis axial stress along the blades of the 17-meter DOE-Sandia VAWT: operating angular velocity $\Omega=50.6 \mathrm{rpm}$, tip speed ratio $=4.36$, azimuth angle $(\theta)=1890^{\circ}$

Finally, Fig.4. DOE-Sandia VAWT at different wind speed. In this figure, the results estimated by FEMVort are compared with the experimental data reported by Lobitz [ㅁ] ]. The vibratory stresses are taken to be half of the difference between maximum and minimum stresses. Implied in the definition of the vibratory stress, the effect of the steady state centrifugal forces are eliminated. Hence, the vibratory stress is merely linked to the aerodynamic oscillatory effects. Fig.t. س the experimental data when the tip speed ratio is relatively high, namely greater than 2.5. This range of tip speed ratios corresponds to the wind speeds less than $35 \mathrm{mph}$ for the operating angular velocity of $50.6 \mathrm{rpm}$. At low tip speed ratios, when the wind speed is higher than $35 \mathrm{mph}$, the numerical model developed here exhibits limited success in estimating the correct values of the vibratory stresses. This major limitation stems from inaccurate prediction of the aerodynamic loading at low tip speed ratios. As discussed earlier, this is mainly due to the absence of the dynamic stall model. 


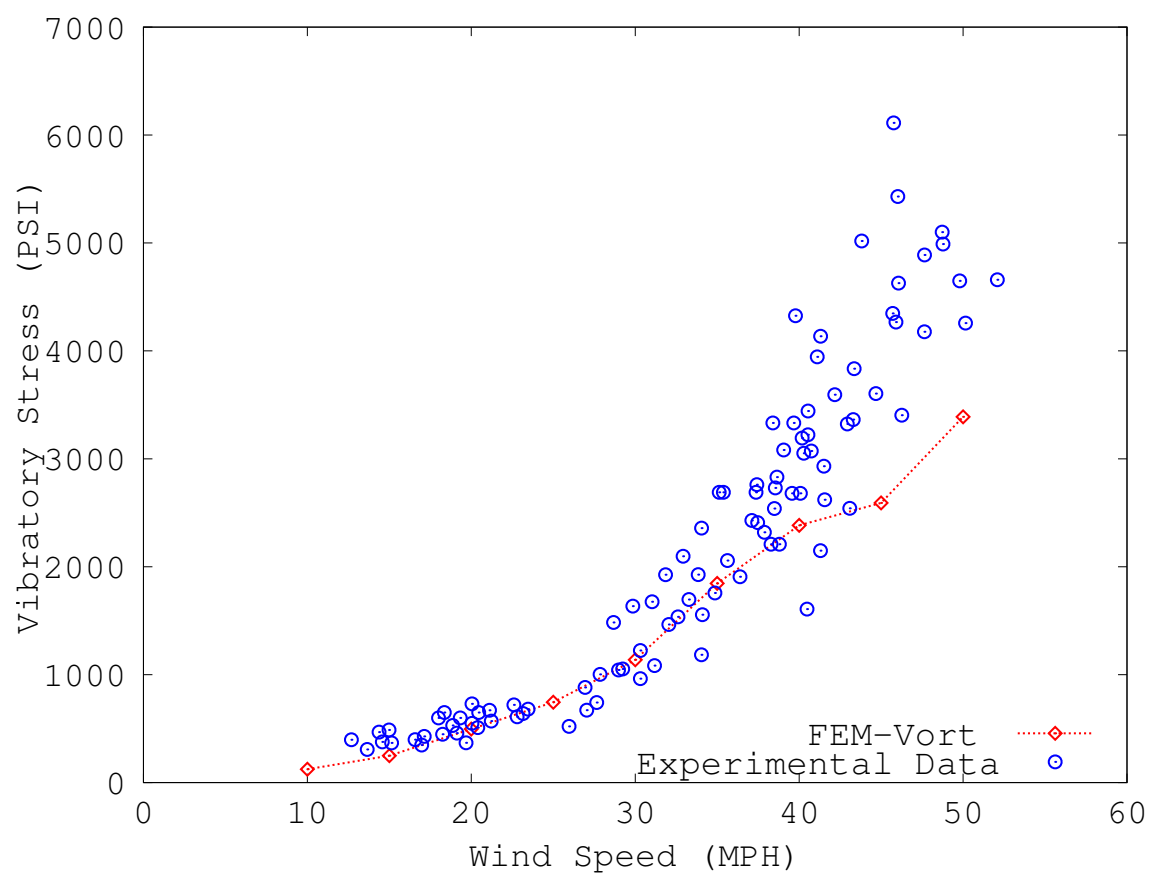

Figure 4.11: Trailing edge vibratory stress at the root of the DOE-Sandia 17-meter VAWT: operating angular velocity $\Omega=50.6 \mathrm{rpm}$

\subsection{Conclusions}

The specially devised structural dynamic equations for curved blades with troposkien shapes are coupled with the free vortex aerodynamic model. The accuracy of the aeroelastic model in predicting the aerodynamic performance is illustrated through torque and power estimations. It is shown that the model developed here is able to estimate the aerodynamic loads reasonably well except where the tip speed ratios are relatively low. This is mainly attributed to the lack of dynamic stall model in the current investigation. The same deficiency carries over when the numerically evaluated vibratory stresses are assessed. Except the region of the low tip speed ratios, this analysis provides a very good predictions of the stress in the blades of a VAWT. 


\section{Chapter 5}

\section{Conclusions and Recommendations}

\subsection{Summary}

A set of structural dynamic equations which describes the behaviour of a troposkien shape blade is treated in the finite element framework. The already developed structural dynamic equations were cast in the form of 12 first order ordinary differential equations in terms of generalized displacement and generalized force. The nature of the equations enforces a mixed finite element formulation derivation. This formulation is verified through modal analysis by inspecting the variation of the natural frequencies with respect to the angular velocity of the turbine. It is highlighted that the centrifugal stiffening effect prevents the system from dynamic instability. Furthermore, the effect of centre of mass offset and the Coriolis forces on the mode shapes of the structure are explored. These effects give rise to the coupling between certain degrees of freedom of the system. This system is assumed to vibrate in response to oscillating aerodynamic forces, hence it was required to incorporate an aerodynamic model.

A three dimensional free vortex model based on vortex filaments, already developed 
for VAWTs, is implemented. The effect of variable Reynolds number is augmented to the model. This feature alters the tangential force predictions substantially. Comparison between the predictions of this model and the experimental data reveals the capability of this model in estimating the aerodynamic forces especially for high tip speed ratios, where blade-vortex interaction becomes strong. However, the low tip speed ratios do not exhibit a good agreement mainly because dynamic stall is not considered in this aerodynamic model. Moreover, a simple parallel implementation of this model is shown to scale well with the increase of number of CPUs.

The two aforementioned models are coupled to form an aeroelastic model. This is carried out by calculating the structural responses to the aerodynamic and centrifugal forces in time using the average acceleration method. Then, the structural displacements and velocities are used to update the aerodynamic loading. Having developed this aeroelastic capability, the axial stresses along the blade are computed. The comparison of the vibratory stresses obtained from the analysis of the 17-meter DOE-Sandia VAWT with the experimental data shows an excellent agreement except where dynamic stall plays an important role.

\subsection{Conclusion}

The conclusion of the current thesis is summarized as following:

- The coupling between the linear structural dynamic equations developed by Nitzsche [10] and the vortex filament based aerodynamic model devised by Strickland [Q] provides a robust tool for investigating the aeroelastic behaviour of a VAWT in relatively high tip speed ratios.

- For providing reasonable estimation in low tip speed ratios, a suitable dynamic stall 
model has to be added to this model.

- This model lends itself well to shared memory based parallel implementation.

\subsection{Recommendations and Future Work}

Recommendations for improving the structural analysis:

- The current investigation neglects the effect of gravitational forces. As opposed to the centrifugal and aerodynamic loading, gravitational forces are unsymmetric with respect to the equator of the wind turbine. It is interesting to investigate the effect of this type of unsymmetric loading on the response of the structure, in combination with the aerodynamic and centrifugal forces.

- The structural dynamic equations used in this analysis are linear. Therefore, it will be very insightful to study the effect of geometric non-linearity in this problem. This becomes very important when the study of large deflections in more flexible blades is of interest.

Recommendations for improving the aerodynamic analysis:

- As emphasized throughout the thesis, the absence of dynamic stall model degraded the accuracy of the numerical predictions in low tip speed ratio regimes. Including a suitable dynamic stall model could enhance the results significantly. A wide range of dynamic stall models are available, ranging from CFD based models to pure empirical ones. For instance ONERA model is a semi-empirical dynamic stall model, which is very popular in helicopter applications. Perhaps applying the ONERA model could shed some light in understanding the dynamic stall mechanism in VAWTs. 
- In the current study, the wind velocity is assumed to be uniformly distributed along the height of the wind turbine. A more realistic case of study takes into account the atmospheric boundary layer effect. In the atmospheric boundary layer, the wind is generally gusty or turbulent, with the wind changing speed and direction rapidly. Considering this effect leads to more accurate predictions of the wind turbine performance.

- As a long term goal, it would be very compelling to replace the use of experimental data with a CFD based solution. The flow very close to the airfoil could be solved using CFD approaches and then the solution could be transferred to a vortex based approach in the wake of the airfoil. Although this coupled approach becomes computationally expensive, it would provide better resolution to the solution.

Future work for extending the application:

- The developed aeroelastic model showed promising results for the analysis of landbased VAWTs. This work could be extended for analyzing the behaviour of offshore VAWTs by implementing the effect of hydrodynamic forces in the base of the wind turbine.

- One of the disadvantages of VAWTs compared to HAWTs is the fatigue-related problems due to cyclic aerodynamic loading. The current model gives the opportunity for performing fatigue analysis by providing the time history of stress. Hence, a potential option for extending the current research is to use the predicted stresses for estimating the fatigue life of the blades.

- The current method of analysis is a relatively simple and robust tool for investigating the wind farm effects on the performance of individual wind turbines. 


\section{Appendix A}

\section{Appendix A: Aerodynamic Updates due to the Structural Vibration}

\section{A.1 Coordinate Systems and Transformation Matrices}

For the sake of a more convenient discussion in the following sections, four coordinate systems are defined here. The definitions of these coordinate systems, which are shown in Fig.A.], are as follows:

- $\mathbf{i}, \mathbf{j}, \mathrm{k}$ : the origin of this stationary coordinate system is placed at the bottom of the wind turbine.

$\mathrm{i}:$ is in the direction of the wind.

$\mathrm{j}$ : is in the direction of the vertical axis from the bottom to the top.

$\mathrm{k}: \mathrm{k}=\mathbf{i} \times \mathbf{j}$.

- $\mathbf{e}_{\mathbf{r}}, \mathbf{e}_{\theta}, \mathbf{e}_{\zeta}$ : the origin of this rotating coordinate system is placed at intersection of the local radius $r$, and the vertical axis of the turbine. This system rotates with the turbine's angular velocity $\Omega$. 
$\mathbf{e}_{\mathbf{r}}$ : is in the direction of the local radius $r$, outward the wind turbine.

$\mathbf{e}_{\theta}$ : is in the direction of the tangential velocity.

$\mathbf{e}_{\zeta}: \mathbf{e}_{\zeta}=\mathbf{e}_{\mathbf{r}} \times \mathbf{e}_{\theta}$, in the direction of $\mathbf{j}$.

- $\mathrm{e}_{1}, \mathrm{e}_{2}, \mathrm{e}_{3}$ : the origin of this rotating coordinate system is on the undisturbed elastic axis of the blade section.

$\mathbf{e}_{1}$ : is in the direction of the local curvature $\kappa^{(0)}$.

$\mathbf{e}_{2}$ : is in along the chord from trailing edge to the leading edge.

$\mathbf{e}_{3}: \mathbf{e}_{3}=\mathbf{e}_{1} \times \mathbf{e}_{2}$, tangent to the troposkien curve.

- $\mathrm{e}_{1}^{\prime}, \mathrm{e}_{2}^{\prime}, \mathrm{e}_{3}^{\prime}$ : the origin of this coordinate system is on the disturbed elastic axis of the blade section.

$\mathbf{e}_{1}^{\prime}$ : is in the direction of the local curvature $\kappa^{(0)}$ of the disturbed section.

$\mathbf{e}_{2}^{\prime}$ : is in along the chord from trailing edge to the leading edge of the disturbed section.

$\mathbf{e}_{3}^{\prime}: \mathbf{e}_{3}^{\prime}=\mathbf{e}_{1}^{\prime} \times \mathbf{e}_{2}^{\prime}$, tangent to the troposkien curve of the disturbed section.

The following transformation matrices are constructed for converting quantities between these coordinate systems:

$R_{1}$ :

$$
\left\{\begin{array}{l}
\mathbf{e}_{\mathbf{1}} \\
\mathbf{e}_{\mathbf{2}} \\
\mathbf{e}_{\mathbf{3}}
\end{array}\right\}=\left[\begin{array}{ccc}
1 & -\alpha & \chi_{2} \\
\alpha & 1 & -\chi_{1} \\
-\chi_{2} & \chi_{1} & 1
\end{array}\right]\left\{\begin{array}{l}
\mathbf{e}_{\mathbf{1}}^{\prime} \\
\mathbf{e}_{2}^{\prime} \\
\mathbf{e}_{\mathbf{3}}^{\prime}
\end{array}\right\},
$$


$R_{2}$ :

$$
\left\{\begin{array}{c}
\mathbf{e}_{\mathbf{r}} \\
\mathbf{e}_{\theta} \\
\mathbf{e}_{\zeta}
\end{array}\right\}=\left[\begin{array}{ccc}
-\sin \phi & 0 & \cos \phi \\
0 & 1 & 0 \\
-\cos \phi & 0 & -\sin \phi
\end{array}\right]\left\{\begin{array}{l}
\mathbf{e}_{\mathbf{1}} \\
\mathbf{e}_{\mathbf{2}} \\
\mathbf{e}_{\mathbf{3}}
\end{array}\right\}
$$

$R_{3}$ :

$$
\left\{\begin{array}{l}
\mathbf{i} \\
\mathbf{j} \\
\mathbf{k}
\end{array}\right\}=\left[\begin{array}{ccc}
-\sin \theta & -\cos \theta & 0 \\
0 & 0 & 1 \\
-\cos \theta & \sin \theta & 0
\end{array}\right]\left\{\begin{array}{l}
\mathbf{e}_{\mathbf{r}} \\
\mathbf{e}_{\theta} \\
\mathbf{e}_{\zeta}
\end{array}\right\}
$$

\section{A.2 Absolute Displacement of the Aerodynamic Centre due to the Structural Displacement}

This section is devoted to calculating the position of the aerodynamic centre after perturbation in the coordinate system $\langle\mathbf{i}, \mathbf{j}, \mathbf{k}\rangle$. Consider the following equation

$$
\langle X, Y, Z\rangle_{\text {disturbed }}=\langle X, Y, Z\rangle_{\text {undisturbed }}+\epsilon_{\mathbf{a c}}
$$

where $\epsilon_{\mathbf{a c}}$ is the disturbance of the aerodynamic centre. The undisturbed position of the aerodynamic centre can be obtained from the rigid body dynamics of the blade in the $\langle\mathbf{i}, \mathbf{j}, \mathbf{k}\rangle$ system, as given by

$$
\begin{aligned}
& X_{\text {undisturbed }}=-r \sin \theta, \\
& Z_{\text {undisturbed }}=-r \cos \theta,
\end{aligned}
$$


where $Y_{\text {undisturbed }}$ could be calculated from the troposkien geometry. Now, let us focus on calculating $\epsilon_{\mathbf{a c}}$ :

As shown in Fig. . I. b, the distance between the elastic axis and the aerodynamic centre before the vibration is $\mathbf{d}^{\mathbf{0}}=d^{0} \mathbf{e}_{\mathbf{2}}$, in the $\left\langle\mathbf{e}_{\mathbf{1}}, \mathbf{e}_{\mathbf{2}}, \mathbf{e}_{\mathbf{3}}\right\rangle$ coordinate system. Assume that the magnitude of $\mathrm{d}^{0}$ will be unchanged during the perturbation and it only undergoes pure rotation, denoted by $\mathbf{d}$ after perturbation. Using the transformation matrix $R_{1}$, one can find $\mathbf{d}$ in the $\left\langle\mathbf{e}_{\mathbf{1}}, \mathbf{e}_{\mathbf{2}}, \mathbf{e}_{\mathbf{3}}\right\rangle$ coordinate system, as given by

$$
\mathbf{d}=R_{1}^{T} \mathbf{d}_{\mathbf{0}}=d^{0}\left(\alpha \mathbf{e}_{\mathbf{1}}+\mathbf{e}_{\mathbf{2}}-\chi_{1} \mathbf{e}_{\mathbf{3}}\right)
$$

Referring to Fig.A.D.b, the disturbed position of the aerodynamic centre with respect to the undisturbed elastic axis, $\rho$, could be obtained as

$$
\rho=\epsilon_{\mathbf{e a}}+\mathbf{d}=\left(u+d^{0} \alpha\right) \mathbf{e}_{\mathbf{1}}+\left(v+d^{0}\right) \mathbf{e}_{\mathbf{2}}+\left(w-d^{0} \chi_{1}\right) \mathbf{e}_{\mathbf{3}}
$$

where $\epsilon_{\mathbf{e a}}$ is the disturbance of the elastic axis. On the other hand, one can write the disturbance of the aerodynamic centre $\left(\epsilon_{\mathbf{a c}}\right)$ as follows

$$
\epsilon_{\mathbf{a c}}=\rho-\mathbf{d}^{\mathbf{0}}=\left(u+d^{0} \alpha\right) \mathbf{e}_{1}+(v) \mathbf{e}_{2}+\left(w-d^{0} \chi_{1}\right) \mathbf{e}_{\mathbf{3}}
$$

Note that Eq. .8 shows the disturbance of the aerodynamic centre in the rotating coordinate system $\left\langle\mathbf{e}_{1}, \mathbf{e}_{2}, \mathbf{e}_{3}\right\rangle$. In order to update the locations of $\mathrm{AC}$ in the aerodynamic model, one has to represent this equation in the stationary coordinate system $\langle\mathbf{i}, \mathbf{j}, \mathbf{k}\rangle$. To perform this, the transformation matrices $R_{2}$ and $R_{3}$ have to be multiplied by $\epsilon_{\mathbf{a c}}$, i.e, $R_{3} R_{2} \epsilon_{\mathbf{a c}}$. This 
manipulation results in the following equation

$$
\epsilon_{\mathbf{a c}}=\left\{\begin{array}{c}
\Delta X \\
\Delta Y \\
\Delta Z
\end{array}\right\}=\left\{\begin{array}{c}
m \sin \theta-v \cos \theta \\
n \\
m \cos \theta-v \sin \theta
\end{array}\right\}
$$

where

$$
\begin{aligned}
& m=\left(d^{0} \chi_{1}-w\right) \cos \phi+\left(u+d^{0} \alpha\right) \sin \phi \\
& n=\left(u-d^{0} \alpha\right) \cos \phi+\left(d^{0} \chi_{1}-w\right) \sin \phi
\end{aligned}
$$

\section{A.3 Rotation of the Blade Section due to the Structural Displacement}

As described in the previous section, the chordwise axis of the blade undergoes only pure rotation due to the structural vibration, i.e., $d=d^{0}$. This is an important aspect to be considered when aerodynamic forces are being calculated. Referring to the previous section, the unit vector of this rotation vector could be calculated as

$$
\mathbf{c}=-\frac{\mathbf{d}}{d}=-\alpha \mathbf{e}_{1}-\mathbf{e}_{2}+\chi_{1} \mathbf{e}_{3}
$$

Note that Eq.A.T is expressed in terms of $\left\langle\mathbf{e}_{1}, \mathbf{e}_{2}, \mathbf{e}_{3}\right\rangle$, and it has to be transformed into the stationary coordinate system $\langle\mathbf{i}, \mathbf{j}, \mathbf{k}\rangle$. To conduct this, the transformation matrices $R_{2}$ 
and $R_{3}$ have to be used, the result is

$$
\mathbf{c}=-R_{3} R_{2} \frac{\mathbf{d}}{d}=\left\{\begin{array}{c}
\cos \theta-\left(\alpha \sin \phi+\chi_{1} \cos \phi\right) \sin \theta \\
\alpha \cos \phi-\chi_{1} \sin \phi \\
-\sin \theta-\left(\alpha \sin \phi+\chi_{1} \cos \phi\right) \cos \theta
\end{array}\right\}
$$

\section{A.4 Absolute Velocity of the Aerodynamic Centre due to the Structural Velocity}

This section aims at calculating the absolute velocity of the aerodynamic centre in the coordinate system $\langle\mathbf{i}, \mathbf{j}, \mathbf{k}\rangle$. The following well-known equation is used for this purpose

$$
\langle\dot{X}, \dot{Y}, \dot{Z}\rangle=\dot{\mathbf{r}}+\dot{\epsilon}_{\mathrm{ac}}+\Omega \times \epsilon_{\mathrm{ac}} .
$$

Note that the first term, $\dot{\mathbf{r}}$, can easily be expressed as $\dot{\mathbf{r}}=-r \Omega \mathbf{e}_{\theta}$. One can transform $\dot{\mathbf{r}}$ into the $\langle\mathbf{i}, \mathbf{j}, \mathbf{k}\rangle$ system by multiplying it with $R_{3}$, i.e.,

$$
\dot{\mathbf{r}}=r \Omega(\cos \theta \mathbf{i}-\sin \theta \mathbf{k}) .
$$

Furthermore, the second term of Eq.A.12, $\dot{\epsilon}_{\text {ac }}$, could be calculated by taking the derivative of Eq.A.9 in time,

$$
\dot{\epsilon}_{\mathbf{a c}}=\left\{\begin{array}{c}
\dot{m} \sin \theta-\dot{v} \cos \theta \\
\dot{n} \\
\dot{m} \cos \theta-\dot{v} \sin \theta
\end{array}\right\}
$$


where

$$
\begin{aligned}
& \dot{m}=\left(d^{0} \dot{\chi}_{1}-\dot{w}\right) \cos \phi+\left(\dot{u}+d^{0} \dot{\alpha}\right) \sin \phi \\
& \dot{n}=\left(\dot{u}-d^{0} \dot{\alpha}\right) \cos \phi+\left(d^{0} \dot{\chi}_{1}-\dot{w}\right) \sin \phi
\end{aligned}
$$

Finally, since $\Omega$ can be written as $\Omega=\Omega \mathbf{j}$, the last term of term of Eq. $\mathrm{A} \cdot 2, \Omega \times \epsilon_{\mathrm{ac}}$, is obtained as

$$
\Omega \mathbf{j} \times \epsilon_{\mathbf{a c}}=\left\{\begin{array}{c}
\Omega(m \cos \theta-v \sin \theta) \\
0 \\
-\Omega(m \cos \theta-v \sin \theta)
\end{array}\right\} .
$$

The aforementioned manipulations result in the final equation for the absolute velocity of the aerodynamic centre in the coordinate system $\langle\mathbf{i}, \mathbf{j}, \mathbf{k}\rangle$, given as

$$
\left\{\begin{array}{c}
\dot{X} \\
\dot{Y} \\
\dot{Z}
\end{array}\right\}=\left\{\begin{array}{c}
(\dot{m}-\Omega v) \sin \theta-(\dot{v}-\Omega(m+r)) \cos \theta \\
\dot{n} \\
(\dot{m}-\Omega m) \cos \theta-(\dot{v}-\Omega(v-r)) \sin \theta
\end{array}\right\} .
$$




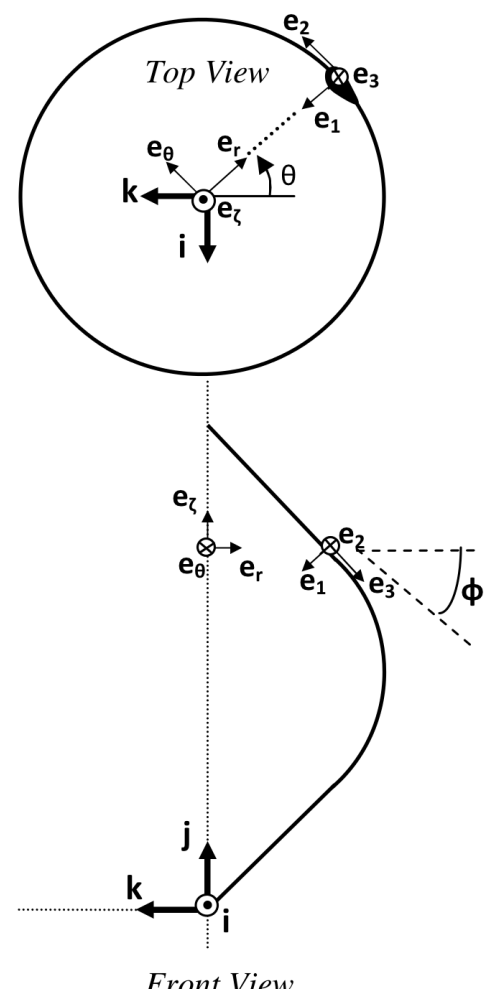

(a)

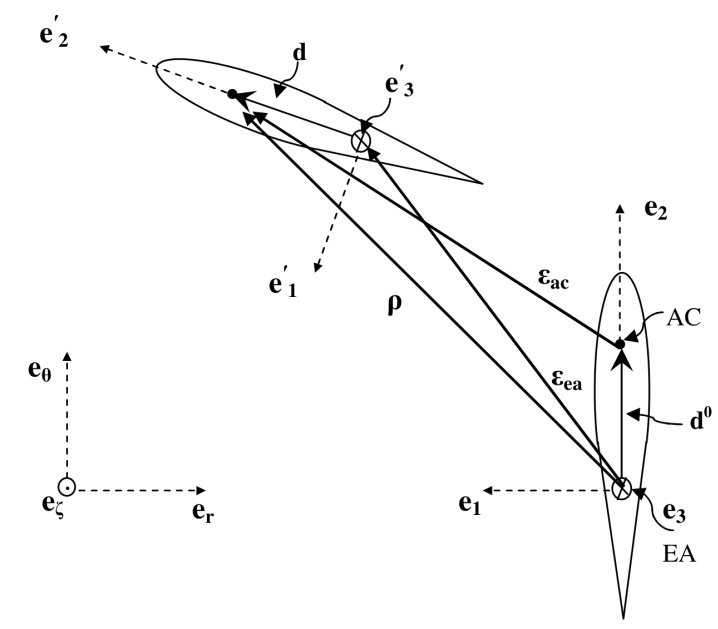

(b)

Figure A.1: (a) Coordinate systems (b) Blade section displacement 


\section{Bibliography}

[1] Thomas D Ashwill, Herbert J Sutherland, and Dale E Berg. A retrospective of VAWT technology. Technical report, Sandia National Laboratories, 2012.

[2] Walter Musial and Bonnie Ram. Large-scale offshore wind power in the United States: Assessment of opportunities and barriers. Technical report, National Renewable Energy Laboratory (NREL), Golden, CO., 2010.

[3] Ion Paraschivoiu. Wind turbine design: with emphasis on Darrieus concept. Presses inter Polytechnique, 2002.

[4] Ben F Blackwell, WN Sullivan, RC Reuter, and JF Banas. Engineering development status of the Darrieus Wind Turbine. Journal of Energy, 1(1):50-64, 1977.

[5] Paul S Veers. An Approach to the Fatigue Analysis of Vertical Axis Wind Turbine Blades. Technical report, Sandia National Labs., Albuquerque, NM (USA), 1981.

[6] RJ Templin. Aerodynamic performance theory for the NRC vertical axis wind turbine, NRC of Canada TR. Technical report, LTR-LA-160, 1974.

[7] James H Strickland. Darrieus turbine: a performance prediction model using multiple streamtubes. Technical report, Sandia Labs., Albuquerque, N. Mex.(USA), 1975.

[8] Ion Paraschivoiu. Double-multiple streamtube model for Darrieus in turbines. In Wind Turbine Dynamics, volume 1, pages 19-25, 1981.

[9] James H Strickland, BT Webster, and T Nguyen. Technical report, Sandia Labs., Albuquerque, N. Mex.(USA), 1979.

[10] RE Wilson and SN Walker. Fixed-wake analysis of the Darrieus rotor. Technical report, Sandia National Labs., Albuquerque, NM (USA); Oregon State Univ., Corvallis (USA). Dept. of Mechanical Engineering, 1981.

[11] James H Strickland, T Smith, and K Sun. Technical report, Sandia Labs., Albuquerque, N. Mex.(USA), 1981. 
[12] Donald W Lobitz. VAWT Rotor Structural Dynamics analysis methods. In Proceeding of the Vertical Axis Wind Turbine (VAWT) Design Technology Semina for Industry, pages 156-175, Albuquerque, New Mexico, April 1980.

[13] Robert Allan Watson. Comparison with strain gage data of centrifugal stresses predicted by finite element analysis on the DOE/Sandia 17-m Darrieus turbine. Technical report, Sandia Labs., Albuquerque, NM (USA), 1980.

[14] Thomas G Carne, Donald W Lobitz, AR Nord, and Robert A Watson. Finite element analysis and modal testing of a rotating wind turbine. Technical report, Sandia National Labs., Albuquerque, NM (USA), 1982.

[15] Donald W Lobitz and William N Sullivan. Comparison of finite element predictions and experimental data for the forced response of the DOE $100 \mathrm{~kW}$ vertical axis wind turbine. Technical report, Sandia National Labs., Albuquerque, NM (USA), 1984.

[16] Fred Nitzsche. Aeroelastic analysis of a Darrieus type wind turbine blade with troposkien geometry. $\mathrm{PhD}$ thesis, University of Stanford, California, US, 1983.

[17] David Popelka. Aeroelastic stability analysis of a Darrieus wind turbine. Technical report, Sandia National Labs., Albuquerque, NM (USA), 1982.

[18] Amin Fereidooni, Fred Nitzsche, and Edgar A Matida. Structural dynamic analysis of a troposkien shape vertical axis wind turbine using mixed finite element formulation. In Proceeding of the International Forum on Aeroelasticity and Structural Dynamics, Bristol, UK, June 2013.

[19] BF Blackwell and GE Reis. Blade shape for a troposkien type of vertical-axis wind turbine. Technical report, Sandia Labs., Albuquerque, NM (USA), 1977.

[20] S Nair and G Hegemier. Effect of initial stresses on the small deformations of a composite rod. AIAA Journal, 16(3):212-217, 1978.

[21] OC Zienkiewicz, RL Taylor, and JZ Zhu. The finite element method: its basis and fundamentals. 2005.

[22] Ivo Babuška. Error-bounds for finite element method. Numerische Mathematik, 16(4):322-333, 1971.

[23] Ivo Babuška. The finite element method with Lagrangian multipliers. Numerische Mathematik, 20(3):179-192, 1973.

[24] Franco Brezzi. On the existence, uniqueness and approximation of saddle-point problems arising from Lagrangian multipliers. ESAIM: Mathematical Modelling and $\mathrm{Nu}$ merical Analysis-Modélisation Mathématique et Analyse Numérique, 8(R2):129-151, 1974. 
[25] Robert E Sheldahl and Paul C Klimas. Aerodynamic characteristics of seven symmetrical airfoil sections through 180-degree angle of attack for use in aerodynamic analysis of vertical axis wind turbines. Technical report, Sandia National Labs., Albuquerque, NM (USA), 1981.

[26] Mark H Worstell. Aerodynamic Performance of the DOE/Sandia 17-m-Diameter Vertical-Axis Wind Turbine. Journal of Energy, 5(1):39-42, 1981.

[27] Gerald M McNerney. Accelerometer measurements of aerodynamic torque on the DOE/Sandia 17-m Vertical Axis Wind Turbine. Technical report, Sandia National Labs., Albuquerque, NM (USA), 1981.

[28] DE Berg. Recent improvements to the VDART3 VAWT code. Technical report, Sandia National Labs., Albuquerque, NM (USA), 1983. 